\title{
Palladium Complexes of a Phosphorus Ylide With Two Stabilizing Groups: Synthesis, Structure and DFT Study of the Bonding Modes.
}

Larry R. Falvello, ${ }^{\mathrm{a}}$ Juan Carlos Ginés, ${ }^{\mathrm{a}}$ Jorge J. Carbó, ${ }^{\mathrm{b}}$ Agustí Lledós*, ${ }^{\mathrm{c}}$ Rafael Navarro, ${ }^{\text {a }}$ Tatiana Soler ${ }^{\mathrm{d}}$ and Esteban P.Urriolabeitia*.

[a] Departamento de Compuestos Organometálicos, Instituto de Ciencia de Materiales de Aragón, Universidad de Zaragoza-C.S.I.C., 50009 Zaragoza (Spain). E-mail: esteban@unizar.es

[b] Departament de Química - Física i Inorgánica, Universitat Rovira i Virgili, 43007 Tarragona (Spain)

[c] Departament de Química, Edifici C.n., Universitat Autònoma de Barcelona, 08193 Bellaterra, Barcelona, Spain. E-mail: agusti@klingon.uab.es

[d] Servicio Técnicos de Investigación, Facultad de Ciencias Fase II, 03690 San Vicente de Raspeig, Alicante, Spain.

Dr. Esteban P. Urriolabeitia (corresponding author): Universidad de Zaragoza-C.S.I.C., Plaza de San Francisco s/n, E-50009 Zaragoza (Spain). Fax: (+34) 976761187. E-mail: esteban@unizar.es

\section{Supporting Information of IC060706F REVISED VERSION}


Table S1. Crystal data and structure refinement for L1.

Empirical formula

Formula weight

Temperature

Wavelength

Crystal system

Space group

Unit cell dimensions

Volume

Z

Density (calculated)

Absorption coefficient

$\mathrm{F}(000)$

Crystal size

Theta range for data collection

Index ranges

Reflections collected

Independent reflections

Completeness to theta $=25.05^{\circ}$

Absorption correction

Max. and min. transmission

Refinement method

Data / restraints / parameters

Goodness-of-fit on $\mathrm{F}^{2}$

Final $\mathrm{R}$ indices [I $>2 \operatorname{sigma}(\mathrm{I})]$

$\mathrm{R}$ indices (all data)

Largest diff. peak and hole

\section{C30 H26 N O4 P}

495.49

294(2) K

$0.71073 \AA$

Monoclinic

$\mathrm{P} 2(1) / \mathrm{n}$

$$
\begin{array}{ll}
\mathrm{a}=11.194(5) \AA & \alpha=90^{\circ} . \\
\mathrm{b}=13.585(6) \AA & \beta=90.853(9)^{\circ} . \\
\mathrm{c}=17.625(8) \AA & \gamma=90^{\circ} .
\end{array}
$$

2680(2) $\AA^{3}$

4

$$
1.228 \mathrm{Mg} / \mathrm{m}^{3}
$$

$0.137 \mathrm{~mm}^{-1}$

1040

$0.16 \times 0.12 \times 0.03 \mathrm{~mm}^{3}$

1.89 to $25.05^{\circ}$.

$-13<=\mathrm{h}<=13,-16<=\mathrm{k}<=16,-20<=\mathrm{l}<=20$

18490

$4733[\mathrm{R}($ int $)=0.1204]$

$99.8 \%$

None

0.9958 and 0.9783

Full-matrix least-squares on $\mathrm{F}^{2}$

4733 / 0 / 327

0.966

$\mathrm{R} 1=0.0656, \mathrm{wR} 2=0.1166$

$\mathrm{R} 1=0.1666, \mathrm{wR} 2=0.1506$

0.196 and -0.206 e. $\AA^{-3}$ 
Table S2. Atomic coordinates ( $\left.\times 10^{4}\right)$ and equivalent isotropic displacement parameters $\left(\AA^{2} \times 10^{3}\right)$ for L1. $U(e q)$ is defined as one third of the trace of the orthogonalized $\mathrm{U}^{\mathrm{ij}}$ tensor.

\begin{tabular}{|c|c|c|c|c|}
\hline & $\mathrm{x}$ & $\mathrm{y}$ & $\mathrm{z}$ & $\mathrm{U}(\mathrm{eq})$ \\
\hline $\mathrm{P}(1)$ & $9755(1)$ & $3599(1)$ & $8373(1)$ & $43(1)$ \\
\hline $\mathrm{O}(1)$ & $9403(3)$ & $581(2)$ & $6878(2)$ & $69(1)$ \\
\hline $\mathrm{N}(1)$ & $10006(3)$ & $1519(2)$ & $8412(2)$ & $54(1)$ \\
\hline $\mathrm{C}(1)$ & $9600(5)$ & $-62(4)$ & $6243(3)$ & $110(2)$ \\
\hline $\mathrm{O}(2)$ & $11270(3)$ & $1143(2)$ & $6791(2)$ & $67(1)$ \\
\hline $\mathrm{C}(2)$ & $10298(4)$ & $1171(3)$ & $7064(2)$ & $49(1)$ \\
\hline $\mathrm{O}(3)$ & $9227(4)$ & $4108(3)$ & $6723(2)$ & $105(1)$ \\
\hline $\mathrm{C}(3)$ & $9976(3)$ & $1833(3)$ & $7723(2)$ & $45(1)$ \\
\hline $\mathrm{O}(4)$ & $9436(3)$ & $2618(2)$ & $6255(2)$ & $78(1)$ \\
\hline $\mathrm{C}(4)$ & $9690(3)$ & $2850(3)$ & $7570(2)$ & $42(1)$ \\
\hline $\mathrm{C}(5)$ & $9454(4)$ & $3150(4)$ & $6808(3)$ & $56(1)$ \\
\hline $\mathrm{C}(6)$ & $9025(6)$ & $4474(4)$ & $5965(3)$ & $142(3)$ \\
\hline$C(7)$ & $10411(5)$ & $553(4)$ & $8601(3)$ & $65(1)$ \\
\hline $\mathrm{C}(8)$ & $11552(6)$ & 261(4) & $8508(3)$ & $101(2)$ \\
\hline $\mathrm{C}(9)$ & $11925(8)$ & $-658(6)$ & $8762(4)$ & $142(3)$ \\
\hline$C(10)$ & $11152(11)$ & $-1282(6)$ & $9100(5)$ & $156(5)$ \\
\hline $\mathrm{C}(11)$ & $10023(10)$ & $-984(5)$ & $9203(5)$ & $170(4)$ \\
\hline $\mathrm{C}(12)$ & $9643(6)$ & $-71(4)$ & $8957(4)$ & $120(2)$ \\
\hline $\mathrm{C}(13)$ & $8534(3)$ & $3419(3)$ & $9017(2)$ & $42(1)$ \\
\hline $\mathrm{C}(14)$ & $7729(4)$ & $2655(3)$ & $8905(2)$ & $55(1)$ \\
\hline$C(15)$ & $6811(4)$ & $2523(3)$ & $9416(3)$ & $65(1)$ \\
\hline$C(16)$ & $6705(4)$ & $3120(4)$ & $10037(3)$ & $63(1)$ \\
\hline $\mathrm{C}(17)$ & $7479(4)$ & $3889(3)$ & $10143(2)$ & $58(1)$ \\
\hline $\mathrm{C}(18)$ & $8385(4)$ & $4039(3)$ & $9634(2)$ & $53(1)$ \\
\hline $\mathrm{C}(19)$ & $11136(4)$ & $3352(3)$ & $8881(2)$ & $43(1)$ \\
\hline $\mathrm{C}(20)$ & $12142(4)$ & $3172(3)$ & $8457(2)$ & $60(1)$ \\
\hline $\mathrm{C}(21)$ & $13220(4)$ & $2955(3)$ & $8816(3)$ & $68(1)$ \\
\hline $\mathrm{C}(22)$ & $13292(4)$ & 2914(3) & $9587(3)$ & $69(1)$ \\
\hline $\mathrm{C}(23)$ & $12315(4)$ & $3097(3)$ & 10011(3) & $67(1)$ \\
\hline $\mathrm{C}(24)$ & $11233(4)$ & $3309(3)$ & $9663(2)$ & $54(1)$ \\
\hline$C(25)$ & $9720(4)$ & $4900(3)$ & $8161(2)$ & $43(1)$ \\
\hline$C(26)$ & $10759(4)$ & $5441(3)$ & $8101(2)$ & $57(1)$ \\
\hline $\mathrm{C}(27)$ & $10711(5)$ & $6439(4)$ & $7950(3)$ & $78(2)$ \\
\hline $\mathrm{C}(28)$ & $9634(5)$ & $6897(3)$ & $7855(3)$ & $76(2)$ \\
\hline $\mathrm{C}(29)$ & $8599(4)$ & $6377(3)$ & $7912(2)$ & $65(1)$ \\
\hline$C(30)$ & $8644(4)$ & $5377(3)$ & $8062(2)$ & $52(1)$ \\
\hline
\end{tabular}


Table S3. Bond lengths $[\AA ̊]$ and angles $\left[{ }^{\circ}\right]$ for $\mathrm{L} 1$.

\begin{tabular}{|c|c|}
\hline $\mathrm{P}(1)-\mathrm{C}(4)$ & $1.745(4)$ \\
\hline$P(1)-C(13)$ & $1.805(4)$ \\
\hline$P(1)-C(19)$ & $1.806(4)$ \\
\hline$P(1)-C(25)$ & $1.807(4)$ \\
\hline $\mathrm{O}(1)-\mathrm{C}(2)$ & $1.321(5)$ \\
\hline $\mathrm{O}(1)-\mathrm{C}(1)$ & $1.439(5)$ \\
\hline $\mathrm{N}(1)-\mathrm{C}(3)$ & $1.287(4)$ \\
\hline $\mathrm{N}(1)-\mathrm{C}(7)$ & $1.426(5)$ \\
\hline $\mathrm{O}(2)-\mathrm{C}(2)$ & $1.196(4)$ \\
\hline$C(2)-C(3)$ & $1.516(5)$ \\
\hline $\mathrm{O}(3)-\mathrm{C}(5)$ & $1.334(5)$ \\
\hline $\mathrm{O}(3)-\mathrm{C}(6)$ & $1.440(5)$ \\
\hline$C(3)-C(4)$ & $1.442(5)$ \\
\hline $\mathrm{O}(4)-\mathrm{C}(5)$ & $1.212(5)$ \\
\hline$C(4)-C(5)$ & $1.424(5)$ \\
\hline$C(7)-C(8)$ & $1.349(6)$ \\
\hline$C(7)-C(12)$ & $1.367(6)$ \\
\hline $\mathrm{C}(8)-\mathrm{C}(9)$ & $1.389(8)$ \\
\hline $\mathrm{C}(9)-\mathrm{C}(10)$ & $1.355(11)$ \\
\hline$C(10)-C(11)$ & $1.342(11)$ \\
\hline $\mathrm{C}(11)-\mathrm{C}(12)$ & $1.379(8)$ \\
\hline $\mathrm{C}(13)-\mathrm{C}(14)$ & $1.387(5)$ \\
\hline $\mathrm{C}(13)-\mathrm{C}(18)$ & $1.387(5)$ \\
\hline $\mathrm{C}(14)-\mathrm{C}(15)$ & $1.388(6)$ \\
\hline $\mathrm{C}(15)-\mathrm{C}(16)$ & $1.369(6)$ \\
\hline$C(16)-C(17)$ & $1.368(5)$ \\
\hline $\mathrm{C}(17)-\mathrm{C}(18)$ & $1.380(5)$ \\
\hline $\mathrm{C}(19)-\mathrm{C}(24)$ & $1.382(5)$ \\
\hline $\mathrm{C}(19)-\mathrm{C}(20)$ & $1.383(5)$ \\
\hline $\mathrm{C}(20)-\mathrm{C}(21)$ & $1.386(6)$ \\
\hline $\mathrm{C}(21)-\mathrm{C}(22)$ & $1.361(6)$ \\
\hline $\mathrm{C}(22)-\mathrm{C}(23)$ & $1.357(6)$ \\
\hline $\mathrm{C}(23)-\mathrm{C}(24)$ & $1.379(5)$ \\
\hline $\mathrm{C}(25)-\mathrm{C}(30)$ & $1.376(5)$ \\
\hline $\mathrm{C}(25)-\mathrm{C}(26)$ & $1.381(5)$ \\
\hline $\mathrm{C}(26)-\mathrm{C}(27)$ & $1.383(5)$ \\
\hline $\mathrm{C}(27)-\mathrm{C}(28)$ & $1.365(6)$ \\
\hline $\mathrm{C}(28)-\mathrm{C}(29)$ & $1.362(6)$ \\
\hline $\mathrm{C}(29)-\mathrm{C}(30)$ & $1.385(5)$ \\
\hline $\mathrm{C}(4)-\mathrm{P}(1)-\mathrm{C}(13)$ & $114.13(18)$ \\
\hline $\mathrm{C}(4)-\mathrm{P}(1)-\mathrm{C}(19)$ & $108.60(18)$ \\
\hline $\mathrm{C}(13)-\mathrm{P}(1)-\mathrm{C}(19)$ & $108.30(19)$ \\
\hline $\mathrm{C}(4)-\mathrm{P}(1)-\mathrm{C}(25)$ & $113.70(18)$ \\
\hline $\mathrm{C}(13)-\mathrm{P}(1)-\mathrm{C}(25)$ & $104.34(18)$ \\
\hline $\mathrm{C}(19)-\mathrm{P}(1)-\mathrm{C}(25)$ & $107.45(18)$ \\
\hline $\mathrm{C}(2)-\mathrm{O}(1)-\mathrm{C}(1)$ & $115.9(3)$ \\
\hline $\mathrm{C}(3)-\mathrm{N}(1)-\mathrm{C}(7)$ & $122.0(4)$ \\
\hline $\mathrm{O}(2)-\mathrm{C}(2)-\mathrm{O}(1)$ & $124.9(4)$ \\
\hline $\mathrm{O}(2)-\mathrm{C}(2)-\mathrm{C}(3)$ & $123.7(4)$ \\
\hline $\mathrm{O}(1)-\mathrm{C}(2)-\mathrm{C}(3)$ & 111.2(4) \\
\hline $\mathrm{C}(5)-\mathrm{O}(3)-\mathrm{C}(6)$ & $117.9(4)$ \\
\hline
\end{tabular}




$\begin{array}{ll}\text { N(1)-C(3)-C(4) } & 119.8(4) \\ \text { N(1)-C(3)-C(2) } & 121.5(4) \\ \mathrm{C}(4)-\mathrm{C}(3)-\mathrm{C}(2) & 118.7(4) \\ \mathrm{C}(5)-\mathrm{C}(4)-\mathrm{C}(3) & 119.2(4) \\ \mathrm{C}(5)-\mathrm{C}(4)-\mathrm{P}(1) & 127.2(3) \\ \mathrm{C}(3)-\mathrm{C}(4)-\mathrm{P}(1) & 113.6(3) \\ \mathrm{O}(4)-\mathrm{C}(5)-\mathrm{O}(3) & 119.4(4) \\ \mathrm{O}(4)-\mathrm{C}(5)-\mathrm{C}(4) & 126.0(4) \\ \mathrm{O}(3)-\mathrm{C}(5)-\mathrm{C}(4) & 114.6(4) \\ \mathrm{C}(8)-\mathrm{C}(7)-\mathrm{C}(12) & 118.4(5) \\ \mathrm{C}(8)-\mathrm{C}(7)-\mathrm{N}(1) & 122.7(5) \\ \mathrm{C}(12)-\mathrm{C}(7)-\mathrm{N}(1) & 118.5(5) \\ \mathrm{C}(7)-\mathrm{C}(8)-\mathrm{C}(9) & 120.4(7) \\ \mathrm{C}(10)-\mathrm{C}(9)-\mathrm{C}(8) & 120.9(9) \\ \mathrm{C}(11)-\mathrm{C}(10)-\mathrm{C}(9) & 118.6(8) \\ \mathrm{C}(10)-\mathrm{C}(11)-\mathrm{C}(12) & 121.1(9) \\ \mathrm{C}(7)-\mathrm{C}(12)-\mathrm{C}(11) & 120.6(7) \\ \mathrm{C}(14)-\mathrm{C}(13)-\mathrm{C}(18) & 118.6(4) \\ \mathrm{C}(14)-\mathrm{C}(13)-\mathrm{P}(1) & 120.6(3) \\ \mathrm{C}(18)-\mathrm{C}(13)-\mathrm{P}(1) & 120.8(3) \\ \mathrm{C}(13)-\mathrm{C}(14)-\mathrm{C}(15) & 119.4(4) \\ \mathrm{C}(16)-\mathrm{C}(15)-\mathrm{C}(14) & 121.0(4) \\ \mathrm{C}(17)-\mathrm{C}(16)-\mathrm{C}(15) & 120.0(4) \\ \mathrm{C}(16)-\mathrm{C}(17)-\mathrm{C}(18) & 119.6(4) \\ \mathrm{C}(17)-\mathrm{C}(18)-\mathrm{C}(13) & 121.3(4) \\ \mathrm{C}(24)-\mathrm{C}(19)-\mathrm{C}(20) & 118.6(4) \\ \mathrm{C}(24)-\mathrm{C}(19)-\mathrm{P}(1) & 123.8(3) \\ \mathrm{C}(20)-\mathrm{C}(19)-\mathrm{P}(1) & 117.6(3) \\ \mathrm{C}(19)-\mathrm{C}(20)-\mathrm{C}(21) & 120.1(4) \\ \mathrm{C}(22)-\mathrm{C}(21)-\mathrm{C}(20) & 120.2(4) \\ \mathrm{C}(23)-\mathrm{C}(22)-\mathrm{C}(21) & 120.4(5) \\ \mathrm{C}(22)-\mathrm{C}(23)-\mathrm{C}(24) & 120.2(4) \\ \mathrm{C}(23)-\mathrm{C}(24)-\mathrm{C}(19) & 120.5(4) \\ \mathrm{C}(30)-\mathrm{C}(25)-\mathrm{C}(26) & 118.4(4) \\ \mathrm{C}(30)-\mathrm{C}(25)-\mathrm{P}(1) & 120.2(3) \\ \mathrm{C}(26)-\mathrm{C}(25)-\mathrm{P}(1) & 121.3(3) \\ \mathrm{C}(25)-\mathrm{C}(26)-\mathrm{C}(27) & 120.4(4) \\ \mathrm{C}(28)-\mathrm{C}(27)-\mathrm{C}(26) & 120.1(5) \\ \mathrm{C}(29)-\mathrm{C}(28)-\mathrm{C}(27) & 120.4(4) \\ \mathrm{C}(28)-\mathrm{C}(29)-\mathrm{C}(30) & 119.6(4) \\ \mathrm{C}(25)-\mathrm{C}(30)-\mathrm{C}(29) & 121.1(4) \\ & \end{array}$


Table S4. Anisotropic displacement parameters $\left(\AA^{2} \times 10^{3}\right)$ for L1. The anisotropic displacement factor exponent takes the form: $-2 \pi^{2}\left[h^{2} a^{* 2} U^{11}+\ldots+2 h k a^{*} b^{*} U^{12}\right]$

\begin{tabular}{|c|c|c|c|c|c|c|}
\hline & $\mathrm{U}^{11}$ & $\mathrm{U}^{22}$ & $\mathrm{U}^{33}$ & $\mathrm{U}^{23}$ & $\mathrm{U}^{13}$ & $\mathrm{U}^{12}$ \\
\hline $\mathrm{P}(1)$ & $43(1)$ & $46(1)$ & $39(1)$ & $-4(1)$ & $2(1)$ & $-1(1)$ \\
\hline $\mathrm{O}(1)$ & $53(2)$ & $72(2)$ & $81(2)$ & $-34(2)$ & $6(2)$ & $-15(2$ \\
\hline $\mathrm{N}(1)$ & $67(3)$ & $49(2)$ & $46(2)$ & $-2(2)$ & $5(2)$ & $1(2)$ \\
\hline $\mathrm{C}(1)$ & $105(5)$ & $106(5)$ & $118(5)$ & $-77(4)$ & $12(4)$ & $-16(4$ \\
\hline $\mathrm{O}(2)$ & $47(2)$ & $92(2)$ & $64(2)$ & $-16(2)$ & $10(2)$ & $7(2)$ \\
\hline $\mathrm{C}(2)$ & $47(3)$ & $54(3)$ & $44(3)$ & $-4(2)$ & $-1(2)$ & $4(2)$ \\
\hline $\mathrm{O}(3)$ & 201(4) & $64(2)$ & $50(2)$ & $4(2)$ & $-25(2)$ & $29(2)$ \\
\hline $\mathrm{C}(3)$ & $34(3)$ & $56(3)$ & $46(3)$ & $-6(2)$ & $1(2)$ & $-7(2)$ \\
\hline $\mathrm{O}(4)$ & $107(3)$ & $86(2)$ & $42(2)$ & $-15(2)$ & $-18(2)$ & $24(2)$ \\
\hline $\mathrm{C}(4)$ & $47(3)$ & $48(3)$ & $32(2)$ & $-3(2)$ & $1(2)$ & $0(2)$ \\
\hline $\mathrm{C}(5)$ & $57(3)$ & $62(3)$ & $50(3)$ & $-6(3)$ & $-3(2)$ & $7(3)$ \\
\hline $\mathrm{C}(6)$ & $264(9)$ & $90(4)$ & $70(4)$ & $25(3)$ & $-34(5)$ & $47(5)$ \\
\hline $\mathrm{C}(7)$ & $80(4)$ & $56(3)$ & $58(3)$ & $1(3)$ & $-4(3)$ & $5(3)$ \\
\hline $\mathrm{C}(8)$ & $105(5)$ & $84(4)$ & $115(5)$ & $12(4)$ & $-16(4)$ & $28(4)$ \\
\hline $\mathrm{C}(9)$ & $165(9)$ & $101(6)$ & $158(8)$ & $-10(5)$ & $-62(6)$ & $51(6)$ \\
\hline $\mathrm{C}(10)$ & $252(14)$ & $64(5)$ & $150(8)$ & $-2(5)$ & $-68(8)$ & $50(7)$ \\
\hline $\mathrm{C}(11)$ & $257(12)$ & $59(5)$ & 196(9) & $55(5)$ & $8(8)$ & $-7(6)$ \\
\hline $\mathrm{C}(12)$ & $116(6)$ & $75(4)$ & $169(7)$ & $34(4)$ & $32(5)$ & $-4(4)$ \\
\hline $\mathrm{C}(13)$ & $44(3)$ & $42(2)$ & $39(2)$ & $-5(2)$ & $2(2)$ & $0(2)$ \\
\hline $\mathrm{C}(14)$ & $54(3)$ & $55(3)$ & $57(3)$ & $-7(2)$ & $2(2)$ & $-1(2)$ \\
\hline$C(15)$ & $48(3)$ & $65(3)$ & $82(4)$ & $5(3)$ & $8(3)$ & $-12(3$ \\
\hline$C(16)$ & $58(3)$ & $77(4)$ & $55(3)$ & $12(3)$ & $13(3)$ & $3(3)$ \\
\hline $\mathrm{C}(17)$ & $59(3)$ & $72(3)$ & 41(3) & $-4(2)$ & $7(2)$ & $7(3)$ \\
\hline $\mathrm{C}(18)$ & $51(3)$ & $60(3)$ & $48(3)$ & $-6(2)$ & $2(2)$ & $-3(2)$ \\
\hline $\mathrm{C}(19)$ & $45(3)$ & $43(3)$ & $43(3)$ & $-6(2)$ & $-1(2)$ & $1(2)$ \\
\hline$C(20)$ & $54(3)$ & $80(3)$ & $47(3)$ & $-1(2)$ & $-6(3)$ & $3(3)$ \\
\hline $\mathrm{C}(21)$ & $45(3)$ & $95(4)$ & $66(4)$ & $-8(3)$ & $4(3)$ & $5(3)$ \\
\hline $\mathrm{C}(22)$ & $52(3)$ & $86(4)$ & $67(4)$ & $-4(3)$ & $-15(3)$ & $7(3)$ \\
\hline $\mathrm{C}(23)$ & $63(4)$ & $85(4)$ & $51(3)$ & $5(3)$ & $-12(3)$ & $6(3)$ \\
\hline$C(24)$ & $56(3)$ & $59(3)$ & $47(3)$ & $1(2)$ & $-1(2)$ & $3(2)$ \\
\hline$C(25)$ & $42(3)$ & $43(2)$ & $44(3)$ & $0(2)$ & $5(2)$ & $0(2)$ \\
\hline$C(26)$ & $47(3)$ & $52(3)$ & 71(3) & $7(2)$ & $5(2)$ & $-1(2)$ \\
\hline $\mathrm{C}(27)$ & $66(4)$ & $55(3)$ & $113(4)$ & $20(3)$ & $7(3)$ & $-13(3$ \\
\hline $\mathrm{C}(28)$ & $83(4)$ & $49(3)$ & $95(4)$ & $12(3)$ & $7(3)$ & $0(3)$ \\
\hline $\mathrm{C}(29)$ & $67(4)$ & $61(3)$ & $67(3)$ & $9(3)$ & $7(3)$ & $17(3)$ \\
\hline $\mathrm{C}(30)$ & $48(3)$ & $53(3)$ & $57(3)$ & $-2(2)$ & $4(2)$ & $1(2)$ \\
\hline
\end{tabular}


Table S5. Hydrogen coordinates $\left(\times 10^{4}\right)$ and isotropic displacement parameters $\left(\AA^{2} \times 10^{3}\right)$ for L1.

\begin{tabular}{lrrrr}
\hline & $\mathrm{x}$ & $\mathrm{y}$ & $\mathrm{z}$ & $\mathrm{U}(\mathrm{eq})$ \\
\hline & & & & \\
$\mathrm{H}(1 \mathrm{~A})$ & 9780 & 324 & 5803 & 164 \\
$\mathrm{H}(1 \mathrm{~B})$ & 8893 & -445 & 6146 & 164 \\
$\mathrm{H}(1 \mathrm{C})$ & 10257 & -493 & 6358 & 164 \\
$\mathrm{H}(6 \mathrm{~A})$ & 9717 & 4349 & 5665 & 213 \\
$\mathrm{H}(6 \mathrm{~B})$ & 8878 & 5170 & 5984 & 213 \\
$\mathrm{H}(6 \mathrm{C})$ & 8345 & 4148 & 5742 & 213 \\
$\mathrm{H}(8)$ & 12092 & 678 & 8273 & 121 \\
$\mathrm{H}(9)$ & 12715 & -849 & 8700 & 171 \\
$\mathrm{H}(10)$ & 11399 & -1904 & 9258 & 187 \\
$\mathrm{H}(11)$ & 9488 & -1400 & 9443 & 205 \\
$\mathrm{H}(12)$ & 8857 & 122 & 9035 & 144 \\
$\mathrm{H}(14)$ & 7804 & 2235 & 8492 & 67 \\
$\mathrm{H}(15)$ & 6260 & 2020 & 9335 & 78 \\
$\mathrm{H}(16)$ & 6106 & 3004 & 10386 & 76 \\
$\mathrm{H}(17)$ & 7395 & 4307 & 10557 & 69 \\
$\mathrm{H}(18)$ & 8905 & 4566 & 9705 & 64 \\
$\mathrm{H}(20)$ & 12096 & 3196 & 7930 & 72 \\
$\mathrm{H}(21)$ & 13896 & 2837 & 8529 & 82 \\
$\mathrm{H}(22)$ & 14015 & 2759 & 9824 & 82 \\
$\mathrm{H}(23)$ & 12374 & 3080 & 10537 & 80 \\
$\mathrm{H}(24)$ & 10565 & 3424 & 9957 & 65 \\
$\mathrm{H}(26)$ & 11495 & 5131 & 8162 & 68 \\
$\mathrm{H}(27)$ & 11415 & 6799 & 7914 & 94 \\
$\mathrm{H}(28)$ & 9607 & 7568 & 7751 & 91 \\
$\mathrm{H}(29)$ & 7866 & 6692 & 7851 & 78 \\
$\mathrm{H}(30)$ & 7937 & 5022 & 8097 & 63 \\
& & & \\
\hline
\end{tabular}


Table S6. Crystal data and structure refinement for 3.

Empirical formula

Formula weight

Temperature

Wavelength

Crystal system

Space group

Unit cell dimensions

Volume

Z

Density (calculated)

Absorption coefficient

$\mathrm{F}(000)$

Crystal size

Theta range for data collection

Index ranges

Reflections collected

Independent reflections

Completeness to theta $=30.00^{\circ}$

Absorption correction

Max. and min. transmission

Refinement method

Data / restraints / parameters

Goodness-of-fit on $\mathrm{F}^{2}$

Final $\mathrm{R}$ indices [I $>2 \operatorname{sigma}(\mathrm{I})]$

$\mathrm{R}$ indices (all data)

Largest diff. peak and hole
C40 H34 Cl N2 O8 P Pd

843.51

123(1) K

$0.7107 \AA$

Monoclinic

P 21/c

$\begin{array}{ll}\mathrm{a}=24.3083(5) \AA & \alpha=90^{\circ} . \\ \mathrm{b}=14.5144(2) \AA & \beta=115.874(3)^{\circ} . \\ \mathrm{c}=23.1589(4) \AA & \gamma=90^{\circ} .\end{array}$

$7351.8(2) \AA^{3}$

8

$1.524 \mathrm{Mg} / \mathrm{m}^{3}$

$0.678 \mathrm{~mm}^{-1}$

3440

$0.42 \times 0.08 \times 0.04 \mathrm{~mm}^{3}$

3.65 to $30.00^{\circ}$.

$-34<=\mathrm{h}<=34,-20<=\mathrm{k}<=20,-32<=1<=32$

146673

$21409[\mathrm{R}(\mathrm{int})=0.0886]$

$99.8 \%$

Numerical

0.96719 and 0.85189

Full-matrix least-squares on $\mathrm{F}^{2}$

21409 / 0 / 959

1.006

$\mathrm{R} 1=0.0376, \mathrm{wR} 2=0.0699$

$\mathrm{R} 1=0.1076, \mathrm{wR} 2=0.1005$

0.881 and -0.426 e. $\AA^{-3}$ 
Table S7. Atomic coordinates $\left(\times 10^{4}\right)$ and equivalent isotropic displacement parameters $\left(\AA^{2} \times 10^{3}\right)$ for 3. $U(e q)$ is defined as one third of the trace of the orthogonalized $U^{i j}$ tensor.

\begin{tabular}{|c|c|c|c|c|}
\hline & $\mathrm{X}$ & $\mathrm{y}$ & $\mathrm{z}$ & $\mathrm{U}(\mathrm{eq})$ \\
\hline $\operatorname{Pd}(1)$ & $9109(1)$ & $9026(1)$ & $7002(1)$ & $22(1)$ \\
\hline $\mathrm{C}(1)$ & $9333(2)$ & $9920(2)$ & $7744(2)$ & $25(1)$ \\
\hline$C(2)$ & $9431(2)$ & $10840(2)$ & $7526(2)$ & $22(1)$ \\
\hline$C(3)$ & $9468(2)$ & $11653(3)$ & $7833(2)$ & $27(1)$ \\
\hline $\mathrm{C}(4)$ & $9571(2)$ & $12497(3)$ & $7581(2)$ & $31(1)$ \\
\hline$C(5)$ & $9615(2)$ & $12519(3)$ & $7005(2)$ & $29(1)$ \\
\hline$C(6)$ & $9576(2)$ & $11688(2)$ & $6666(2)$ & $25(1)$ \\
\hline$C(7)$ & $9494(2)$ & $10866(2)$ & $6945(2)$ & $21(1)$ \\
\hline $\mathrm{C}(8)$ & $9604(2)$ & $11619(3)$ & $6080(2)$ & $31(1)$ \\
\hline $\mathrm{C}(9)$ & $9572(2)$ & 10784(3) & $5809(2)$ & $34(1)$ \\
\hline$C(10)$ & $9486(2)$ & 9995(3) & $6103(2)$ & $29(1)$ \\
\hline $\mathrm{N}(1)$ & $9435(1)$ & $10037(2)$ & $6647(1)$ & $23(1)$ \\
\hline $\mathrm{O}(1)$ & $8890(1)$ & $8278(2)$ & $6169(1)$ & $29(1)$ \\
\hline $\mathrm{C}(11)$ & $8624(2)$ & $7542(3)$ & $6023(2)$ & $23(1)$ \\
\hline $\mathrm{O}(2)$ & $8429(1)$ & $7254(2)$ & $5418(1)$ & $25(1)$ \\
\hline$C(12)$ & $8504(2)$ & $7884(2)$ & $4977(2)$ & $30(1)$ \\
\hline$C(13)$ & $8486(2)$ & $6917(2)$ & $6426(2)$ & $21(1)$ \\
\hline $\mathrm{P}(1)$ & $8136(1)$ & $5887(1)$ & $6020(1)$ & $23(1)$ \\
\hline$C(14)$ & $7456(2)$ & $6208(2)$ & $5310(2)$ & $23(1)$ \\
\hline$C(15)$ & $7072(2)$ & $6839(2)$ & $5384(2)$ & $29(1)$ \\
\hline$C(16)$ & $6584(2)$ & $7178(3)$ & $4857(2)$ & $35(1)$ \\
\hline$C(17)$ & $6489(2)$ & $6899(3)$ & $4248(2)$ & $35(1)$ \\
\hline $\mathrm{C}(18)$ & $6871(2)$ & $6251(3)$ & $4170(2)$ & $34(1)$ \\
\hline C(19) & $7342(2)$ & $5913(3)$ & $4698(2)$ & $29(1)$ \\
\hline$C(20)$ & $7919(2)$ & $5074(2)$ & $6468(2)$ & $24(1)$ \\
\hline $\mathrm{C}(21)$ & $8343(2)$ & $4424(3)$ & $6858(2)$ & $37(1)$ \\
\hline $\mathrm{C}(22)$ & $8163(2)$ & $3751(3)$ & $7161(2)$ & $43(1)$ \\
\hline$C(23)$ & $7570(2)$ & $3732(3)$ & $7092(2)$ & $36(1)$ \\
\hline $\mathrm{C}(24)$ & $7153(2)$ & $4379(3)$ & $6726(2)$ & $35(1)$ \\
\hline$C(25)$ & $7317(2)$ & $5046(3)$ & $6399(2)$ & $31(1)$ \\
\hline$C(26)$ & $8629(2)$ & $5229(3)$ & $5772(2)$ & $25(1)$ \\
\hline$C(27)$ & $9192(2)$ & $5564(3)$ & $5839(2)$ & $25(1)$ \\
\hline $\mathrm{C}(28)$ & $9535(2)$ & $5044(3)$ & $5610(2)$ & $30(1)$ \\
\hline$C(29)$ & $9324(2)$ & $4205(3)$ & $5318(2)$ & $28(1)$ \\
\hline$C(30)$ & $8776(2)$ & $3859(3)$ & $5268(2)$ & $31(1)$ \\
\hline $\mathrm{C}(31)$ & $8433(2)$ & $4358(3)$ & $5495(2)$ & $31(1)$ \\
\hline$C(32)$ & $8534(2)$ & $7209(2)$ & $7028(2)$ & $22(1)$ \\
\hline $\mathrm{C}(33)$ & $8359(2)$ & $6555(3)$ & $7438(2)$ & $28(1)$ \\
\hline $\mathrm{O}(3)$ & $7853(1)$ & $6513(2)$ & $7402(1)$ & $39(1)$ \\
\hline $\mathrm{O}(4)$ & $8849(1)$ & $6107(2)$ & $7844(1)$ & $42(1)$ \\
\hline$C(34)$ & $8768(2)$ & $5569(3)$ & $8321(2)$ & $73(2)$ \\
\hline $\mathrm{N}(2)$ & $8718(1)$ & $8008(2)$ & $7305(1)$ & $21(1)$ \\
\hline$C(35)$ & $8625(2)$ & $8234(2)$ & $7865(2)$ & $22(1)$ \\
\hline$C(36)$ & $9099(2)$ & $8183(3)$ & $8473(2)$ & $34(1)$ \\
\hline$C(37)$ & $9019(2)$ & 8491(3) & $8999(2)$ & $40(1)$ \\
\hline $\mathrm{C}(38)$ & $8463(2)$ & $8857(3)$ & $8907(2)$ & $39(1)$ \\
\hline C(39) & $7986(2)$ & $8897(3)$ & $8304(2)$ & $34(1)$ \\
\hline $\mathrm{C}(40)$ & $8067(2)$ & $8580(3)$ & $7780(2)$ & $29(1)$ \\
\hline $\operatorname{Pd}(2)$ & $6017(1)$ & $4009(1)$ & $3560(1)$ & $21(1)$ \\
\hline $\mathrm{C}(41)$ & $5936(2)$ & $4805(2)$ & $2819(2)$ & $24(1)$ \\
\hline $\mathrm{C}(42)$ & $5597(2)$ & $5665(2)$ & $2808(2)$ & $23(1)$ \\
\hline
\end{tabular}




\begin{tabular}{|c|c|c|c|c|}
\hline $\mathrm{C}(43)$ & $5515(2)$ & $6430(3)$ & $2423(2)$ & $26(1)$ \\
\hline C(44) & $5140(2)$ & 7169(2) & $2430(2)$ & $28(1)$ \\
\hline$C(45)$ & $4850(2)$ & $7170(2)$ & $2818(2)$ & $27(1)$ \\
\hline$C(46)$ & 4934(2) & 6423(2) & $3240(2)$ & $25(1)$ \\
\hline C(47) & $5327(2)$ & $5689(2)$ & $3240(2)$ & $22(1)$ \\
\hline $\mathrm{C}(48)$ & 4677(2) & 6343(3) & $3682(2)$ & $32(1)$ \\
\hline C(49) & $4828(2)$ & $5615(3)$ & $4096(2)$ & $35(1)$ \\
\hline $\mathrm{C}(50)$ & $5226(2)$ & 4922(3) & $4075(2)$ & $32(1)$ \\
\hline $\mathrm{N}(3)$ & 5463(1) & 4952(2) & $3659(1)$ & $24(1)$ \\
\hline $\mathrm{O}(5)$ & 6109(1) & $3286(2)$ & $4384(1)$ & $28(1)$ \\
\hline $\mathrm{C}(51)$ & 6204(2) & 2449(3) & $4466(2)$ & $23(1)$ \\
\hline $\mathrm{O}(6)$ & $6208(1)$ & 2034(2) & $4985(1)$ & $25(1)$ \\
\hline C(52) & 6082(2) & 2629(3) & $5418(2)$ & $33(1)$ \\
\hline C(53) & 6347(2) & 1817(2) & $4067(2)$ & $21(1)$ \\
\hline $\mathrm{P}(2)$ & 6318(1) & $636(1)$ & $4236(1)$ & $22(1)$ \\
\hline C(54) & $5582(2)$ & $263(2)$ & $4153(2)$ & $21(1)$ \\
\hline C(55) & $5470(2)$ & $-684(2)$ & $4116(2)$ & $26(1)$ \\
\hline C(56) & 4943(2) & $-1028(3)$ & $4106(2)$ & $29(1)$ \\
\hline C(57) & 4503(2) & $-434(3)$ & $4109(2)$ & $32(1)$ \\
\hline C(58) & 4589(2) & $510(3)$ & $4116(2)$ & $31(1)$ \\
\hline C(59) & $5134(2)$ & $856(2)$ & $4144(2)$ & $25(1)$ \\
\hline $\mathrm{C}(60)$ & 6857(2) & $362(2)$ & $5039(2)$ & $23(1)$ \\
\hline $\mathrm{C}(61)$ & 7399(2) & $851(3)$ & $5315(2)$ & $36(1)$ \\
\hline C(62) & 7817(2) & $663(3)$ & $5940(2)$ & $44(1)$ \\
\hline C(63) & 7694(2) & $-35(3)$ & $6283(2)$ & $39(1)$ \\
\hline C(64) & 7163(2) & $-511(3)$ & $6009(2)$ & $35(1)$ \\
\hline$C(65)$ & 6747(2) & $-318(3)$ & $5392(2)$ & $30(1)$ \\
\hline$C(66)$ & $6457(2)$ & $-85(2)$ & $3689(2)$ & $22(1)$ \\
\hline $\mathrm{C}(67)$ & 6028(2) & $-101(3)$ & $3054(2)$ & $27(1)$ \\
\hline $\mathrm{C}(68)$ & 6098(2) & $-671(3)$ & $2610(2)$ & $32(1)$ \\
\hline C(69) & 6597(2) & $-1233(3)$ & $2805(2)$ & $36(1)$ \\
\hline C(70) & $7026(2)$ & $-1232(3)$ & $3438(2)$ & $36(1)$ \\
\hline C(71) & 6954(2) & $-660(2)$ & $3884(2)$ & $29(1)$ \\
\hline C(72) & $6600(2)$ & 2163(2) & $3656(2)$ & $21(1)$ \\
\hline C(73) & 7011(2) & 1544(2) & $3478(2)$ & $27(1)$ \\
\hline $\mathrm{O}(7)$ & 6887(1) & 1307(2) & $2947(1)$ & $34(1)$ \\
\hline $\mathrm{O}(8)$ & 7518(1) & 1364(2) & $4002(1)$ & $33(1)$ \\
\hline C(74) & 7971(2) & $877(3)$ & $3880(2)$ & $50(1)$ \\
\hline $\mathrm{N}(4)$ & $6548(1)$ & 3019(2) & $3437(1)$ & $22(1)$ \\
\hline$C(75)$ & 6973(2) & $3283(2)$ & $3180(2)$ & $25(1)$ \\
\hline$C(76)$ & 7588(2) & $3417(2)$ & $3598(2)$ & $30(1)$ \\
\hline $\mathrm{C}(77)$ & $8008(2)$ & $3638(3)$ & $3363(2)$ & $36(1)$ \\
\hline C(78) & $7820(2)$ & 3751(3) & $2717(2)$ & $35(1)$ \\
\hline C(79) & 7202(2) & 3643(2) & $2298(2)$ & $30(1)$ \\
\hline $\mathrm{C}(80)$ & $6775(2)$ & $3409(2)$ & $2526(2)$ & $25(1)$ \\
\hline $\mathrm{Cl}(2)$ & 4193(1) & 3008(1) & $4078(1)$ & $35(1)$ \\
\hline $\mathrm{O}(13)$ & $3668(1)$ & 2453(2) & $3781(2)$ & $56(1)$ \\
\hline $\mathrm{O}(14)$ & 4563(2) & $2668(2)$ & $4708(1)$ & $65(1)$ \\
\hline $\mathrm{O}(15)$ & 4019(1) & 3932(2) & $4120(2)$ & $62(1)$ \\
\hline $\mathrm{O}(16)$ & $4546(1)$ & 2970(2) & $3717(1)$ & $45(1)$ \\
\hline $\mathrm{Cl}(1)$ & $9625(1)$ & 2026(1) & $4368(1)$ & $32(1)$ \\
\hline $\mathrm{O}(9)$ & $10067(1)$ & 1357(2) & $4763(2)$ & $52(1)$ \\
\hline $\mathrm{O}(10)$ & 9939(1) & 2862(2) & $4368(1)$ & $50(1)$ \\
\hline $\mathrm{O}(11)$ & 9303(2) & 1697(2) & $3726(1)$ & $56(1)$ \\
\hline $\mathrm{O}(12)$ & 9197(1) & 2211(2) & $4628(1)$ & $41(1)$ \\
\hline
\end{tabular}


Table S8. Bond lengths $[\AA]$ and angles $\left[^{\circ}\right]$ for 3 .

\begin{tabular}{|c|c|}
\hline$\overline{\mathrm{Pd}(1)-\mathrm{N}(1)}$ & $2.006(3)$ \\
\hline $\operatorname{Pd}(1)-C(1)$ & $2.028(3)$ \\
\hline $\operatorname{Pd}(1)-\mathrm{N}(2)$ & $2.041(3)$ \\
\hline $\mathrm{Pd}(1)-\mathrm{O}(1)$ & $2.070(2)$ \\
\hline$C(1)-C(2)$ & $1.483(5)$ \\
\hline $\mathrm{C}(1)-\mathrm{H}(1 \mathrm{~A})$ & 0.9700 \\
\hline $\mathrm{C}(1)-\mathrm{H}(1 \mathrm{~B})$ & 0.9700 \\
\hline $\mathrm{C}(2)-\mathrm{C}(3)$ & $1.361(5)$ \\
\hline$C(2)-C(7)$ & $1.420(4)$ \\
\hline$C(3)-C(4)$ & $1.425(5)$ \\
\hline $\mathrm{C}(3)-\mathrm{H}(3)$ & 0.9300 \\
\hline$C(4)-C(5)$ & $1.384(5)$ \\
\hline $\mathrm{C}(4)-\mathrm{H}(4)$ & 0.9300 \\
\hline$C(5)-C(6)$ & $1.420(5)$ \\
\hline $\mathrm{C}(5)-\mathrm{H}(5)$ & 0.9300 \\
\hline $\mathrm{C}(6)-\mathrm{C}(8)$ & $1.391(5)$ \\
\hline$C(6)-C(7)$ & $1.413(5)$ \\
\hline $\mathrm{C}(7)-\mathrm{N}(1)$ & $1.363(4)$ \\
\hline $\mathrm{C}(8)-\mathrm{C}(9)$ & $1.352(5)$ \\
\hline $\mathrm{C}(8)-\mathrm{H}(8)$ & 0.9300 \\
\hline$C(9)-C(10)$ & $1.392(5)$ \\
\hline $\mathrm{C}(9)-\mathrm{H}(9)$ & 0.9300 \\
\hline $\mathrm{C}(10)-\mathrm{N}(1)$ & $1.321(4)$ \\
\hline $\mathrm{C}(10)-\mathrm{H}(10)$ & 0.9300 \\
\hline $\mathrm{O}(1)-\mathrm{C}(11)$ & $1.217(4)$ \\
\hline $\mathrm{C}(11)-\mathrm{O}(2)$ & $1.335(4)$ \\
\hline $\mathrm{C}(11)-\mathrm{C}(13)$ & $1.443(5)$ \\
\hline $\mathrm{O}(2)-\mathrm{C}(12)$ & $1.440(4)$ \\
\hline $\mathrm{C}(12)-\mathrm{H}(12 \mathrm{~A})$ & 0.9600 \\
\hline $\mathrm{C}(12)-\mathrm{H}(12 \mathrm{~B})$ & 0.9600 \\
\hline $\mathrm{C}(12)-\mathrm{H}(12 \mathrm{C})$ & 0.9600 \\
\hline $\mathrm{C}(13)-\mathrm{C}(32)$ & $1.413(5)$ \\
\hline $\mathrm{C}(13)-\mathrm{P}(1)$ & $1.772(4)$ \\
\hline$P(1)-C(20)$ & $1.796(3)$ \\
\hline$P(1)-C(14)$ & $1.811(4)$ \\
\hline$P(1)-C(26)$ & $1.812(4)$ \\
\hline $\mathrm{C}(14)-\mathrm{C}(15)$ & $1.371(5)$ \\
\hline $\mathrm{C}(14)-\mathrm{C}(19)$ & $1.388(5)$ \\
\hline$C(15)-C(16)$ & $1.369(5)$ \\
\hline $\mathrm{C}(15)-\mathrm{H}(15)$ & 0.9300 \\
\hline $\mathrm{C}(16)-\mathrm{C}(17)$ & $1.387(5)$ \\
\hline $\mathrm{C}(16)-\mathrm{H}(16)$ & 0.9300 \\
\hline $\mathrm{C}(17)-\mathrm{C}(18)$ & $1.389(5)$ \\
\hline $\mathrm{C}(17)-\mathrm{H}(17)$ & 0.9300 \\
\hline $\mathrm{C}(18)-\mathrm{C}(19)$ & $1.349(5)$ \\
\hline $\mathrm{C}(18)-\mathrm{H}(18)$ & 0.9300 \\
\hline C(19)-H(19) & 0.9300 \\
\hline $\mathrm{C}(20)-\mathrm{C}(21)$ & $1.399(5)$ \\
\hline $\mathrm{C}(20)-\mathrm{C}(25)$ & $1.401(5)$ \\
\hline $\mathrm{C}(21)-\mathrm{C}(22)$ & $1.382(5)$ \\
\hline $\mathrm{C}(21)-\mathrm{H}(21)$ & 0.9300 \\
\hline $\mathrm{C}(22)-\mathrm{C}(23)$ & $1.378(5)$ \\
\hline $\mathrm{C}(22)-\mathrm{H}(22)$ & 0.9300 \\
\hline $\mathrm{C}(23)-\mathrm{C}(24)$ & $1.372(5)$ \\
\hline $\mathrm{C}(23)-\mathrm{H}(23)$ & 0.9300 \\
\hline
\end{tabular}




\begin{tabular}{|c|c|}
\hline $\mathrm{C}(24)-\mathrm{C}(25)$ & $1.391(5)$ \\
\hline $\mathrm{C}(24)-\mathrm{H}(24)$ & 0.9300 \\
\hline $\mathrm{C}(25)-\mathrm{H}(25)$ & 0.9300 \\
\hline $\mathrm{C}(26)-\mathrm{C}(27)$ & $1.396(5)$ \\
\hline $\mathrm{C}(26)-\mathrm{C}(31)$ & $1.403(5)$ \\
\hline $\mathrm{C}(27)-\mathrm{C}(28)$ & $1.390(5)$ \\
\hline $\mathrm{C}(27)-\mathrm{H}(27)$ & 0.9300 \\
\hline $\mathrm{C}(28)-\mathrm{C}(29)$ & $1.379(5)$ \\
\hline $\mathrm{C}(28)-\mathrm{H}(28)$ & 0.9300 \\
\hline $\mathrm{C}(29)-\mathrm{C}(30)$ & $1.379(5)$ \\
\hline $\mathrm{C}(29)-\mathrm{H}(29)$ & 0.9300 \\
\hline $\mathrm{C}(30)-\mathrm{C}(31)$ & $1.371(5)$ \\
\hline $\mathrm{C}(30)-\mathrm{H}(30)$ & 0.9300 \\
\hline $\mathrm{C}(31)-\mathrm{H}(31)$ & 0.9300 \\
\hline $\mathrm{C}(32)-\mathrm{N}(2)$ & $1.306(4)$ \\
\hline $\mathrm{C}(32)-\mathrm{C}(33)$ & $1.528(5)$ \\
\hline $\mathrm{C}(33)-\mathrm{O}(3)$ & $1.198(4)$ \\
\hline $\mathrm{C}(33)-\mathrm{O}(4)$ & $1.322(5)$ \\
\hline $\mathrm{O}(4)-\mathrm{C}(34)$ & $1.436(4)$ \\
\hline $\mathrm{C}(34)-\mathrm{H}(34 \mathrm{~A})$ & 0.9600 \\
\hline $\mathrm{C}(34)-\mathrm{H}(34 \mathrm{~B})$ & 0.9600 \\
\hline $\mathrm{C}(34)-\mathrm{H}(34 \mathrm{C})$ & 0.9600 \\
\hline $\mathrm{N}(2)-\mathrm{C}(35)$ & $1.445(4)$ \\
\hline$C(35)-C(40)$ & $1.378(5)$ \\
\hline $\mathrm{C}(35)-\mathrm{C}(36)$ & $1.378(5)$ \\
\hline $\mathrm{C}(36)-\mathrm{C}(37)$ & $1.389(5)$ \\
\hline $\mathrm{C}(36)-\mathrm{H}(36)$ & 0.9300 \\
\hline $\mathrm{C}(37)-\mathrm{C}(38)$ & $1.380(5)$ \\
\hline $\mathrm{C}(37)-\mathrm{H}(37)$ & 0.9300 \\
\hline $\mathrm{C}(38)-\mathrm{C}(39)$ & $1.373(5)$ \\
\hline $\mathrm{C}(38)-\mathrm{H}(38)$ & 0.9300 \\
\hline $\mathrm{C}(39)-\mathrm{C}(40)$ & $1.390(5)$ \\
\hline $\mathrm{C}(39)-\mathrm{H}(39)$ & 0.9300 \\
\hline $\mathrm{C}(40)-\mathrm{H}(40)$ & 0.9300 \\
\hline $\operatorname{Pd}(2)-N(3)$ & $2.002(3)$ \\
\hline $\operatorname{Pd}(2)-C(41)$ & $2.006(3)$ \\
\hline $\operatorname{Pd}(2)-N(4)$ & $2.033(3)$ \\
\hline $\mathrm{Pd}(2)-\mathrm{O}(5)$ & $2.103(2)$ \\
\hline $\mathrm{C}(41)-\mathrm{C}(42)$ & $1.489(5)$ \\
\hline $\mathrm{C}(41)-\mathrm{H}(41 \mathrm{~A})$ & 0.9700 \\
\hline $\mathrm{C}(41)-\mathrm{H}(41 \mathrm{~B})$ & 0.9700 \\
\hline$C(42)-C(43)$ & $1.383(5)$ \\
\hline $\mathrm{C}(42)-\mathrm{C}(47)$ & $1.417(4)$ \\
\hline $\mathrm{C}(43)-\mathrm{C}(44)$ & $1.413(5)$ \\
\hline $\mathrm{C}(43)-\mathrm{H}(43)$ & 0.9300 \\
\hline $\mathrm{C}(44)-\mathrm{C}(45)$ & $1.362(5)$ \\
\hline $\mathrm{C}(44)-\mathrm{H}(44)$ & 0.9300 \\
\hline $\mathrm{C}(45)-\mathrm{C}(46)$ & $1.414(5)$ \\
\hline $\mathrm{C}(45)-\mathrm{H}(45)$ & 0.9300 \\
\hline $\mathrm{C}(46)-\mathrm{C}(48)$ & $1.418(5)$ \\
\hline $\mathrm{C}(46)-\mathrm{C}(47)$ & $1.431(5)$ \\
\hline $\mathrm{C}(47)-\mathrm{N}(3)$ & $1.383(4)$ \\
\hline $\mathrm{C}(48)-\mathrm{C}(49)$ & $1.365(5)$ \\
\hline $\mathrm{C}(48)-\mathrm{H}(48)$ & 0.9300 \\
\hline $\mathrm{C}(49)-\mathrm{C}(50)$ & $1.410(5)$ \\
\hline $\mathrm{C}(49)-\mathrm{H}(49)$ & 0.9300 \\
\hline $\mathrm{C}(50)-\mathrm{N}(3)$ & $1.321(4)$ \\
\hline $\mathrm{C}(50)-\mathrm{H}(50)$ & 0.9300 \\
\hline
\end{tabular}




\begin{tabular}{|c|c|}
\hline $\mathrm{O}(5)-\mathrm{C}(51)$ & $1.237(4)$ \\
\hline $\mathrm{C}(51)-\mathrm{O}(6)$ & $1.341(4)$ \\
\hline $\mathrm{C}(51)-\mathrm{C}(53)$ & $1.448(5)$ \\
\hline $\mathrm{O}(6)-\mathrm{C}(52)$ & $1.455(4)$ \\
\hline $\mathrm{C}(52)-\mathrm{H}(52 \mathrm{~A})$ & 0.9600 \\
\hline $\mathrm{C}(52)-\mathrm{H}(52 \mathrm{~B})$ & 0.9600 \\
\hline $\mathrm{C}(52)-\mathrm{H}(52 \mathrm{C})$ & 0.9600 \\
\hline $\mathrm{C}(53)-\mathrm{C}(72)$ & $1.431(4)$ \\
\hline $\mathrm{C}(53)-\mathrm{P}(2)$ & $1.767(4)$ \\
\hline$P(2)-C(66)$ & $1.785(4)$ \\
\hline$P(2)-C(60)$ & $1.789(4)$ \\
\hline $\mathrm{P}(2)-\mathrm{C}(54)$ & $1.797(4)$ \\
\hline $\mathrm{C}(54)-\mathrm{C}(59)$ & $1.381(5)$ \\
\hline $\mathrm{C}(54)-\mathrm{C}(55)$ & $1.396(5)$ \\
\hline$C(55)-C(56)$ & $1.366(5)$ \\
\hline $\mathrm{C}(55)-\mathrm{H}(55)$ & 0.9300 \\
\hline$C(56)-C(57)$ & $1.376(5)$ \\
\hline $\mathrm{C}(56)-\mathrm{H}(56)$ & 0.9300 \\
\hline $\mathrm{C}(57)-\mathrm{C}(58)$ & $1.384(5)$ \\
\hline $\mathrm{C}(57)-\mathrm{H}(57)$ & 0.9300 \\
\hline $\mathrm{C}(58)-\mathrm{C}(59)$ & $1.393(5)$ \\
\hline $\mathrm{C}(58)-\mathrm{H}(58)$ & 0.9300 \\
\hline $\mathrm{C}(59)-\mathrm{H}(59)$ & 0.9300 \\
\hline $\mathrm{C}(60)-\mathrm{C}(65)$ & $1.379(5)$ \\
\hline $\mathrm{C}(60)-\mathrm{C}(61)$ & $1.383(5)$ \\
\hline $\mathrm{C}(61)-\mathrm{C}(62)$ & $1.385(5)$ \\
\hline $\mathrm{C}(61)-\mathrm{H}(61)$ & 0.9300 \\
\hline $\mathrm{C}(62)-\mathrm{C}(63)$ & $1.398(5)$ \\
\hline $\mathrm{C}(62)-\mathrm{H}(62)$ & 0.9300 \\
\hline $\mathrm{C}(63)-\mathrm{C}(64)$ & $1.353(5)$ \\
\hline $\mathrm{C}(63)-\mathrm{H}(63)$ & 0.9300 \\
\hline $\mathrm{C}(64)-\mathrm{C}(65)$ & $1.372(5)$ \\
\hline $\mathrm{C}(64)-\mathrm{H}(64)$ & 0.9300 \\
\hline $\mathrm{C}(65)-\mathrm{H}(65)$ & 0.9300 \\
\hline $\mathrm{C}(66)-\mathrm{C}(71)$ & $1.372(5)$ \\
\hline$C(66)-C(67)$ & $1.382(5)$ \\
\hline $\mathrm{C}(67)-\mathrm{C}(68)$ & $1.387(5)$ \\
\hline $\mathrm{C}(67)-\mathrm{H}(67)$ & 0.9300 \\
\hline $\mathrm{C}(68)-\mathrm{C}(69)$ & $1.364(5)$ \\
\hline $\mathrm{C}(68)-\mathrm{H}(68)$ & 0.9300 \\
\hline $\mathrm{C}(69)-\mathrm{C}(70)$ & $1.380(5)$ \\
\hline $\mathrm{C}(69)-\mathrm{H}(69)$ & 0.9300 \\
\hline $\mathrm{C}(70)-\mathrm{C}(71)$ & $1.395(5)$ \\
\hline $\mathrm{C}(70)-\mathrm{H}(70)$ & 0.9300 \\
\hline $\mathrm{C}(71)-\mathrm{H}(71)$ & 0.9300 \\
\hline $\mathrm{C}(72)-\mathrm{N}(4)$ & $1.327(4)$ \\
\hline $\mathrm{C}(72)-\mathrm{C}(73)$ & $1.527(5)$ \\
\hline $\mathrm{C}(73)-\mathrm{O}(7)$ & $1.181(4)$ \\
\hline $\mathrm{C}(73)-\mathrm{O}(8)$ & $1.326(5)$ \\
\hline $\mathrm{O}(8)-\mathrm{C}(74)$ & $1.438(4)$ \\
\hline $\mathrm{C}(74)-\mathrm{H}(74 \mathrm{~A})$ & 0.9600 \\
\hline $\mathrm{C}(74)-\mathrm{H}(74 \mathrm{~B})$ & 0.9600 \\
\hline $\mathrm{C}(74)-\mathrm{H}(74 \mathrm{C})$ & 0.9600 \\
\hline $\mathrm{N}(4)-\mathrm{C}(75)$ & $1.450(4)$ \\
\hline $\mathrm{C}(75)-\mathrm{C}(80)$ & $1.387(5)$ \\
\hline $\mathrm{C}(75)-\mathrm{C}(76)$ & $1.395(5)$ \\
\hline $\mathrm{C}(76)-\mathrm{C}(77)$ & $1.386(5)$ \\
\hline $\mathrm{C}(76)-\mathrm{H}(76)$ & 0.9300 \\
\hline
\end{tabular}




\begin{tabular}{|c|c|}
\hline $\mathrm{C}(77)-\mathrm{C}(78)$ & $1.370(5)$ \\
\hline $\mathrm{C}(77)-\mathrm{H}(77)$ & 0.9300 \\
\hline $\mathrm{C}(78)-\mathrm{C}(79)$ & $1.396(5)$ \\
\hline $\mathrm{C}(78)-\mathrm{H}(78)$ & 0.9300 \\
\hline $\mathrm{C}(79)-\mathrm{C}(80)$ & $1.396(5)$ \\
\hline $\mathrm{C}(79)-\mathrm{H}(79)$ & 0.9300 \\
\hline $\mathrm{C}(80)-\mathrm{H}(80)$ & 0.9300 \\
\hline $\mathrm{Cl}(2)-\mathrm{O}(13)$ & $1.409(3)$ \\
\hline $\mathrm{Cl}(2)-\mathrm{O}(15)$ & $1.423(3)$ \\
\hline $\mathrm{Cl}(2)-\mathrm{O}(14)$ & $1.428(3)$ \\
\hline $\mathrm{Cl}(2)-\mathrm{O}(16)$ & $1.436(3)$ \\
\hline $\mathrm{Cl}(1)-\mathrm{O}(11)$ & $1.428(3)$ \\
\hline $\mathrm{Cl}(1)-\mathrm{O}(10)$ & $1.434(3)$ \\
\hline $\mathrm{Cl}(1)-\mathrm{O}(12)$ & $1.437(3)$ \\
\hline $\mathrm{Cl}(1)-\mathrm{O}(9)$ & $1.440(3)$ \\
\hline $\mathrm{N}(1)-\mathrm{Pd}(1)-\mathrm{C}(1)$ & $82.64(13)$ \\
\hline $\mathrm{N}(1)-\mathrm{Pd}(1)-\mathrm{N}(2)$ & $175.77(12)$ \\
\hline $\mathrm{C}(1)-\mathrm{Pd}(1)-\mathrm{N}(2)$ & $99.49(13)$ \\
\hline $\mathrm{N}(1)-\mathrm{Pd}(1)-\mathrm{O}(1)$ & $89.32(10)$ \\
\hline $\mathrm{C}(1)-\mathrm{Pd}(1)-\mathrm{O}(1)$ & $171.60(12)$ \\
\hline $\mathrm{N}(2)-\mathrm{Pd}(1)-\mathrm{O}(1)$ & $88.38(10)$ \\
\hline $\mathrm{C}(2)-\mathrm{C}(1)-\mathrm{Pd}(1)$ & $108.2(2)$ \\
\hline $\mathrm{C}(2)-\mathrm{C}(1)-\mathrm{H}(1 \mathrm{~A})$ & 110.1 \\
\hline $\mathrm{Pd}(1)-\mathrm{C}(1)-\mathrm{H}(1 \mathrm{~A})$ & 110.1 \\
\hline $\mathrm{C}(2)-\mathrm{C}(1)-\mathrm{H}(1 \mathrm{~B})$ & 110.1 \\
\hline $\mathrm{Pd}(1)-\mathrm{C}(1)-\mathrm{H}(1 \mathrm{~B})$ & 110.1 \\
\hline $\mathrm{H}(1 \mathrm{~A})-\mathrm{C}(1)-\mathrm{H}(1 \mathrm{~B})$ & 108.4 \\
\hline $\mathrm{C}(3)-\mathrm{C}(2)-\mathrm{C}(7)$ & $117.6(3)$ \\
\hline$C(3)-C(2)-C(1)$ & $126.0(3)$ \\
\hline$C(7)-C(2)-C(1)$ & $116.5(3)$ \\
\hline $\mathrm{C}(2)-\mathrm{C}(3)-\mathrm{C}(4)$ & $121.0(3)$ \\
\hline $\mathrm{C}(2)-\mathrm{C}(3)-\mathrm{H}(3)$ & 119.5 \\
\hline $\mathrm{C}(4)-\mathrm{C}(3)-\mathrm{H}(3)$ & 119.5 \\
\hline$C(5)-C(4)-C(3)$ & $121.1(3)$ \\
\hline $\mathrm{C}(5)-\mathrm{C}(4)-\mathrm{H}(4)$ & 119.5 \\
\hline $\mathrm{C}(3)-\mathrm{C}(4)-\mathrm{H}(4)$ & 119.5 \\
\hline$C(4)-C(5)-C(6)$ & $119.9(3)$ \\
\hline $\mathrm{C}(4)-\mathrm{C}(5)-\mathrm{H}(5)$ & 120.0 \\
\hline $\mathrm{C}(6)-\mathrm{C}(5)-\mathrm{H}(5)$ & 120.0 \\
\hline$C(8)-C(6)-C(7)$ & $117.5(3)$ \\
\hline$C(8)-C(6)-C(5)$ & $125.5(3)$ \\
\hline$C(7)-C(6)-C(5)$ & $116.9(3)$ \\
\hline$N(1)-C(7)-C(6)$ & $121.3(3)$ \\
\hline $\mathrm{N}(1)-\mathrm{C}(7)-\mathrm{C}(2)$ & $115.2(3)$ \\
\hline$C(6)-C(7)-C(2)$ & $123.4(3)$ \\
\hline$C(9)-C(8)-C(6)$ & $120.0(3)$ \\
\hline $\mathrm{C}(9)-\mathrm{C}(8)-\mathrm{H}(8)$ & 120.0 \\
\hline $\mathrm{C}(6)-\mathrm{C}(8)-\mathrm{H}(8)$ & 120.0 \\
\hline $\mathrm{C}(8)-\mathrm{C}(9)-\mathrm{C}(10)$ & $120.1(3)$ \\
\hline $\mathrm{C}(8)-\mathrm{C}(9)-\mathrm{H}(9)$ & 119.9 \\
\hline $\mathrm{C}(10)-\mathrm{C}(9)-\mathrm{H}(9)$ & 119.9 \\
\hline $\mathrm{N}(1)-\mathrm{C}(10)-\mathrm{C}(9)$ & $121.6(3)$ \\
\hline $\mathrm{N}(1)-\mathrm{C}(10)-\mathrm{H}(10)$ & 119.2 \\
\hline $\mathrm{C}(9)-\mathrm{C}(10)-\mathrm{H}(10)$ & 119.2 \\
\hline $\mathrm{C}(10)-\mathrm{N}(1)-\mathrm{C}(7)$ & $119.4(3)$ \\
\hline $\mathrm{C}(10)-\mathrm{N}(1)-\mathrm{Pd}(1)$ & $125.2(2)$ \\
\hline $\mathrm{C}(7)-\mathrm{N}(1)-\mathrm{Pd}(1)$ & $114.2(2)$ \\
\hline
\end{tabular}




\begin{tabular}{|c|c|}
\hline $\mathrm{C}(11)-\mathrm{O}(1)-\mathrm{Pd}(1)$ & $127.5(2)$ \\
\hline $\mathrm{O}(1)-\mathrm{C}(11)-\mathrm{O}(2)$ & $118.0(3)$ \\
\hline $\mathrm{O}(1)-\mathrm{C}(11)-\mathrm{C}(13)$ & $128.1(3)$ \\
\hline $\mathrm{O}(2)-\mathrm{C}(11)-\mathrm{C}(13)$ & $113.9(3)$ \\
\hline $\mathrm{C}(11)-\mathrm{O}(2)-\mathrm{C}(12)$ & $116.6(3)$ \\
\hline $\mathrm{O}(2)-\mathrm{C}(12)-\mathrm{H}(12 \mathrm{~A})$ & 109.5 \\
\hline $\mathrm{O}(2)-\mathrm{C}(12)-\mathrm{H}(12 \mathrm{~B})$ & 109.5 \\
\hline $\mathrm{H}(12 \mathrm{~A})-\mathrm{C}(12)-\mathrm{H}(12 \mathrm{~B})$ & 109.5 \\
\hline $\mathrm{O}(2)-\mathrm{C}(12)-\mathrm{H}(12 \mathrm{C})$ & 109.5 \\
\hline $\mathrm{H}(12 \mathrm{~A})-\mathrm{C}(12)-\mathrm{H}(12 \mathrm{C})$ & 109.5 \\
\hline $\mathrm{H}(12 \mathrm{~B})-\mathrm{C}(12)-\mathrm{H}(12 \mathrm{C})$ & 109.5 \\
\hline $\mathrm{C}(32)-\mathrm{C}(13)-\mathrm{C}(11)$ & $120.5(3)$ \\
\hline $\mathrm{C}(32)-\mathrm{C}(13)-\mathrm{P}(1)$ & $126.2(3)$ \\
\hline $\mathrm{C}(11)-\mathrm{C}(13)-\mathrm{P}(1)$ & $112.4(3)$ \\
\hline $\mathrm{C}(13)-\mathrm{P}(1)-\mathrm{C}(20)$ & $116.27(16)$ \\
\hline $\mathrm{C}(13)-\mathrm{P}(1)-\mathrm{C}(14)$ & $107.54(17)$ \\
\hline $\mathrm{C}(20)-\mathrm{P}(1)-\mathrm{C}(14)$ & $108.16(17)$ \\
\hline $\mathrm{C}(13)-\mathrm{P}(1)-\mathrm{C}(26)$ & $112.44(17)$ \\
\hline $\mathrm{C}(20)-\mathrm{P}(1)-\mathrm{C}(26)$ & $103.74(17)$ \\
\hline $\mathrm{C}(14)-\mathrm{P}(1)-\mathrm{C}(26)$ & $108.40(17)$ \\
\hline$C(15)-C(14)-C(19)$ & $119.3(4)$ \\
\hline $\mathrm{C}(15)-\mathrm{C}(14)-\mathrm{P}(1)$ & $117.4(3)$ \\
\hline $\mathrm{C}(19)-\mathrm{C}(14)-\mathrm{P}(1)$ & $123.1(3)$ \\
\hline$C(16)-C(15)-C(14)$ & $120.1(4)$ \\
\hline $\mathrm{C}(16)-\mathrm{C}(15)-\mathrm{H}(15)$ & 119.9 \\
\hline $\mathrm{C}(14)-\mathrm{C}(15)-\mathrm{H}(15)$ & 119.9 \\
\hline$C(15)-C(16)-C(17)$ & $119.7(4)$ \\
\hline $\mathrm{C}(15)-\mathrm{C}(16)-\mathrm{H}(16)$ & 120.1 \\
\hline $\mathrm{C}(17)-\mathrm{C}(16)-\mathrm{H}(16)$ & 120.1 \\
\hline$C(16)-C(17)-C(18)$ & $120.4(4)$ \\
\hline $\mathrm{C}(16)-\mathrm{C}(17)-\mathrm{H}(17)$ & 119.8 \\
\hline $\mathrm{C}(18)-\mathrm{C}(17)-\mathrm{H}(17)$ & 119.8 \\
\hline $\mathrm{C}(19)-\mathrm{C}(18)-\mathrm{C}(17)$ & $118.7(4)$ \\
\hline $\mathrm{C}(19)-\mathrm{C}(18)-\mathrm{H}(18)$ & 120.7 \\
\hline $\mathrm{C}(17)-\mathrm{C}(18)-\mathrm{H}(18)$ & 120.7 \\
\hline $\mathrm{C}(18)-\mathrm{C}(19)-\mathrm{C}(14)$ & $121.7(4)$ \\
\hline $\mathrm{C}(18)-\mathrm{C}(19)-\mathrm{H}(19)$ & 119.1 \\
\hline $\mathrm{C}(14)-\mathrm{C}(19)-\mathrm{H}(19)$ & 119.1 \\
\hline $\mathrm{C}(21)-\mathrm{C}(20)-\mathrm{C}(25)$ & $119.6(3)$ \\
\hline $\mathrm{C}(21)-\mathrm{C}(20)-\mathrm{P}(1)$ & $119.8(3)$ \\
\hline $\mathrm{C}(25)-\mathrm{C}(20)-\mathrm{P}(1)$ & $120.5(3)$ \\
\hline$C(22)-C(21)-C(20)$ & $119.8(4)$ \\
\hline $\mathrm{C}(22)-\mathrm{C}(21)-\mathrm{H}(21)$ & 120.1 \\
\hline $\mathrm{C}(20)-\mathrm{C}(21)-\mathrm{H}(21)$ & 120.1 \\
\hline $\mathrm{C}(23)-\mathrm{C}(22)-\mathrm{C}(21)$ & $120.2(4)$ \\
\hline $\mathrm{C}(23)-\mathrm{C}(22)-\mathrm{H}(22)$ & 119.9 \\
\hline $\mathrm{C}(21)-\mathrm{C}(22)-\mathrm{H}(22)$ & 119.9 \\
\hline $\mathrm{C}(24)-\mathrm{C}(23)-\mathrm{C}(22)$ & $120.8(4)$ \\
\hline $\mathrm{C}(24)-\mathrm{C}(23)-\mathrm{H}(23)$ & 119.6 \\
\hline $\mathrm{C}(22)-\mathrm{C}(23)-\mathrm{H}(23)$ & 119.6 \\
\hline $\mathrm{C}(23)-\mathrm{C}(24)-\mathrm{C}(25)$ & $120.2(4)$ \\
\hline $\mathrm{C}(23)-\mathrm{C}(24)-\mathrm{H}(24)$ & 119.9 \\
\hline $\mathrm{C}(25)-\mathrm{C}(24)-\mathrm{H}(24)$ & 119.9 \\
\hline $\mathrm{C}(24)-\mathrm{C}(25)-\mathrm{C}(20)$ & $119.4(4)$ \\
\hline $\mathrm{C}(24)-\mathrm{C}(25)-\mathrm{H}(25)$ & 120.3 \\
\hline $\mathrm{C}(20)-\mathrm{C}(25)-\mathrm{H}(25)$ & 120.3 \\
\hline$C(27)-C(26)-C(31)$ & $119.0(3)$ \\
\hline $\mathrm{C}(27)-\mathrm{C}(26)-\mathrm{P}(1)$ & $122.2(3)$ \\
\hline
\end{tabular}




\begin{tabular}{|c|c|}
\hline $\mathrm{C}(31)-\mathrm{C}(26)-\mathrm{P}(1)$ & $118.7(3)$ \\
\hline $\mathrm{C}(28)-\mathrm{C}(27)-\mathrm{C}(26)$ & 119.4(4) \\
\hline $\mathrm{C}(28)-\mathrm{C}(27)-\mathrm{H}(27)$ & 120.3 \\
\hline $\mathrm{C}(26)-\mathrm{C}(27)-\mathrm{H}(27)$ & 120.3 \\
\hline$C(29)-C(28)-C(27)$ & $120.6(4)$ \\
\hline $\mathrm{C}(29)-\mathrm{C}(28)-\mathrm{H}(28)$ & 119.7 \\
\hline $\mathrm{C}(27)-\mathrm{C}(28)-\mathrm{H}(28)$ & 119.7 \\
\hline$C(28)-C(29)-C(30)$ & $120.1(3)$ \\
\hline $\mathrm{C}(28)-\mathrm{C}(29)-\mathrm{H}(29)$ & 119.9 \\
\hline $\mathrm{C}(30)-\mathrm{C}(29)-\mathrm{H}(29)$ & 119.9 \\
\hline $\mathrm{C}(31)-\mathrm{C}(30)-\mathrm{C}(29)$ & $120.1(4)$ \\
\hline $\mathrm{C}(31)-\mathrm{C}(30)-\mathrm{H}(30)$ & 120.0 \\
\hline $\mathrm{C}(29)-\mathrm{C}(30)-\mathrm{H}(30)$ & 120.0 \\
\hline$C(30)-C(31)-C(26)$ & $120.7(4)$ \\
\hline $\mathrm{C}(30)-\mathrm{C}(31)-\mathrm{H}(31)$ & 119.7 \\
\hline $\mathrm{C}(26)-\mathrm{C}(31)-\mathrm{H}(31)$ & 119.7 \\
\hline $\mathrm{N}(2)-\mathrm{C}(32)-\mathrm{C}(13)$ & $128.0(3)$ \\
\hline$N(2)-C(32)-C(33)$ & $112.0(3)$ \\
\hline$C(13)-C(32)-C(33)$ & $120.1(3)$ \\
\hline $\mathrm{O}(3)-\mathrm{C}(33)-\mathrm{O}(4)$ & $127.1(4)$ \\
\hline $\mathrm{O}(3)-\mathrm{C}(33)-\mathrm{C}(32)$ & $122.9(4)$ \\
\hline $\mathrm{O}(4)-\mathrm{C}(33)-\mathrm{C}(32)$ & $109.9(3)$ \\
\hline $\mathrm{C}(33)-\mathrm{O}(4)-\mathrm{C}(34)$ & $115.6(3)$ \\
\hline $\mathrm{O}(4)-\mathrm{C}(34)-\mathrm{H}(34 \mathrm{~A})$ & 109.5 \\
\hline $\mathrm{O}(4)-\mathrm{C}(34)-\mathrm{H}(34 \mathrm{~B})$ & 109.5 \\
\hline $\mathrm{H}(34 \mathrm{~A})-\mathrm{C}(34)-\mathrm{H}(34 \mathrm{~B})$ & 109.5 \\
\hline $\mathrm{O}(4)-\mathrm{C}(34)-\mathrm{H}(34 \mathrm{C})$ & 109.5 \\
\hline $\mathrm{H}(34 \mathrm{~A})-\mathrm{C}(34)-\mathrm{H}(34 \mathrm{C})$ & 109.5 \\
\hline $\mathrm{H}(34 \mathrm{~B})-\mathrm{C}(34)-\mathrm{H}(34 \mathrm{C})$ & 109.5 \\
\hline $\mathrm{C}(32)-\mathrm{N}(2)-\mathrm{C}(35)$ & 119.2(3) \\
\hline $\mathrm{C}(32)-\mathrm{N}(2)-\mathrm{Pd}(1)$ & $125.8(2)$ \\
\hline $\mathrm{C}(35)-\mathrm{N}(2)-\mathrm{Pd}(1)$ & $114.9(2)$ \\
\hline$C(40)-C(35)-C(36)$ & $119.8(3)$ \\
\hline $\mathrm{C}(40)-\mathrm{C}(35)-\mathrm{N}(2)$ & $118.9(3)$ \\
\hline $\mathrm{C}(36)-\mathrm{C}(35)-\mathrm{N}(2)$ & $121.0(3)$ \\
\hline$C(35)-C(36)-C(37)$ & $120.2(4)$ \\
\hline $\mathrm{C}(35)-\mathrm{C}(36)-\mathrm{H}(36)$ & 119.9 \\
\hline $\mathrm{C}(37)-\mathrm{C}(36)-\mathrm{H}(36)$ & 119.9 \\
\hline $\mathrm{C}(38)-\mathrm{C}(37)-\mathrm{C}(36)$ & $119.6(4)$ \\
\hline $\mathrm{C}(38)-\mathrm{C}(37)-\mathrm{H}(37)$ & 120.2 \\
\hline $\mathrm{C}(36)-\mathrm{C}(37)-\mathrm{H}(37)$ & 120.2 \\
\hline$C(39)-C(38)-C(37)$ & $120.4(4)$ \\
\hline $\mathrm{C}(39)-\mathrm{C}(38)-\mathrm{H}(38)$ & 119.8 \\
\hline $\mathrm{C}(37)-\mathrm{C}(38)-\mathrm{H}(38)$ & 119.8 \\
\hline $\mathrm{C}(38)-\mathrm{C}(39)-\mathrm{C}(40)$ & $119.8(4)$ \\
\hline $\mathrm{C}(38)-\mathrm{C}(39)-\mathrm{H}(39)$ & 120.1 \\
\hline $\mathrm{C}(40)-\mathrm{C}(39)-\mathrm{H}(39)$ & 120.1 \\
\hline $\mathrm{C}(35)-\mathrm{C}(40)-\mathrm{C}(39)$ & $120.1(4)$ \\
\hline $\mathrm{C}(35)-\mathrm{C}(40)-\mathrm{H}(40)$ & 120.0 \\
\hline $\mathrm{C}(39)-\mathrm{C}(40)-\mathrm{H}(40)$ & 120.0 \\
\hline$N(3)-P d(2)-C(41)$ & $83.34(12)$ \\
\hline $\mathrm{N}(3)-\mathrm{Pd}(2)-\mathrm{N}(4)$ & $177.59(12)$ \\
\hline $\mathrm{C}(41)-\mathrm{Pd}(2)-\mathrm{N}(4)$ & $96.83(12)$ \\
\hline $\mathrm{N}(3)-\mathrm{Pd}(2)-\mathrm{O}(5)$ & $92.71(10)$ \\
\hline $\mathrm{C}(41)-\mathrm{Pd}(2)-\mathrm{O}(5)$ & $174.79(12)$ \\
\hline $\mathrm{N}(4)-\mathrm{Pd}(2)-\mathrm{O}(5)$ & $87.26(10)$ \\
\hline $\mathrm{C}(42)-\mathrm{C}(41)-\mathrm{Pd}(2)$ & $110.2(2)$ \\
\hline $\mathrm{C}(42)-\mathrm{C}(41)-\mathrm{H}(41 \mathrm{~A})$ & 109.6 \\
\hline
\end{tabular}




\begin{tabular}{|c|c|}
\hline $\mathrm{Pd}(2)-\mathrm{C}(41)-\mathrm{H}(41 \mathrm{~A})$ & 109.6 \\
\hline $\mathrm{C}(42)-\mathrm{C}(41)-\mathrm{H}(41 \mathrm{~B})$ & 109.6 \\
\hline $\mathrm{Pd}(2)-\mathrm{C}(41)-\mathrm{H}(41 \mathrm{~B})$ & 109.6 \\
\hline $\mathrm{H}(41 \mathrm{~A})-\mathrm{C}(41)-\mathrm{H}(41 \mathrm{~B})$ & 108.1 \\
\hline $\mathrm{C}(43)-\mathrm{C}(42)-\mathrm{C}(47)$ & 117.1(3) \\
\hline $\mathrm{C}(43)-\mathrm{C}(42)-\mathrm{C}(41)$ & $127.4(3)$ \\
\hline $\mathrm{C}(47)-\mathrm{C}(42)-\mathrm{C}(41)$ & $115.5(3)$ \\
\hline $\mathrm{C}(42)-\mathrm{C}(43)-\mathrm{C}(44)$ & $120.9(3)$ \\
\hline $\mathrm{C}(42)-\mathrm{C}(43)-\mathrm{H}(43)$ & 119.6 \\
\hline $\mathrm{C}(44)-\mathrm{C}(43)-\mathrm{H}(43)$ & 119.6 \\
\hline $\mathrm{C}(45)-\mathrm{C}(44)-\mathrm{C}(43)$ & $122.1(3)$ \\
\hline $\mathrm{C}(45)-\mathrm{C}(44)-\mathrm{H}(44)$ & 118.9 \\
\hline $\mathrm{C}(43)-\mathrm{C}(44)-\mathrm{H}(44)$ & 118.9 \\
\hline$C(44)-C(45)-C(46)$ & 119.7(3) \\
\hline $\mathrm{C}(44)-\mathrm{C}(45)-\mathrm{H}(45)$ & 120.2 \\
\hline $\mathrm{C}(46)-\mathrm{C}(45)-\mathrm{H}(45)$ & 120.2 \\
\hline$C(45)-C(46)-C(48)$ & $125.9(3)$ \\
\hline$C(45)-C(46)-C(47)$ & $117.8(3)$ \\
\hline $\mathrm{C}(48)-\mathrm{C}(46)-\mathrm{C}(47)$ & $116.3(3)$ \\
\hline $\mathrm{N}(3)-\mathrm{C}(47)-\mathrm{C}(42)$ & $115.7(3)$ \\
\hline $\mathrm{N}(3)-\mathrm{C}(47)-\mathrm{C}(46)$ & $122.1(3)$ \\
\hline$C(42)-C(47)-C(46)$ & $122.2(3)$ \\
\hline $\mathrm{C}(49)-\mathrm{C}(48)-\mathrm{C}(46)$ & $120.1(3)$ \\
\hline $\mathrm{C}(49)-\mathrm{C}(48)-\mathrm{H}(48)$ & 120.0 \\
\hline $\mathrm{C}(46)-\mathrm{C}(48)-\mathrm{H}(48)$ & 120.0 \\
\hline $\mathrm{C}(48)-\mathrm{C}(49)-\mathrm{C}(50)$ & $120.5(3)$ \\
\hline $\mathrm{C}(48)-\mathrm{C}(49)-\mathrm{H}(49)$ & 119.8 \\
\hline $\mathrm{C}(50)-\mathrm{C}(49)-\mathrm{H}(49)$ & 119.8 \\
\hline $\mathrm{N}(3)-\mathrm{C}(50)-\mathrm{C}(49)$ & $121.7(3)$ \\
\hline $\mathrm{N}(3)-\mathrm{C}(50)-\mathrm{H}(50)$ & 119.2 \\
\hline $\mathrm{C}(49)-\mathrm{C}(50)-\mathrm{H}(50)$ & 119.2 \\
\hline $\mathrm{C}(50)-\mathrm{N}(3)-\mathrm{C}(47)$ & $119.3(3)$ \\
\hline $\mathrm{C}(50)-\mathrm{N}(3)-\mathrm{Pd}(2)$ & $126.2(3)$ \\
\hline $\mathrm{C}(47)-\mathrm{N}(3)-\mathrm{Pd}(2)$ & $114.5(2)$ \\
\hline $\mathrm{C}(51)-\mathrm{O}(5)-\mathrm{Pd}(2)$ & $124.6(2)$ \\
\hline $\mathrm{O}(5)-\mathrm{C}(51)-\mathrm{O}(6)$ & $120.4(3)$ \\
\hline $\mathrm{O}(5)-\mathrm{C}(51)-\mathrm{C}(53)$ & $127.3(3)$ \\
\hline $\mathrm{O}(6)-\mathrm{C}(51)-\mathrm{C}(53)$ & $112.3(3)$ \\
\hline $\mathrm{C}(51)-\mathrm{O}(6)-\mathrm{C}(52)$ & $115.6(3)$ \\
\hline $\mathrm{O}(6)-\mathrm{C}(52)-\mathrm{H}(52 \mathrm{~A})$ & 109.5 \\
\hline $\mathrm{O}(6)-\mathrm{C}(52)-\mathrm{H}(52 \mathrm{~B})$ & 109.5 \\
\hline $\mathrm{H}(52 \mathrm{~A})-\mathrm{C}(52)-\mathrm{H}(52 \mathrm{~B})$ & 109.5 \\
\hline $\mathrm{O}(6)-\mathrm{C}(52)-\mathrm{H}(52 \mathrm{C})$ & 109.5 \\
\hline $\mathrm{H}(52 \mathrm{~A})-\mathrm{C}(52)-\mathrm{H}(52 \mathrm{C})$ & 109.5 \\
\hline $\mathrm{H}(52 \mathrm{~B})-\mathrm{C}(52)-\mathrm{H}(52 \mathrm{C})$ & 109.5 \\
\hline$C(72)-C(53)-C(51)$ & $119.7(3)$ \\
\hline $\mathrm{C}(72)-\mathrm{C}(53)-\mathrm{P}(2)$ & $124.0(3)$ \\
\hline $\mathrm{C}(51)-\mathrm{C}(53)-\mathrm{P}(2)$ & $115.3(2)$ \\
\hline$C(53)-P(2)-C(66)$ & $111.85(16)$ \\
\hline$C(53)-P(2)-C(60)$ & $111.38(17)$ \\
\hline $\mathrm{C}(66)-\mathrm{P}(2)-\mathrm{C}(60)$ & $109.12(16)$ \\
\hline $\mathrm{C}(53)-\mathrm{P}(2)-\mathrm{C}(54)$ & $113.76(16)$ \\
\hline $\mathrm{C}(66)-\mathrm{P}(2)-\mathrm{C}(54)$ & $103.56(16)$ \\
\hline $\mathrm{C}(60)-\mathrm{P}(2)-\mathrm{C}(54)$ & $106.73(16)$ \\
\hline$C(59)-C(54)-C(55)$ & $118.7(3)$ \\
\hline $\mathrm{C}(59)-\mathrm{C}(54)-\mathrm{P}(2)$ & $123.7(3)$ \\
\hline $\mathrm{C}(55)-\mathrm{C}(54)-\mathrm{P}(2)$ & $117.5(3)$ \\
\hline $\mathrm{C}(56)-\mathrm{C}(55)-\mathrm{C}(54)$ & 121.2(3) \\
\hline
\end{tabular}




\begin{tabular}{|c|c|}
\hline $\mathrm{C}(56)-\mathrm{C}(55)-\mathrm{H}(55)$ & 119.4 \\
\hline $\mathrm{C}(54)-\mathrm{C}(55)-\mathrm{H}(55)$ & 119.4 \\
\hline$C(55)-C(56)-C(57)$ & $119.7(4)$ \\
\hline $\mathrm{C}(55)-\mathrm{C}(56)-\mathrm{H}(56)$ & 120.1 \\
\hline $\mathrm{C}(57)-\mathrm{C}(56)-\mathrm{H}(56)$ & 120.1 \\
\hline $\mathrm{C}(56)-\mathrm{C}(57)-\mathrm{C}(58)$ & $120.4(4)$ \\
\hline $\mathrm{C}(56)-\mathrm{C}(57)-\mathrm{H}(57)$ & 119.8 \\
\hline $\mathrm{C}(58)-\mathrm{C}(57)-\mathrm{H}(57)$ & 119.8 \\
\hline $\mathrm{C}(57)-\mathrm{C}(58)-\mathrm{C}(59)$ & $119.6(4)$ \\
\hline $\mathrm{C}(57)-\mathrm{C}(58)-\mathrm{H}(58)$ & 120.2 \\
\hline $\mathrm{C}(59)-\mathrm{C}(58)-\mathrm{H}(58)$ & 120.2 \\
\hline $\mathrm{C}(54)-\mathrm{C}(59)-\mathrm{C}(58)$ & $120.2(3)$ \\
\hline $\mathrm{C}(54)-\mathrm{C}(59)-\mathrm{H}(59)$ & 119.9 \\
\hline $\mathrm{C}(58)-\mathrm{C}(59)-\mathrm{H}(59)$ & 119.9 \\
\hline$C(65)-C(60)-C(61)$ & $119.0(4)$ \\
\hline $\mathrm{C}(65)-\mathrm{C}(60)-\mathrm{P}(2)$ & $122.1(3)$ \\
\hline $\mathrm{C}(61)-\mathrm{C}(60)-\mathrm{P}(2)$ & $118.9(3)$ \\
\hline$C(60)-C(61)-C(62)$ & $119.9(4)$ \\
\hline $\mathrm{C}(60)-\mathrm{C}(61)-\mathrm{H}(61)$ & 120.1 \\
\hline $\mathrm{C}(62)-\mathrm{C}(61)-\mathrm{H}(61)$ & 120.1 \\
\hline $\mathrm{C}(61)-\mathrm{C}(62)-\mathrm{C}(63)$ & $119.7(4)$ \\
\hline $\mathrm{C}(61)-\mathrm{C}(62)-\mathrm{H}(62)$ & 120.1 \\
\hline $\mathrm{C}(63)-\mathrm{C}(62)-\mathrm{H}(62)$ & 120.1 \\
\hline $\mathrm{C}(64)-\mathrm{C}(63)-\mathrm{C}(62)$ & $119.9(4)$ \\
\hline $\mathrm{C}(64)-\mathrm{C}(63)-\mathrm{H}(63)$ & 120.0 \\
\hline $\mathrm{C}(62)-\mathrm{C}(63)-\mathrm{H}(63)$ & 120.0 \\
\hline$C(63)-C(64)-C(65)$ & $120.3(4)$ \\
\hline $\mathrm{C}(63)-\mathrm{C}(64)-\mathrm{H}(64)$ & 119.9 \\
\hline $\mathrm{C}(65)-\mathrm{C}(64)-\mathrm{H}(64)$ & 119.9 \\
\hline$C(64)-C(65)-C(60)$ & $121.2(4)$ \\
\hline $\mathrm{C}(64)-\mathrm{C}(65)-\mathrm{H}(65)$ & 119.4 \\
\hline $\mathrm{C}(60)-\mathrm{C}(65)-\mathrm{H}(65)$ & 119.4 \\
\hline $\mathrm{C}(71)-\mathrm{C}(66)-\mathrm{C}(67)$ & 119.1(3) \\
\hline $\mathrm{C}(71)-\mathrm{C}(66)-\mathrm{P}(2)$ & $122.3(3)$ \\
\hline $\mathrm{C}(67)-\mathrm{C}(66)-\mathrm{P}(2)$ & $118.5(3)$ \\
\hline$C(66)-C(67)-C(68)$ & $121.3(4)$ \\
\hline $\mathrm{C}(66)-\mathrm{C}(67)-\mathrm{H}(67)$ & 119.4 \\
\hline $\mathrm{C}(68)-\mathrm{C}(67)-\mathrm{H}(67)$ & 119.4 \\
\hline $\mathrm{C}(69)-\mathrm{C}(68)-\mathrm{C}(67)$ & $119.3(4)$ \\
\hline $\mathrm{C}(69)-\mathrm{C}(68)-\mathrm{H}(68)$ & 120.3 \\
\hline $\mathrm{C}(67)-\mathrm{C}(68)-\mathrm{H}(68)$ & 120.3 \\
\hline $\mathrm{C}(68)-\mathrm{C}(69)-\mathrm{C}(70)$ & $120.2(4)$ \\
\hline $\mathrm{C}(68)-\mathrm{C}(69)-\mathrm{H}(69)$ & 119.9 \\
\hline $\mathrm{C}(70)-\mathrm{C}(69)-\mathrm{H}(69)$ & 119.9 \\
\hline $\mathrm{C}(69)-\mathrm{C}(70)-\mathrm{C}(71)$ & $120.4(4)$ \\
\hline $\mathrm{C}(69)-\mathrm{C}(70)-\mathrm{H}(70)$ & 119.8 \\
\hline $\mathrm{C}(71)-\mathrm{C}(70)-\mathrm{H}(70)$ & 119.8 \\
\hline $\mathrm{C}(66)-\mathrm{C}(71)-\mathrm{C}(70)$ & $119.7(4)$ \\
\hline $\mathrm{C}(66)-\mathrm{C}(71)-\mathrm{H}(71)$ & 120.1 \\
\hline $\mathrm{C}(70)-\mathrm{C}(71)-\mathrm{H}(71)$ & 120.1 \\
\hline $\mathrm{N}(4)-\mathrm{C}(72)-\mathrm{C}(53)$ & $125.9(3)$ \\
\hline $\mathrm{N}(4)-\mathrm{C}(72)-\mathrm{C}(73)$ & $114.4(3)$ \\
\hline $\mathrm{C}(53)-\mathrm{C}(72)-\mathrm{C}(73)$ & $119.6(3)$ \\
\hline $\mathrm{O}(7)-\mathrm{C}(73)-\mathrm{O}(8)$ & $127.5(4)$ \\
\hline $\mathrm{O}(7)-\mathrm{C}(73)-\mathrm{C}(72)$ & $123.5(4)$ \\
\hline $\mathrm{O}(8)-\mathrm{C}(73)-\mathrm{C}(72)$ & $109.0(3)$ \\
\hline $\mathrm{C}(73)-\mathrm{O}(8)-\mathrm{C}(74)$ & $113.7(3)$ \\
\hline $\mathrm{O}(8)-\mathrm{C}(74)-\mathrm{H}(74 \mathrm{~A})$ & 109.5 \\
\hline
\end{tabular}




$\begin{array}{ll}\mathrm{O}(8)-\mathrm{C}(74)-\mathrm{H}(74 \mathrm{~B}) & 109.5 \\ \mathrm{H}(74 \mathrm{~A})-\mathrm{C}(74)-\mathrm{H}(74 \mathrm{~B}) & 109.5 \\ \mathrm{O}(8)-\mathrm{C}(74)-\mathrm{H}(74 \mathrm{C}) & 109.5 \\ \mathrm{H}(74 \mathrm{~A})-\mathrm{C}(74)-\mathrm{H}(74 \mathrm{C}) & 109.5 \\ \mathrm{H}(74 \mathrm{~B})-\mathrm{C}(74)-\mathrm{H}(74 \mathrm{C}) & 109.5 \\ \mathrm{C}(72)-\mathrm{N}(4)-\mathrm{C}(75) & 115.9(3) \\ \mathrm{C}(72)-\mathrm{N}(4)-\mathrm{Pd}(2) & 124.7(2) \\ \mathrm{C}(75)-\mathrm{N}(4)-\mathrm{Pd}(2) & 118.7(2) \\ \mathrm{C}(80)-\mathrm{C}(75)-\mathrm{C}(76) & 119.6(3) \\ \mathrm{C}(80)-\mathrm{C}(75)-\mathrm{N}(4) & 120.9(3) \\ \mathrm{C}(76)-\mathrm{C}(75)-\mathrm{N}(4) & 119.5(3) \\ \mathrm{C}(77)-\mathrm{C}(76)-\mathrm{C}(75) & 120.6(4) \\ \mathrm{C}(77)-\mathrm{C}(76)-\mathrm{H}(76) & 119.7 \\ \mathrm{C}(75)-\mathrm{C}(76)-\mathrm{H}(76) & 119.7 \\ \mathrm{C}(78)-\mathrm{C}(77)-\mathrm{C}(76) & 120.3(4) \\ \mathrm{C}(78)-\mathrm{C}(77)-\mathrm{H}(77) & 119.9 \\ \mathrm{C}(76)-\mathrm{C}(77)-\mathrm{H}(77) & 119.9 \\ \mathrm{C}(77)-\mathrm{C}(78)-\mathrm{C}(79) & 119.3(4) \\ \mathrm{C}(77)-\mathrm{C}(78)-\mathrm{H}(78) & 120.3 \\ \mathrm{C}(79)-\mathrm{C}(78)-\mathrm{H}(78) & 120.3 \\ \mathrm{C}(78)-\mathrm{C}(79)-\mathrm{C}(80) & 121.1(4) \\ \mathrm{C}(78)-\mathrm{C}(79)-\mathrm{H}(79) & 119.5 \\ \mathrm{C}(80)-\mathrm{C}(79)-\mathrm{H}(79) & 119.5 \\ \mathrm{C}(75)-\mathrm{C}(80)-\mathrm{C}(79) & 119.0(4) \\ \mathrm{C}(75)-\mathrm{C}(80)-\mathrm{H}(80) & 120.5 \\ \mathrm{C}(79)-\mathrm{C}(80)-\mathrm{H}(80) & 120.5 \\ \mathrm{O}(13)-\mathrm{Cl}(2)-\mathrm{O}(15) & 109.85(19) \\ \mathrm{O}(13)-\mathrm{Cl}(2)-\mathrm{O}(14) & 109.5(2) \\ \mathrm{O}(15)-\mathrm{Cl}(2)-\mathrm{O}(14) & 109.2(2) \\ \mathrm{O}(13)-\mathrm{Cl}(2)-\mathrm{O}(16) & 109.73(19) \\ \mathrm{O}(15)-\mathrm{Cl}(2)-\mathrm{O}(16) & 110.31(17) \\ \mathrm{O}(14)-\mathrm{Cl}(2)-\mathrm{O}(16) & 108.24(18) \\ \mathrm{O}(11)-\mathrm{Cl}(1)-\mathrm{O}(10) & 109.44(17) \\ \mathrm{O}(11)-\mathrm{Cl}(1)-\mathrm{O}(12) & 109.30(18) \\ \mathrm{O}(10)-\mathrm{Cl}(1)-\mathrm{O}(12) & 108.97(17) \\ \mathrm{O}(11)-\mathrm{Cl}(1)-\mathrm{O}(9) & 110.71(19) \\ \mathrm{O}(10)-\mathrm{Cl}(1)-\mathrm{O}(9) & 108.79(19) \\ \mathrm{O}(12)-\mathrm{Cl}(1)-\mathrm{O}(9) & 109.60(16) \\ & \\ & \end{array}$

Symmetry transformations used to generate equivalent atoms: 
Table S9. Anisotropic displacement parameters $\left(\AA^{2} \times 10^{3}\right)$ for 3. The anisotropic displacement factor exponent takes the form: $-2 \pi^{2}\left[h^{2} a^{* 2} U^{11}+\ldots+2 h k a^{*} b^{*} U^{12}\right]$

\begin{tabular}{|c|c|c|c|c|c|c|}
\hline & $\mathrm{U}^{11}$ & $\mathrm{U}^{22}$ & $\mathrm{U}^{33}$ & $\mathrm{U}^{23}$ & $\mathrm{U}^{13}$ & $\mathrm{U}^{12}$ \\
\hline $\mathrm{Pd}(1)$ & $31(1)$ & $22(1)$ & $18(1)$ & 1(1) & $15(1)$ & 1(1) \\
\hline$C(1)$ & $32(2)$ & $27(2)$ & $17(2)$ & $1(2)$ & $12(2)$ & $4(2)$ \\
\hline$C(2)$ & 20(2) & $29(2)$ & $17(2)$ & $-6(2)$ & $9(2)$ & $-5(2)$ \\
\hline$C(3)$ & 29(2) & $35(2)$ & 21(2) & $-6(2)$ & $15(2)$ & $-1(2)$ \\
\hline $\mathrm{C}(4)$ & $30(2)$ & $33(2)$ & $30(2)$ & $-10(2)$ & $15(2)$ & $-3(2)$ \\
\hline $\mathrm{C}(5)$ & $30(2)$ & $29(2)$ & 30(2) & $-2(2)$ & $15(2)$ & $-7(2)$ \\
\hline $\mathrm{C}(6)$ & $25(2)$ & $27(2)$ & $24(2)$ & $-2(2)$ & $11(2)$ & $-7(2)$ \\
\hline $\mathrm{C}(7)$ & 20(2) & $21(2)$ & 20(2) & $-2(2)$ & $8(2)$ & $-3(2)$ \\
\hline $\mathrm{C}(8)$ & 41(3) & $33(2)$ & $24(2)$ & $-3(2)$ & $18(2)$ & $-15(2)$ \\
\hline $\mathrm{C}(9)$ & $54(3)$ & $33(3)$ & $28(2)$ & $-7(2)$ & $30(2)$ & $-13(2)$ \\
\hline $\mathrm{C}(10)$ & $42(3)$ & $27(2)$ & $27(2)$ & $-5(2)$ & $23(2)$ & $-4(2)$ \\
\hline $\mathrm{N}(1)$ & $33(2)$ & $23(2)$ & 19(2) & $-2(1)$ & $16(2)$ & $-2(2)$ \\
\hline $\mathrm{O}(1)$ & $47(2)$ & $24(2)$ & $25(2)$ & $-3(1)$ & $24(1)$ & $-8(1)$ \\
\hline $\mathrm{C}(11)$ & $26(2)$ & $23(2)$ & $23(2)$ & $0(2)$ & $13(2)$ & $4(2)$ \\
\hline $\mathrm{O}(2)$ & $33(2)$ & $29(2)$ & 16(1) & $0(1)$ & $13(1)$ & $-3(1)$ \\
\hline $\mathrm{C}(12)$ & $42(3)$ & $33(2)$ & $17(2)$ & $4(2)$ & $15(2)$ & $2(2)$ \\
\hline $\mathrm{C}(13)$ & 24(2) & 21(2) & $22(2)$ & $-1(2)$ & $12(2)$ & $-1(2)$ \\
\hline $\mathrm{P}(1)$ & $26(1)$ & $25(1)$ & $25(1)$ & $0(1)$ & $15(1)$ & $0(1)$ \\
\hline $\mathrm{C}(14)$ & 20(2) & $23(2)$ & $28(2)$ & $4(2)$ & $12(2)$ & $-3(2)$ \\
\hline$C(15)$ & $34(2)$ & $28(2)$ & $25(2)$ & $0(2)$ & $14(2)$ & $0(2)$ \\
\hline$C(16)$ & 34(3) & $32(2)$ & 39(3) & $7(2)$ & $17(2)$ & $2(2)$ \\
\hline $\mathrm{C}(17)$ & 23(2) & $42(3)$ & $27(2)$ & $4(2)$ & $0(2)$ & $-8(2)$ \\
\hline C(18) & $27(2)$ & $43(3)$ & $27(2)$ & $-6(2)$ & $8(2)$ & $1(2)$ \\
\hline $\mathrm{C}(19)$ & $24(2)$ & $32(2)$ & $33(2)$ & $-3(2)$ & $14(2)$ & 2(2) \\
\hline $\mathrm{C}(20)$ & $34(2)$ & $20(2)$ & $26(2)$ & $-1(2)$ & $19(2)$ & $0(2)$ \\
\hline $\mathrm{C}(21)$ & $36(3)$ & 41(3) & 41(3) & $13(2)$ & $25(2)$ & $11(2)$ \\
\hline $\mathrm{C}(22)$ & $57(3)$ & $33(3)$ & $53(3)$ & $18(2)$ & $37(3)$ & $19(2)$ \\
\hline $\mathrm{C}(23)$ & $57(3)$ & $22(2)$ & $44(3)$ & $3(2)$ & $37(3)$ & $-3(2)$ \\
\hline $\mathrm{C}(24)$ & $40(3)$ & $36(2)$ & $39(3)$ & $-7(2)$ & $27(2)$ & $-12(2)$ \\
\hline $\mathrm{C}(25)$ & $37(3)$ & $29(2)$ & $32(2)$ & $0(2)$ & $20(2)$ & $2(2)$ \\
\hline$C(26)$ & $26(2)$ & $35(2)$ & $17(2)$ & $7(2)$ & $12(2)$ & $6(2)$ \\
\hline $\mathrm{C}(27)$ & $25(2)$ & $28(2)$ & $18(2)$ & $3(2)$ & $8(2)$ & $1(2)$ \\
\hline $\mathrm{C}(28)$ & $22(2)$ & $46(3)$ & $22(2)$ & $10(2)$ & $12(2)$ & $5(2)$ \\
\hline $\mathrm{C}(29)$ & $26(2)$ & $36(3)$ & $22(2)$ & $1(2)$ & $10(2)$ & $9(2)$ \\
\hline $\mathrm{C}(30)$ & $33(2)$ & $27(2)$ & $31(2)$ & $-6(2)$ & $13(2)$ & $2(2)$ \\
\hline $\mathrm{C}(31)$ & 24(2) & $33(2)$ & $34(2)$ & $-2(2)$ & $12(2)$ & $-4(2)$ \\
\hline $\mathrm{C}(32)$ & 21(2) & $26(2)$ & 21(2) & $0(2)$ & $11(2)$ & $-1(2)$ \\
\hline C(33) & 40(3) & $26(2)$ & $25(2)$ & $-8(2)$ & $20(2)$ & $-8(2)$ \\
\hline $\mathrm{O}(3)$ & $48(2)$ & $37(2)$ & $45(2)$ & $-12(1)$ & $33(2)$ & $-14(2)$ \\
\hline $\mathrm{O}(4)$ & 43(2) & $42(2)$ & $33(2)$ & $20(2)$ & $8(2)$ & $-9(2)$ \\
\hline C(34) & $98(4)$ & 64(3) & $37(3)$ & $27(3)$ & $12(3)$ & $-30(3)$ \\
\hline $\mathrm{N}(2)$ & $25(2)$ & $25(2)$ & 18(2) & $3(1)$ & $13(2)$ & $2(1)$ \\
\hline C(35) & $31(2)$ & 21(2) & $17(2)$ & 2(2) & $14(2)$ & $-4(2)$ \\
\hline C(36) & $29(2)$ & $52(3)$ & 24(2) & $9(2)$ & $14(2)$ & $3(2)$ \\
\hline C(37) & $40(3)$ & $63(3)$ & $17(2)$ & $3(2)$ & $14(2)$ & $-7(2)$ \\
\hline C(38) & 61(3) & $40(3)$ & 33(3) & $-4(2)$ & $36(3)$ & $-6(2)$ \\
\hline C(39) & $39(3)$ & $38(3)$ & $31(2)$ & $6(2)$ & $19(2)$ & $11(2)$ \\
\hline $\mathrm{C}(40)$ & $30(2)$ & $36(2)$ & $20(2)$ & $7(2)$ & $11(2)$ & $6(2)$ \\
\hline $\operatorname{Pd}(2)$ & $28(1)$ & $20(1)$ & 20(1) & $0(1)$ & $14(1)$ & $2(1)$ \\
\hline $\mathrm{C}(41)$ & $31(2)$ & $25(2)$ & 19(2) & $0(2)$ & $14(2)$ & $5(2)$ \\
\hline $\mathrm{C}(42)$ & 18(2) & $30(2)$ & 19(2) & $-1(2)$ & $6(2)$ & $1(2)$ \\
\hline
\end{tabular}




\begin{tabular}{|c|c|c|c|c|c|c|}
\hline$C(43)$ & $32(2)$ & $32(2)$ & $14(2)$ & $-2(2)$ & $9(2)$ & $-3(2)$ \\
\hline$C(44)$ & $32(2)$ & $21(2)$ & $25(2)$ & $4(2)$ & $7(2)$ & $2(2)$ \\
\hline$C(45)$ & $28(2)$ & $21(2)$ & $27(2)$ & $2(2)$ & $8(2)$ & $7(2)$ \\
\hline$C(46)$ & $26(2)$ & $25(2)$ & $21(2)$ & $-8(2)$ & $8(2)$ & $-3(2)$ \\
\hline$C(47)$ & $26(2)$ & $25(2)$ & $14(2)$ & $-4(2)$ & $8(2)$ & $-5(2)$ \\
\hline $\mathrm{C}(48)$ & $36(3)$ & $29(2)$ & $37(3)$ & $-9(2)$ & $21(2)$ & $2(2)$ \\
\hline$C(49)$ & $52(3)$ & $31(2)$ & $37(3)$ & $-1(2)$ & $33(2)$ & $4(2)$ \\
\hline$C(50)$ & $44(3)$ & $31(2)$ & $28(2)$ & $5(2)$ & $24(2)$ & $5(2)$ \\
\hline $\mathrm{N}(3)$ & $32(2)$ & $24(2)$ & $19(2)$ & $0(1)$ & $14(2)$ & $1(2)$ \\
\hline $\mathrm{O}(5)$ & $44(2)$ & $23(2)$ & $24(2)$ & $4(1)$ & $20(1)$ & $6(1)$ \\
\hline$C(51)$ & $21(2)$ & $32(2)$ & $20(2)$ & $1(2)$ & $11(2)$ & $-1(2)$ \\
\hline $\mathrm{O}(6)$ & $36(2)$ & $25(1)$ & $21(1)$ & $3(1)$ & $18(1)$ & $4(1)$ \\
\hline $\mathrm{C}(52)$ & $54(3)$ & $33(2)$ & $25(2)$ & $1(2)$ & $29(2)$ & $4(2)$ \\
\hline$C(53)$ & $26(2)$ & $22(2)$ & $18(2)$ & $0(2)$ & $12(2)$ & $2(2)$ \\
\hline $\mathrm{P}(2)$ & $24(1)$ & $22(1)$ & $20(1)$ & $1(1)$ & $11(1)$ & $2(1)$ \\
\hline$C(54)$ & $27(2)$ & $25(2)$ & $14(2)$ & $4(2)$ & $11(2)$ & $3(2)$ \\
\hline$C(55)$ & $31(2)$ & $24(2)$ & $26(2)$ & $3(2)$ & $16(2)$ & $4(2)$ \\
\hline$C(56)$ & $38(2)$ & $23(2)$ & $30(2)$ & $0(2)$ & $18(2)$ & $-3(2)$ \\
\hline$C(57)$ & $30(2)$ & $39(3)$ & $28(2)$ & $1(2)$ & $13(2)$ & $-11(2)$ \\
\hline$C(58)$ & $28(2)$ & $36(2)$ & $30(2)$ & $3(2)$ & $15(2)$ & $3(2)$ \\
\hline C(59) & $28(2)$ & $23(2)$ & $23(2)$ & $2(2)$ & $10(2)$ & $-3(2)$ \\
\hline$C(60)$ & $23(2)$ & $25(2)$ & $22(2)$ & $-3(2)$ & $11(2)$ & $3(2)$ \\
\hline$C(61)$ & $36(3)$ & $42(3)$ & $28(2)$ & $8(2)$ & $12(2)$ & $7(2)$ \\
\hline$C(62)$ & $25(2)$ & $59(3)$ & $39(3)$ & $-4(2)$ & $6(2)$ & $-4(2)$ \\
\hline$C(63)$ & $41(3)$ & $52(3)$ & $21(2)$ & $8(2)$ & $9(2)$ & $22(2)$ \\
\hline C(64) & $40(3)$ & $35(2)$ & $30(3)$ & $3(2)$ & $16(2)$ & $5(2)$ \\
\hline$C(65)$ & $35(3)$ & $29(2)$ & $21(2)$ & $0(2)$ & $8(2)$ & $1(2)$ \\
\hline$C(66)$ & $22(2)$ & $22(2)$ & $22(2)$ & $4(2)$ & $10(2)$ & $4(2)$ \\
\hline$C(67)$ & $28(2)$ & $30(2)$ & $24(2)$ & $4(2)$ & $11(2)$ & $8(2)$ \\
\hline$C(68)$ & $38(3)$ & $35(2)$ & $24(2)$ & $1(2)$ & $14(2)$ & $3(2)$ \\
\hline C(69) & $45(3)$ & $36(3)$ & $34(3)$ & $-8(2)$ & $23(2)$ & $3(2)$ \\
\hline $\mathrm{C}(70)$ & $36(3)$ & $36(3)$ & $39(3)$ & 1(2) & $18(2)$ & $15(2)$ \\
\hline $\mathrm{C}(71)$ & $33(2)$ & $31(2)$ & $24(2)$ & $-1(2)$ & $13(2)$ & $3(2)$ \\
\hline$C(72)$ & $21(2)$ & $23(2)$ & $17(2)$ & $-3(2)$ & $7(2)$ & $-3(2)$ \\
\hline$C(73)$ & $30(3)$ & $18(2)$ & $43(3)$ & $2(2)$ & $26(2)$ & $-2(2)$ \\
\hline $\mathrm{O}(7)$ & $51(2)$ & $29(2)$ & $40(2)$ & $-5(1)$ & $36(2)$ & $-3(1)$ \\
\hline $\mathrm{O}(8)$ & $25(2)$ & $32(2)$ & $47(2)$ & $4(1)$ & $21(2)$ & $8(1)$ \\
\hline$C(74)$ & $37(3)$ & $42(3)$ & $83(4)$ & $6(3)$ & $38(3)$ & $10(2)$ \\
\hline $\mathrm{N}(4)$ & $26(2)$ & $26(2)$ & $18(2)$ & $3(1)$ & $14(2)$ & $1(1)$ \\
\hline$C(75)$ & $31(2)$ & $16(2)$ & $32(2)$ & $1(2)$ & $18(2)$ & $0(2)$ \\
\hline$C(76)$ & $31(3)$ & $29(2)$ & $29(2)$ & $5(2)$ & $12(2)$ & $-4(2)$ \\
\hline$C(77)$ & $28(2)$ & $33(2)$ & $49(3)$ & $3(2)$ & $17(2)$ & $-4(2)$ \\
\hline $\mathrm{C}(78)$ & $34(3)$ & $35(3)$ & $49(3)$ & $4(2)$ & $29(2)$ & 1(2) \\
\hline$C(79)$ & $43(3)$ & $26(2)$ & $31(2)$ & $2(2)$ & $25(2)$ & $2(2)$ \\
\hline $\mathrm{C}(80)$ & $28(2)$ & $22(2)$ & $25(2)$ & $1(2)$ & $12(2)$ & $2(2)$ \\
\hline $\mathrm{Cl}(2)$ & $38(1)$ & $35(1)$ & $38(1)$ & $6(1)$ & $21(1)$ & $10(1)$ \\
\hline $\mathrm{O}(13)$ & $43(2)$ & $57(2)$ & $67(2)$ & $-15(2)$ & $22(2)$ & $-4(2)$ \\
\hline $\mathrm{O}(14)$ & $64(2)$ & $95(3)$ & $37(2)$ & $13(2)$ & $24(2)$ & $35(2)$ \\
\hline $\mathrm{O}(15)$ & $66(2)$ & $31(2)$ & $112(3)$ & $0(2)$ & $62(2)$ & $10(2)$ \\
\hline $\mathrm{O}(16)$ & $58(2)$ & $45(2)$ & $53(2)$ & $12(2)$ & $42(2)$ & $16(2)$ \\
\hline $\mathrm{Cl}(1)$ & $50(1)$ & $28(1)$ & $27(1)$ & $0(1)$ & $24(1)$ & $-5(1)$ \\
\hline $\mathrm{O}(9)$ & $62(2)$ & $51(2)$ & $64(2)$ & $28(2)$ & $47(2)$ & $23(2)$ \\
\hline $\mathrm{O}(10)$ & $68(2)$ & $45(2)$ & $29(2)$ & $2(1)$ & $14(2)$ & $-28(2)$ \\
\hline $\mathrm{O}(11)$ & $86(3)$ & $58(2)$ & $37(2)$ & $-22(2)$ & $39(2)$ & $-37(2)$ \\
\hline $\mathrm{O}(12)$ & $55(2)$ & $43(2)$ & $33(2)$ & $0(1)$ & $27(2)$ & $10(2)$ \\
\hline
\end{tabular}


Table S10. Hydrogen coordinates ( $\left.\mathrm{x} 10^{4}\right)$ and isotropic displacement parameters $\left(\AA^{2} \times 10^{3}\right)$ for 3 .

\begin{tabular}{|c|c|c|c|c|}
\hline & $\mathrm{x}$ & $\mathrm{y}$ & $\mathrm{z}$ & $\mathrm{U}(\mathrm{eq})$ \\
\hline $\mathrm{H}(1 \mathrm{~A})$ & 9006 & 9950 & 7876 & 30 \\
\hline $\mathrm{H}(1 \mathrm{~B})$ & 9702 & 9716 & 8107 & 30 \\
\hline $\mathrm{H}(3)$ & 9424 & 11659 & 8213 & 32 \\
\hline $\mathrm{H}(4)$ & 9609 & 13041 & 7808 & 37 \\
\hline $\mathrm{H}(5)$ & 9669 & 13078 & 6840 & 35 \\
\hline $\mathrm{H}(8)$ & 9644 & 12148 & 5875 & 38 \\
\hline $\mathrm{H}(9)$ & 9607 & 10736 & 5426 & 41 \\
\hline $\mathrm{H}(10)$ & 9465 & 9425 & 5911 & 35 \\
\hline $\mathrm{H}(12 \mathrm{~A})$ & 8345 & 8477 & 5010 & 45 \\
\hline $\mathrm{H}(12 \mathrm{~B})$ & 8286 & 7655 & 4547 & 45 \\
\hline $\mathrm{H}(12 \mathrm{C})$ & 8930 & 7938 & 5080 & 45 \\
\hline $\mathrm{H}(15)$ & 7143 & 7037 & 5792 & 34 \\
\hline $\mathrm{H}(16)$ & 6316 & 7592 & 4908 & 41 \\
\hline $\mathrm{H}(17)$ & 6167 & 7149 & 3889 & 42 \\
\hline $\mathrm{H}(18)$ & 6805 & 6054 & 3763 & 40 \\
\hline H(19) & 7596 & 5472 & 4649 & 35 \\
\hline $\mathrm{H}(21)$ & 8746 & 4446 & 6914 & 44 \\
\hline $\mathrm{H}(22)$ & 8442 & 3308 & 7411 & 51 \\
\hline $\mathrm{H}(23)$ & 7453 & 3276 & 7298 & 43 \\
\hline $\mathrm{H}(24)$ & 6759 & 4372 & 6695 & 42 \\
\hline $\mathrm{H}(25)$ & 7030 & 5471 & 6137 & 37 \\
\hline $\mathrm{H}(27)$ & 9336 & 6130 & 6035 & 30 \\
\hline $\mathrm{H}(28)$ & 9911 & 5264 & 5655 & 35 \\
\hline $\mathrm{H}(29)$ & 9550 & 3872 & 5154 & 34 \\
\hline $\mathrm{H}(30)$ & 8639 & 3287 & 5079 & 37 \\
\hline $\mathrm{H}(31)$ & 8067 & 4118 & 5465 & 37 \\
\hline $\mathrm{H}(34 \mathrm{~A})$ & 8769 & 5967 & 8652 & 109 \\
\hline $\mathrm{H}(34 \mathrm{~B})$ & 9096 & 5132 & 8504 & 109 \\
\hline $\mathrm{H}(34 \mathrm{C})$ & 8385 & 5246 & 8126 & 109 \\
\hline $\mathrm{H}(36)$ & 9474 & 7941 & 8531 & 41 \\
\hline $\mathrm{H}(37)$ & 9337 & 8450 & 9409 & 47 \\
\hline $\mathrm{H}(38)$ & 8410 & 9078 & 9257 & 47 \\
\hline H(39) & 7611 & 9136 & 8246 & 41 \\
\hline $\mathrm{H}(40)$ & 7744 & 8601 & 7371 & 35 \\
\hline $\mathrm{H}(41 \mathrm{~A})$ & 6338 & 4959 & 2857 & 29 \\
\hline $\mathrm{H}(41 \mathrm{~B})$ & 5718 & 4471 & 2420 & 29 \\
\hline $\mathrm{H}(43)$ & 5710 & 6459 & 2155 & 32 \\
\hline $\mathrm{H}(44)$ & 5089 & 7670 & 2161 & 33 \\
\hline $\mathrm{H}(45)$ & 4599 & 7660 & 2805 & 33 \\
\hline $\mathrm{H}(48)$ & 4404 & 6787 & 3690 & 38 \\
\hline H(49) & 4669 & 5575 & 4394 & 42 \\
\hline $\mathrm{H}(50)$ & 5324 & 4432 & 4361 & 38 \\
\hline $\mathrm{H}(52 \mathrm{~A})$ & 6338 & 3165 & 5517 & 50 \\
\hline $\mathrm{H}(52 \mathrm{~B})$ & 6162 & 2300 & 5807 & 50 \\
\hline $\mathrm{H}(52 \mathrm{C})$ & 5660 & 2815 & 5217 & 50 \\
\hline $\mathrm{H}(55)$ & 5759 & -1087 & 4098 & 31 \\
\hline $\mathrm{H}(56)$ & 4882 & -1661 & 4098 & 35 \\
\hline $\mathrm{H}(57)$ & 4146 & -668 & 4107 & 38 \\
\hline $\mathrm{H}(58)$ & 4284 & 910 & 4101 & 37 \\
\hline $\mathrm{H}(59)$ & 5197 & 1490 & 4157 & 30 \\
\hline $\mathrm{H}(61)$ & 7482 & 1304 & 5081 & 43 \\
\hline
\end{tabular}




$\begin{array}{lrrrr}\mathrm{H}(62) & 8177 & 1000 & 6130 & 52 \\ \mathrm{H}(63) & 7977 & -172 & 6700 & 47 \\ \mathrm{H}(64) & 7080 & -970 & 6239 & 41 \\ \mathrm{H}(65) & 6385 & -653 & 5208 & 36 \\ \mathrm{H}(67) & 5686 & 278 & 2922 & 32 \\ \mathrm{H}(68) & 5808 & -670 & 2184 & 39 \\ \mathrm{H}(69) & 6647 & -1617 & 2511 & 43 \\ \mathrm{H}(70) & 7366 & -1615 & 3569 & 44 \\ \mathrm{H}(71) & 7240 & -669 & 4312 & 35 \\ \mathrm{H}(74 \mathrm{~A}) & 7784 & 363 & 3601 & 74 \\ \mathrm{H}(74 \mathrm{~B}) & 8287 & 658 & 4277 & 74 \\ \mathrm{H}(74 \mathrm{C}) & 8146 & 1285 & 3678 & 74 \\ \mathrm{H}(76) & 7718 & 3358 & 4038 & 36 \\ \mathrm{H}(77) & 8418 & 3710 & 3645 & 44 \\ \mathrm{H}(78) & 8101 & 3897 & 2559 & 42 \\ \mathrm{H}(79) & 7073 & 3729 & 1860 & 36 \\ \mathrm{H}(80) & 6365 & 3338 & 2243 & 30\end{array}$


Table S11. Crystal data and structure refinement for $100.5 \mathrm{CHCl}_{3}$.

Empirical formula

Formula weight

Temperature

Wavelength

Crystal system

Space group

Unit cell dimensions

Volume

$\mathrm{Z}$

Density (calculated)

Absorption coefficient

$\mathrm{F}(000)$

Crystal size

Theta range for data collection

Index ranges

Reflections collected

Independent reflections

Completeness to theta $=25.06^{\circ}$

Absorption correction

Max. and min. transmission

Refinement method

Data / restraints / parameters

Goodness-of-fit on $\mathrm{F}^{2}$

Final R indices [I $>2 \operatorname{sigma}(\mathrm{I})]$

$\mathrm{R}$ indices (all data)

Largest diff. peak and hole

\section{C60.50 H50 Cl3.50 N2 O8 P2 Pd2}

1331.84

100(2) K

$0.71073 \AA$

Monoclinic

$\mathrm{P} 2(1) / \mathrm{c}$

$\begin{array}{ll}\mathrm{a}=11.8913(11) \AA & \alpha=90^{\circ} . \\ \mathrm{b}=32.154(3) \AA & \beta=90.432(2)^{\circ} . \\ \mathrm{c}=15.7008(15) \AA & \gamma=90^{\circ} .\end{array}$

6003.1(10) $\AA^{3}$

4

$1.474 \mathrm{Mg} / \mathrm{m}^{3}$

$0.862 \mathrm{~mm}^{-1}$

2690

$0.37 \times 0.25 \times 0.25 \mathrm{~mm}^{3}$

2.30 to $25.06 \infty$.

$-10<=\mathrm{h}<=14,-29<=\mathrm{k}<=38,-18<=\mathrm{l}<=17$

24605

$8761[\mathrm{R}(\mathrm{int})=0.0791]$

$82.3 \%$

None

0.8133 and 0.7409

Full-matrix least-squares on $\mathrm{F}^{2}$

$8761 / 3 / 725$

0.991

$\mathrm{R} 1=0.0575, \mathrm{wR} 2=0.1367$

$\mathrm{R} 1=0.0850, \mathrm{wR} 2=0.1454$

1.696 and -0.628 e. $\AA^{-3}$ 
Table S12. Atomic coordinates ( $\mathrm{x} 10^{4}$ ) and equivalent isotropic displacement parameters $\left(\AA^{2} \times 10^{3}\right)$ for $100.5 \mathrm{CHCl}_{3}$. U(eq) is defined as one third of the trace of the orthogonalized $\mathrm{U}^{\mathrm{ij}}$ tensor.

\begin{tabular}{|c|c|c|c|c|}
\hline & $\mathrm{X}$ & $\mathrm{y}$ & $\mathrm{z}$ & $\mathrm{U}(\mathrm{eq})$ \\
\hline $\operatorname{Pd}(1)$ & $3888(1)$ & $3855(1)$ & $3494(1)$ & $24(1)$ \\
\hline $\operatorname{Pd}(2)$ & $2149(1)$ & $4495(1)$ & $2344(1)$ & $25(1)$ \\
\hline $\mathrm{Cl}(2)$ & $3456(1)$ & $3970(1)$ & $2062(1)$ & $29(1)$ \\
\hline $\mathrm{Cl}(1)$ & $2635(1)$ & $4428(1)$ & $3863(1)$ & $29(1)$ \\
\hline $\mathrm{P}(1)$ & $3178(2)$ & $2926(1)$ & 4001(1) & $29(1)$ \\
\hline $\mathrm{P}(2)$ & $2648(1)$ & $5349(1)$ & 1395(1) & $26(1)$ \\
\hline $\mathrm{O}(1)$ & $1356(4)$ & $2850(1)$ & 5191(3) & $35(1)$ \\
\hline $\mathrm{O}(2)$ & 1974(4) & $3306(1)$ & $6183(3)$ & $35(1)$ \\
\hline $\mathrm{O}(3)$ & 4463(4) & $3212(2)$ & $6550(3)$ & $39(1)$ \\
\hline $\mathrm{O}(4)$ & $3816(4)$ & $3868(1)$ & $6551(3)$ & $36(1)$ \\
\hline $\mathrm{O}(5)$ & $1019(4)$ & $6189(1)$ & 2691(3) & $38(1)$ \\
\hline $\mathrm{O}(6)$ & $2810(4)$ & $6144(1)$ & $2235(3)$ & $33(1)$ \\
\hline $\mathrm{O}(7)$ & $-980(4)$ & $5619(2)$ & $2446(3)$ & $39(1)$ \\
\hline $\mathrm{O}(8)$ & $-114(4)$ & $5535(1)$ & $3726(3)$ & $36(1)$ \\
\hline $\mathrm{N}(1)$ & $4368(4)$ & $3765(2)$ & $4727(3)$ & $26(1)$ \\
\hline $\mathrm{N}(2)$ & $878(4)$ & $4895(2)$ & $2566(3)$ & $26(1)$ \\
\hline $\mathrm{C}(1)$ & $4852(5)$ & $3378(2)$ & $3232(4)$ & $27(1)$ \\
\hline$C(2)$ & $4558(6)$ & $2989(2)$ & $3522(4)$ & $29(2)$ \\
\hline$C(3)$ & $5236(6)$ & $2642(2)$ & $3382(5)$ & $41(2)$ \\
\hline $\mathrm{C}(4)$ & $6238(6)$ & $2695(2)$ & $2967(5)$ & $49(2)$ \\
\hline $\mathrm{C}(5)$ & $6535(7)$ & $3092(2)$ & $2667(5)$ & $48(2)$ \\
\hline$C(6)$ & $5842(6)$ & $3422(2)$ & $2790(4)$ & $34(2)$ \\
\hline$C(7)$ & $2160(5)$ & $3037(2)$ & $3173(4)$ & $29(2)$ \\
\hline $\mathrm{C}(8)$ & $2236(6)$ & $2850(2)$ & $2386(5)$ & $37(2)$ \\
\hline $\mathrm{C}(9)$ & $1477(7)$ & 2943(2) & $1759(5)$ & $42(2)$ \\
\hline$C(10)$ & $625(7)$ & $3225(2)$ & $1906(5)$ & $45(2)$ \\
\hline $\mathrm{C}(11)$ & $552(7)$ & $3423(2)$ & $2694(5)$ & $46(2)$ \\
\hline$C(12)$ & $1325(6)$ & $3324(2)$ & $3327(5)$ & $35(2)$ \\
\hline$C(13)$ & $3091(6)$ & $2390(2)$ & $4343(4)$ & $30(2)$ \\
\hline$C(14)$ & $3680(6)$ & $2274(2)$ & $5046(5)$ & $36(2)$ \\
\hline$C(15)$ & $3650(6)$ & $1871(2)$ & $5348(5)$ & $43(2)$ \\
\hline$C(16)$ & $3007(7)$ & $1575(2)$ & 4906(5) & $50(2)$ \\
\hline $\mathrm{C}(17)$ & $2384(7)$ & 1691(2) & $4175(5)$ & $44(2)$ \\
\hline $\mathrm{C}(18)$ & $2420(6)$ & 2099(2) & $3896(5)$ & $39(2)$ \\
\hline$C(19)$ & $3008(6)$ & $3219(2)$ & 4936(4) & $30(2)$ \\
\hline$C(20)$ & $2051(6)$ & $3097(2)$ & $5421(4)$ & $32(2)$ \\
\hline $\mathrm{C}(21)$ & $963(6)$ & $3235(2)$ & $6642(5)$ & $43(2)$ \\
\hline $\mathrm{C}(22)$ & $3829(5)$ & $3512(2)$ & $5231(4)$ & $29(2)$ \\
\hline $\mathrm{C}(23)$ & $4076(6)$ & $3505(2)$ & $6181(4)$ & $31(2)$ \\
\hline $\mathrm{C}(24)$ & $3916(8)$ & $3870(2)$ & $7462(4)$ & $49(2)$ \\
\hline$C(25)$ & $5359(6)$ & $3983(2)$ & $4998(4)$ & $31(2)$ \\
\hline$C(26)$ & $6220(6)$ & $3774(2)$ & $5400(4)$ & $32(2)$ \\
\hline $\mathrm{C}(27)$ & $7164(6)$ & 3989(2) & $5670(4)$ & $37(2)$ \\
\hline $\mathrm{C}(28)$ & $7232(7)$ & $4414(2)$ & $5531(4)$ & $39(2)$ \\
\hline C(29) & $6359(6)$ & $4616(2)$ & $5096(4)$ & $39(2)$ \\
\hline$C(30)$ & $5442(6)$ & $4403(2)$ & 4834(4) & $30(2)$ \\
\hline $\mathrm{C}(31)$ & $1796(5)$ & $4565(2)$ & $1146(4)$ & $28(2)$ \\
\hline$C(32)$ & $2016(5)$ & $4948(2)$ & $750(4)$ & $28(2)$ \\
\hline $\mathrm{C}(33)$ & $1786(6)$ & $5006(2)$ & $-118(4)$ & $37(2)$ \\
\hline $\mathrm{C}(34)$ & $1311(6)$ & $4692(2)$ & $-584(5)$ & $39(2)$ \\
\hline $\mathrm{C}(35)$ & $1073(6)$ & $4318(2)$ & $-204(5)$ & $36(2)$ \\
\hline
\end{tabular}




\begin{tabular}{|c|c|c|c|c|}
\hline$C(36)$ & $1300(6)$ & $4247(2)$ & $642(4)$ & $33(2)$ \\
\hline C(37) & $2980(6)$ & $5759(2)$ & $660(4)$ & $31(2)$ \\
\hline $\mathrm{C}(38)$ & $2123(7)$ & $6019(2)$ & $367(5)$ & $44(2)$ \\
\hline C(39) & $2346(8)$ & $6324(3)$ & $-226(5)$ & $59(2)$ \\
\hline$C(40)$ & $3403(8)$ & $6366(2)$ & $-550(5)$ & $50(2)$ \\
\hline $\mathrm{C}(41)$ & $4234(7)$ & $6112(2)$ & $-270(5)$ & $49(2)$ \\
\hline$C(42)$ & $4032(6)$ & $5810(2)$ & $337(4)$ & $36(2)$ \\
\hline$C(43)$ & $3950(5)$ & $5163(2)$ & $1833(4)$ & $24(1)$ \\
\hline$C(44)$ & $4666(6)$ & $4914(2)$ & $1357(4)$ & $33(2)$ \\
\hline$C(45)$ & $5694(6)$ & $4790(2)$ & $1700(5)$ & $40(2)$ \\
\hline$C(46)$ & $5985(6)$ & $4903(2)$ & $2518(5)$ & $42(2)$ \\
\hline$C(47)$ & $5256(6)$ & $5147(2)$ & $3000(5)$ & $38(2)$ \\
\hline$C(48)$ & $4257(6)$ & $5279(2)$ & $2653(4)$ & $32(2)$ \\
\hline$C(49)$ & $1710(5)$ & $5549(2)$ & $2147(4)$ & $27(1)$ \\
\hline$C(50)$ & $1764(6)$ & $5980(2)$ & $2390(4)$ & $31(2)$ \\
\hline$C(51)$ & $2918(6)$ & $6585(2)$ & $2319(5)$ & $42(2)$ \\
\hline$C(52)$ & $859(5)$ & $5299(2)$ & $2523(4)$ & $25(1)$ \\
\hline$C(53)$ & $-195(6)$ & $5514(2)$ & $2871(4)$ & $32(2)$ \\
\hline$C(54)$ & $-1121(6)$ & $5687(2)$ & $4137(5)$ & $43(2)$ \\
\hline$C(55)$ & $-73(5)$ & $4654(2)$ & $2849(4)$ & $29(2)$ \\
\hline C(56) & $-813(6)$ & $4491(2)$ & $2243(5)$ & $35(2)$ \\
\hline$C(57)$ & $-1674(6)$ & $4227(2)$ & $2515(5)$ & $38(2)$ \\
\hline $\mathrm{C}(58)$ & $-1784(6)$ & $4124(2)$ & $3360(5)$ & $45(2)$ \\
\hline C(59) & $-1032(6)$ & $4302(2)$ & $3953(5)$ & $45(2)$ \\
\hline$C(60)$ & $-182(6)$ & $4558(2)$ & $3701(5)$ & $38(2)$ \\
\hline C(100) & $-1042(13)$ & $2649(3)$ & $4990(8)$ & $63(5)$ \\
\hline $\mathrm{Cl}(3)$ & $-968(5)$ & $2290(2)$ & $4166(4)$ & $96(2)$ \\
\hline $\mathrm{Cl}(4)$ & $-1499(4)$ & $3131(1)$ & $4530(3)$ & $58(1)$ \\
\hline $\mathrm{Cl}(5)$ & $-2002(5)$ & 2494(2) & $5766(4)$ & $94(2)$ \\
\hline
\end{tabular}


Table S13. Bond lengths $[\AA]$ and angles $\left[^{\circ}\right]$ for $100.5 \mathrm{CHCl}_{3}$.

\begin{tabular}{|c|c|}
\hline $\operatorname{Pd}(1)-C(1)$ & $1.960(6)$ \\
\hline $\operatorname{Pd}(1)-N(1)$ & $2.035(5)$ \\
\hline $\mathrm{Pd}(1)-\mathrm{Cl}(2)$ & $2.3314(17)$ \\
\hline $\mathrm{Pd}(1)-\mathrm{Cl}(1)$ & $2.4437(15)$ \\
\hline $\operatorname{Pd}(2)-C(31)$ & $1.937(7)$ \\
\hline $\operatorname{Pd}(2)-\mathrm{N}(2)$ & $2.017(5)$ \\
\hline $\mathrm{Pd}(2)-\mathrm{Cl}(2)$ & $2.3377(15)$ \\
\hline $\mathrm{Pd}(2)-\mathrm{Cl}(1)$ & $2.4590(17)$ \\
\hline $\mathrm{P}(1)-\mathrm{C}(19)$ & $1.757(7)$ \\
\hline $\mathrm{P}(1)-\mathrm{C}(7)$ & $1.806(7)$ \\
\hline$P(1)-C(13)$ & $1.807(6)$ \\
\hline $\mathrm{P}(1)-\mathrm{C}(2)$ & $1.822(6)$ \\
\hline $\mathrm{P}(2)-\mathrm{C}(49)$ & $1.753(6)$ \\
\hline$P(2)-C(43)$ & $1.791(7)$ \\
\hline$P(2)-C(32)$ & $1.798(7)$ \\
\hline $\mathrm{P}(2)-\mathrm{C}(37)$ & $1.798(6)$ \\
\hline $\mathrm{O}(1)-\mathrm{C}(20)$ & $1.201(8)$ \\
\hline $\mathrm{O}(2)-\mathrm{C}(20)$ & $1.374(8)$ \\
\hline $\mathrm{O}(2)-\mathrm{C}(21)$ & $1.424(7)$ \\
\hline $\mathrm{O}(3)-\mathrm{C}(23)$ & $1.196(8)$ \\
\hline $\mathrm{O}(4)-\mathrm{C}(23)$ & $1.341(8)$ \\
\hline $\mathrm{O}(4)-\mathrm{C}(24)$ & $1.435(9)$ \\
\hline $\mathrm{O}(5)-\mathrm{C}(50)$ & $1.211(8)$ \\
\hline $\mathrm{O}(6)-\mathrm{C}(50)$ & $1.375(8)$ \\
\hline $\mathrm{O}(6)-\mathrm{C}(51)$ & $1.431(8)$ \\
\hline $\mathrm{O}(7)-\mathrm{C}(53)$ & 1.192(9) \\
\hline $\mathrm{O}(8)-\mathrm{C}(53)$ & $1.347(8)$ \\
\hline $\mathrm{O}(8)-\mathrm{C}(54)$ & $1.449(8)$ \\
\hline $\mathrm{N}(1)-\mathrm{C}(22)$ & $1.307(8)$ \\
\hline $\mathrm{N}(1)-\mathrm{C}(25)$ & $1.433(9)$ \\
\hline $\mathrm{N}(2)-\mathrm{C}(52)$ & $1.301(8)$ \\
\hline$N(2)-C(55)$ & $1.443(8)$ \\
\hline$C(1)-C(2)$ & $1.377(9)$ \\
\hline$C(1)-C(6)$ & $1.379(9)$ \\
\hline$C(2)-C(3)$ & $1.395(9)$ \\
\hline $\mathrm{C}(3)-\mathrm{C}(4)$ & $1.373(10)$ \\
\hline$C(4)-C(5)$ & $1.406(10)$ \\
\hline$C(5)-C(6)$ & $1.359(10)$ \\
\hline $\mathrm{C}(7)-\mathrm{C}(8)$ & $1.378(9)$ \\
\hline$C(7)-C(12)$ & $1.380(9)$ \\
\hline $\mathrm{C}(8)-\mathrm{C}(9)$ & $1.364(11)$ \\
\hline $\mathrm{C}(9)-\mathrm{C}(10)$ & $1.380(11)$ \\
\hline $\mathrm{C}(10)-\mathrm{C}(11)$ & $1.395(11)$ \\
\hline $\mathrm{C}(11)-\mathrm{C}(12)$ & $1.385(11)$ \\
\hline $\mathrm{C}(13)-\mathrm{C}(14)$ & $1.355(10)$ \\
\hline $\mathrm{C}(13)-\mathrm{C}(18)$ & $1.413(10)$ \\
\hline$C(14)-C(15)$ & $1.380(10)$ \\
\hline$C(15)-C(16)$ & $1.401(12)$ \\
\hline $\mathrm{C}(16)-\mathrm{C}(17)$ & $1.410(12)$ \\
\hline $\mathrm{C}(17)-\mathrm{C}(18)$ & $1.386(10)$ \\
\hline $\mathrm{C}(19)-\mathrm{C}(20)$ & $1.430(9)$ \\
\hline $\mathrm{C}(19)-\mathrm{C}(22)$ & $1.431(10)$ \\
\hline $\mathrm{C}(22)-\mathrm{C}(23)$ & $1.518(10)$ \\
\hline $\mathrm{C}(25)-\mathrm{C}(26)$ & $1.373(10)$ \\
\hline $\mathrm{C}(25)-\mathrm{C}(30)$ & $1.378(9)$ \\
\hline
\end{tabular}




\begin{tabular}{|c|c|}
\hline $\mathrm{C}(26)-\mathrm{C}(27)$ & $1.381(10)$ \\
\hline $\mathrm{C}(27)-\mathrm{C}(28)$ & $1.387(10)$ \\
\hline $\mathrm{C}(28)-\mathrm{C}(29)$ & $1.398(11)$ \\
\hline $\mathrm{C}(29)-\mathrm{C}(30)$ & $1.351(10)$ \\
\hline $\mathrm{C}(31)-\mathrm{C}(32)$ & $1.406(9)$ \\
\hline $\mathrm{C}(31)-\mathrm{C}(36)$ & $1.418(9)$ \\
\hline $\mathrm{C}(32)-\mathrm{C}(33)$ & $1.400(10)$ \\
\hline $\mathrm{C}(33)-\mathrm{C}(34)$ & $1.365(10)$ \\
\hline$C(34)-C(35)$ & $1.372(10)$ \\
\hline $\mathrm{C}(35)-\mathrm{C}(36)$ & $1.373(10)$ \\
\hline $\mathrm{C}(37)-\mathrm{C}(42)$ & $1.364(9)$ \\
\hline $\mathrm{C}(37)-\mathrm{C}(38)$ & $1.393(10)$ \\
\hline $\mathrm{C}(38)-\mathrm{C}(39)$ & $1.379(11)$ \\
\hline$C(39)-C(40)$ & $1.366(12)$ \\
\hline$C(40)-C(41)$ & $1.353(12)$ \\
\hline $\mathrm{C}(41)-\mathrm{C}(42)$ & $1.381(10)$ \\
\hline $\mathrm{C}(43)-\mathrm{C}(48)$ & $1.387(9)$ \\
\hline $\mathrm{C}(43)-\mathrm{C}(44)$ & $1.392(9)$ \\
\hline C(44)-C(45) & $1.389(10)$ \\
\hline$C(45)-C(46)$ & $1.377(11)$ \\
\hline $\mathrm{C}(46)-\mathrm{C}(47)$ & $1.396(10)$ \\
\hline $\mathrm{C}(47)-\mathrm{C}(48)$ & $1.371(10)$ \\
\hline $\mathrm{C}(49)-\mathrm{C}(52)$ & $1.423(9)$ \\
\hline C(49)-C(50) & $1.441(9)$ \\
\hline $\mathrm{C}(52)-\mathrm{C}(53)$ & $1.536(9)$ \\
\hline $\mathrm{C}(55)-\mathrm{C}(60)$ & $1.380(10)$ \\
\hline$C(55)-C(56)$ & $1.394(10)$ \\
\hline$C(56)-C(57)$ & $1.398(10)$ \\
\hline$C(57)-C(58)$ & $1.375(11)$ \\
\hline $\mathrm{C}(58)-\mathrm{C}(59)$ & $1.408(12)$ \\
\hline $\mathrm{C}(59)-\mathrm{C}(60)$ & $1.364(10)$ \\
\hline $\mathrm{C}(100)-\mathrm{Cl}(3)$ & $1.739(12)$ \\
\hline $\mathrm{C}(100)-\mathrm{Cl}(5)$ & $1.749(11)$ \\
\hline $\mathrm{C}(100)-\mathrm{Cl}(4)$ & $1.792(12)$ \\
\hline $\mathrm{C}(1)-\mathrm{Pd}(1)-\mathrm{N}(1)$ & $85.9(2)$ \\
\hline $\mathrm{C}(1)-\mathrm{Pd}(1)-\mathrm{Cl}(2)$ & $92.71(19)$ \\
\hline $\mathrm{N}(1)-\mathrm{Pd}(1)-\mathrm{Cl}(2)$ & $176.32(15)$ \\
\hline $\mathrm{C}(1)-\mathrm{Pd}(1)-\mathrm{Cl}(1)$ & $177.36(18)$ \\
\hline $\mathrm{N}(1)-\mathrm{Pd}(1)-\mathrm{Cl}(1)$ & $92.81(15)$ \\
\hline $\mathrm{Cl}(2)-\mathrm{Pd}(1)-\mathrm{Cl}(1)$ & $88.73(6)$ \\
\hline $\mathrm{C}(31)-\mathrm{Pd}(2)-\mathrm{N}(2)$ & $86.3(2)$ \\
\hline $\mathrm{C}(31)-\mathrm{Pd}(2)-\mathrm{Cl}(2)$ & $92.33(19)$ \\
\hline $\mathrm{N}(2)-\mathrm{Pd}(2)-\mathrm{Cl}(2)$ & $173.08(16)$ \\
\hline $\mathrm{C}(31)-\mathrm{Pd}(2)-\mathrm{Cl}(1)$ & $178.0(2)$ \\
\hline $\mathrm{N}(2)-\mathrm{Pd}(2)-\mathrm{Cl}(1)$ & $93.36(15)$ \\
\hline $\mathrm{Cl}(2)-\mathrm{Pd}(2)-\mathrm{Cl}(1)$ & $88.23(5)$ \\
\hline $\mathrm{Pd}(1)-\mathrm{Cl}(2)-\mathrm{Pd}(2)$ & $94.29(5)$ \\
\hline $\mathrm{Pd}(1)-\mathrm{Cl}(1)-\mathrm{Pd}(2)$ & $88.56(5)$ \\
\hline $\mathrm{C}(19)-\mathrm{P}(1)-\mathrm{C}(7)$ & $114.5(3)$ \\
\hline $\mathrm{C}(19)-\mathrm{P}(1)-\mathrm{C}(13)$ & $104.9(3)$ \\
\hline $\mathrm{C}(7)-\mathrm{P}(1)-\mathrm{C}(13)$ & $111.3(3)$ \\
\hline $\mathrm{C}(19)-\mathrm{P}(1)-\mathrm{C}(2)$ & 113.2(3) \\
\hline $\mathrm{C}(7)-\mathrm{P}(1)-\mathrm{C}(2)$ & $106.4(3)$ \\
\hline $\mathrm{C}(13)-\mathrm{P}(1)-\mathrm{C}(2)$ & $106.4(3)$ \\
\hline $\mathrm{C}(49)-\mathrm{P}(2)-\mathrm{C}(43)$ & $114.6(3)$ \\
\hline $\mathrm{C}(49)-\mathrm{P}(2)-\mathrm{C}(32)$ & $112.1(3)$ \\
\hline $\mathrm{C}(43)-\mathrm{P}(2)-\mathrm{C}(32)$ & $109.5(3)$ \\
\hline
\end{tabular}




\begin{tabular}{|c|c|}
\hline $\mathrm{C}(49)-\mathrm{P}(2)-\mathrm{C}(37)$ & $107.9(3)$ \\
\hline $\mathrm{C}(43)-\mathrm{P}(2)-\mathrm{C}(37)$ & $107.3(3)$ \\
\hline $\mathrm{C}(32)-\mathrm{P}(2)-\mathrm{C}(37)$ & $104.9(3)$ \\
\hline $\mathrm{C}(20)-\mathrm{O}(2)-\mathrm{C}(21)$ & $115.1(6)$ \\
\hline $\mathrm{C}(23)-\mathrm{O}(4)-\mathrm{C}(24)$ & $114.7(6)$ \\
\hline $\mathrm{C}(50)-\mathrm{O}(6)-\mathrm{C}(51)$ & $116.4(5)$ \\
\hline $\mathrm{C}(53)-\mathrm{O}(8)-\mathrm{C}(54)$ & 114.1(6) \\
\hline $\mathrm{C}(22)-\mathrm{N}(1)-\mathrm{C}(25)$ & $122.0(6)$ \\
\hline $\mathrm{C}(22)-\mathrm{N}(1)-\mathrm{Pd}(1)$ & $122.0(5)$ \\
\hline $\mathrm{C}(25)-\mathrm{N}(1)-\mathrm{Pd}(1)$ & $116.0(4)$ \\
\hline $\mathrm{C}(52)-\mathrm{N}(2)-\mathrm{C}(55)$ & $122.6(5)$ \\
\hline $\mathrm{C}(52)-\mathrm{N}(2)-\mathrm{Pd}(2)$ & $129.8(4)$ \\
\hline $\mathrm{C}(55)-\mathrm{N}(2)-\mathrm{Pd}(2)$ & $107.5(4)$ \\
\hline $\mathrm{C}(2)-\mathrm{C}(1)-\mathrm{C}(6)$ & $118.7(6)$ \\
\hline $\mathrm{C}(2)-\mathrm{C}(1)-\mathrm{Pd}(1)$ & $119.4(5)$ \\
\hline $\mathrm{C}(6)-\mathrm{C}(1)-\mathrm{Pd}(1)$ & $121.8(5)$ \\
\hline $\mathrm{C}(1)-\mathrm{C}(2)-\mathrm{C}(3)$ & $121.7(6)$ \\
\hline $\mathrm{C}(1)-\mathrm{C}(2)-\mathrm{P}(1)$ & $118.0(5)$ \\
\hline $\mathrm{C}(3)-\mathrm{C}(2)-\mathrm{P}(1)$ & $120.0(5)$ \\
\hline$C(4)-C(3)-C(2)$ & $118.6(6)$ \\
\hline$C(3)-C(4)-C(5)$ & $119.6(7)$ \\
\hline$C(6)-C(5)-C(4)$ & $120.4(7)$ \\
\hline$C(5)-C(6)-C(1)$ & $120.8(6)$ \\
\hline $\mathrm{C}(8)-\mathrm{C}(7)-\mathrm{C}(12)$ & $120.1(7)$ \\
\hline $\mathrm{C}(8)-\mathrm{C}(7)-\mathrm{P}(1)$ & $120.8(5)$ \\
\hline $\mathrm{C}(12)-\mathrm{C}(7)-\mathrm{P}(1)$ & $119.0(5)$ \\
\hline $\mathrm{C}(9)-\mathrm{C}(8)-\mathrm{C}(7)$ & $120.2(7)$ \\
\hline $\mathrm{C}(8)-\mathrm{C}(9)-\mathrm{C}(10)$ & $120.4(7)$ \\
\hline $\mathrm{C}(9)-\mathrm{C}(10)-\mathrm{C}(11)$ & $120.0(8)$ \\
\hline$C(12)-C(11)-C(10)$ & 119.1(7) \\
\hline$C(7)-C(12)-C(11)$ & $120.1(7)$ \\
\hline$C(14)-C(13)-C(18)$ & $120.6(6)$ \\
\hline $\mathrm{C}(14)-\mathrm{C}(13)-\mathrm{P}(1)$ & $118.4(5)$ \\
\hline $\mathrm{C}(18)-\mathrm{C}(13)-\mathrm{P}(1)$ & 121.1(6) \\
\hline $\mathrm{C}(13)-\mathrm{C}(14)-\mathrm{C}(15)$ & $121.6(7)$ \\
\hline$C(14)-C(15)-C(16)$ & $118.8(8)$ \\
\hline$C(15)-C(16)-C(17)$ & $120.4(7)$ \\
\hline$C(18)-C(17)-C(16)$ & $119.3(7)$ \\
\hline $\mathrm{C}(17)-\mathrm{C}(18)-\mathrm{C}(13)$ & 119.2(8) \\
\hline$C(20)-C(19)-C(22)$ & $123.4(6)$ \\
\hline $\mathrm{C}(20)-\mathrm{C}(19)-\mathrm{P}(1)$ & $113.3(5)$ \\
\hline $\mathrm{C}(22)-\mathrm{C}(19)-\mathrm{P}(1)$ & $122.8(5)$ \\
\hline $\mathrm{O}(1)-\mathrm{C}(20)-\mathrm{O}(2)$ & $122.3(6)$ \\
\hline $\mathrm{O}(1)-\mathrm{C}(20)-\mathrm{C}(19)$ & $124.7(6)$ \\
\hline $\mathrm{O}(2)-\mathrm{C}(20)-\mathrm{C}(19)$ & $112.9(6)$ \\
\hline $\mathrm{N}(1)-\mathrm{C}(22)-\mathrm{C}(19)$ & $123.3(6)$ \\
\hline $\mathrm{N}(1)-\mathrm{C}(22)-\mathrm{C}(23)$ & $120.9(6)$ \\
\hline $\mathrm{C}(19)-\mathrm{C}(22)-\mathrm{C}(23)$ & $115.8(5)$ \\
\hline $\mathrm{O}(3)-\mathrm{C}(23)-\mathrm{O}(4)$ & $124.4(6)$ \\
\hline $\mathrm{O}(3)-\mathrm{C}(23)-\mathrm{C}(22)$ & $124.0(6)$ \\
\hline $\mathrm{O}(4)-\mathrm{C}(23)-\mathrm{C}(22)$ & $111.6(6)$ \\
\hline$C(26)-C(25)-C(30)$ & $120.6(7)$ \\
\hline $\mathrm{C}(26)-\mathrm{C}(25)-\mathrm{N}(1)$ & $120.5(6)$ \\
\hline $\mathrm{C}(30)-\mathrm{C}(25)-\mathrm{N}(1)$ & $118.9(6)$ \\
\hline$C(25)-C(26)-C(27)$ & $120.0(7)$ \\
\hline $\mathrm{C}(26)-\mathrm{C}(27)-\mathrm{C}(28)$ & $119.4(7)$ \\
\hline $\mathrm{C}(27)-\mathrm{C}(28)-\mathrm{C}(29)$ & $119.5(7)$ \\
\hline $\mathrm{C}(30)-\mathrm{C}(29)-\mathrm{C}(28)$ & $120.5(7)$ \\
\hline
\end{tabular}




\begin{tabular}{|c|c|}
\hline$C(29)-C(30)-C(25)$ & $120.0(7)$ \\
\hline$C(32)-C(31)-C(36)$ & $117.6(6)$ \\
\hline $\mathrm{C}(32)-\mathrm{C}(31)-\mathrm{Pd}(2)$ & $119.4(5)$ \\
\hline$C(36)-C(31)-P d(2)$ & $122.9(5)$ \\
\hline $\mathrm{C}(33)-\mathrm{C}(32)-\mathrm{C}(31)$ & $120.6(6)$ \\
\hline $\mathrm{C}(33)-\mathrm{C}(32)-\mathrm{P}(2)$ & $122.1(5)$ \\
\hline $\mathrm{C}(31)-\mathrm{C}(32)-\mathrm{P}(2)$ & $117.2(5)$ \\
\hline$C(34)-C(33)-C(32)$ & $120.1(7)$ \\
\hline $\mathrm{C}(33)-\mathrm{C}(34)-\mathrm{C}(35)$ & $120.0(7)$ \\
\hline$C(34)-C(35)-C(36)$ & $121.8(7)$ \\
\hline$C(35)-C(36)-C(31)$ & $119.8(7)$ \\
\hline $\mathrm{C}(42)-\mathrm{C}(37)-\mathrm{C}(38)$ & $118.4(6)$ \\
\hline $\mathrm{C}(42)-\mathrm{C}(37)-\mathrm{P}(2)$ & $122.2(6)$ \\
\hline $\mathrm{C}(38)-\mathrm{C}(37)-\mathrm{P}(2)$ & $119.3(5)$ \\
\hline $\mathrm{C}(39)-\mathrm{C}(38)-\mathrm{C}(37)$ & $120.3(8)$ \\
\hline $\mathrm{C}(40)-\mathrm{C}(39)-\mathrm{C}(38)$ & $120.3(8)$ \\
\hline $\mathrm{C}(41)-\mathrm{C}(40)-\mathrm{C}(39)$ & $119.4(7)$ \\
\hline$C(40)-C(41)-C(42)$ & $121.1(8)$ \\
\hline $\mathrm{C}(37)-\mathrm{C}(42)-\mathrm{C}(41)$ & $120.4(7)$ \\
\hline $\mathrm{C}(48)-\mathrm{C}(43)-\mathrm{C}(44)$ & $119.6(6)$ \\
\hline $\mathrm{C}(48)-\mathrm{C}(43)-\mathrm{P}(2)$ & $119.2(5)$ \\
\hline $\mathrm{C}(44)-\mathrm{C}(43)-\mathrm{P}(2)$ & $121.2(5)$ \\
\hline$C(45)-C(44)-C(43)$ & $119.9(6)$ \\
\hline$C(46)-C(45)-C(44)$ & $120.0(6)$ \\
\hline$C(45)-C(46)-C(47)$ & $120.0(7)$ \\
\hline $\mathrm{C}(48)-\mathrm{C}(47)-\mathrm{C}(46)$ & $119.9(7)$ \\
\hline$C(47)-C(48)-C(43)$ & $120.5(6)$ \\
\hline$C(52)-C(49)-C(50)$ & $117.6(5)$ \\
\hline $\mathrm{C}(52)-\mathrm{C}(49)-\mathrm{P}(2)$ & $122.1(5)$ \\
\hline $\mathrm{C}(50)-\mathrm{C}(49)-\mathrm{P}(2)$ & $120.3(5)$ \\
\hline $\mathrm{O}(5)-\mathrm{C}(50)-\mathrm{O}(6)$ & $121.5(6)$ \\
\hline $\mathrm{O}(5)-\mathrm{C}(50)-\mathrm{C}(49)$ & $127.3(6)$ \\
\hline $\mathrm{O}(6)-\mathrm{C}(50)-\mathrm{C}(49)$ & $111.1(5)$ \\
\hline $\mathrm{N}(2)-\mathrm{C}(52)-\mathrm{C}(49)$ & $124.9(5)$ \\
\hline$N(2)-C(52)-C(53)$ & $116.5(5)$ \\
\hline$C(49)-C(52)-C(53)$ & $118.5(6)$ \\
\hline $\mathrm{O}(7)-\mathrm{C}(53)-\mathrm{O}(8)$ & $126.4(6)$ \\
\hline $\mathrm{O}(7)-\mathrm{C}(53)-\mathrm{C}(52)$ & $124.5(6)$ \\
\hline $\mathrm{O}(8)-\mathrm{C}(53)-\mathrm{C}(52)$ & $108.9(6)$ \\
\hline$C(60)-C(55)-C(56)$ & $120.9(6)$ \\
\hline $\mathrm{C}(60)-\mathrm{C}(55)-\mathrm{N}(2)$ & $119.9(6)$ \\
\hline $\mathrm{C}(56)-\mathrm{C}(55)-\mathrm{N}(2)$ & $119.0(6)$ \\
\hline$C(55)-C(56)-C(57)$ & $118.7(7)$ \\
\hline$C(58)-C(57)-C(56)$ & $121.1(7)$ \\
\hline $\mathrm{C}(57)-\mathrm{C}(58)-\mathrm{C}(59)$ & $118.3(7)$ \\
\hline$C(60)-C(59)-C(58)$ & $121.5(7)$ \\
\hline$C(59)-C(60)-C(55)$ & $119.4(8)$ \\
\hline $\mathrm{Cl}(3)-\mathrm{C}(100)-\mathrm{Cl}(5)$ & $111.4(8)$ \\
\hline $\mathrm{Cl}(3)-\mathrm{C}(100)-\mathrm{Cl}(4)$ & $107.0(8)$ \\
\hline $\mathrm{Cl}(5)-\mathrm{C}(100)-\mathrm{Cl}(4)$ & $109.3(7)$ \\
\hline
\end{tabular}


Table S14. Anisotropic displacement parameters $\left(\AA^{2} \times 10^{3}\right)$ for $100.5 \mathrm{CHCl}_{3}$. The anisotropic displacement factor exponent takes the form: $-2 \pi^{2}\left[h^{2} a^{* 2} U^{11}+\ldots+2 h k a^{*} b^{*} U^{12}\right]$

\begin{tabular}{|c|c|c|c|c|c|c|}
\hline & $\mathrm{U}^{11}$ & $\mathrm{U}^{22}$ & $\mathrm{U}^{33}$ & $\mathrm{U}^{23}$ & $\mathrm{U}^{13}$ & $\mathrm{U}^{12}$ \\
\hline $\operatorname{Pd}(1)$ & $32(1)$ & $15(1)$ & $26(1)$ & $1(1)$ & $7(1)$ & $1(1)$ \\
\hline $\operatorname{Pd}(2)$ & $30(1)$ & $18(1)$ & $27(1)$ & $0(1)$ & $9(1)$ & $2(1)$ \\
\hline $\mathrm{Cl}(2)$ & $41(1)$ & $21(1)$ & $26(1)$ & $-1(1)$ & $5(1)$ & $7(1)$ \\
\hline $\mathrm{Cl}(1)$ & $39(1)$ & $20(1)$ & $28(1)$ & $-1(1)$ & $8(1)$ & $5(1)$ \\
\hline $\mathrm{P}(1)$ & $34(1)$ & $16(1)$ & $38(1)$ & $2(1)$ & 11(1) & $1(1)$ \\
\hline $\mathrm{P}(2)$ & $29(1)$ & $22(1)$ & $27(1)$ & $2(1)$ & $7(1)$ & $2(1)$ \\
\hline $\mathrm{O}(1)$ & $32(3)$ & $25(3)$ & $48(3)$ & $4(2)$ & $12(2)$ & $-1(2)$ \\
\hline $\mathrm{O}(2)$ & $38(3)$ & $29(3)$ & $40(3)$ & $0(2)$ & $18(2)$ & $-1(2)$ \\
\hline $\mathrm{O}(3)$ & $40(3)$ & $37(3)$ & $38(3)$ & $11(2)$ & $11(2)$ & $10(2)$ \\
\hline $\mathrm{O}(4)$ & $47(3)$ & $29(3)$ & $32(3)$ & $-1(2)$ & $13(2)$ & $0(2)$ \\
\hline $\mathrm{O}(5)$ & $37(3)$ & $26(3)$ & $52(3)$ & $-3(2)$ & $14(2)$ & $8(2)$ \\
\hline $\mathrm{O}(6)$ & $33(3)$ & $23(3)$ & $44(3)$ & $1(2)$ & $8(2)$ & $-2(2)$ \\
\hline $\mathrm{O}(7)$ & $32(3)$ & $44(3)$ & $41(3)$ & $-6(2)$ & $-5(2)$ & $16(2)$ \\
\hline $\mathrm{O}(8)$ & $40(3)$ & $36(3)$ & $30(3)$ & $0(2)$ & $8(2)$ & $10(2)$ \\
\hline $\mathrm{N}(1)$ & $34(3)$ & $17(3)$ & $29(3)$ & $0(2)$ & $8(2)$ & $0(2)$ \\
\hline $\mathrm{N}(2)$ & $30(3)$ & $24(3)$ & $23(3)$ & $0(2)$ & $8(2)$ & $7(2)$ \\
\hline $\mathrm{C}(1)$ & $36(4)$ & $21(4)$ & $24(3)$ & 1(3) & $-1(3)$ & $3(3)$ \\
\hline$C(2)$ & $33(4)$ & $19(3)$ & $35(4)$ & $-2(3)$ & $9(3)$ & $-1(3)$ \\
\hline$C(3)$ & $43(4)$ & $19(4)$ & $60(5)$ & $-2(3)$ & $19(4)$ & $5(3)$ \\
\hline $\mathrm{C}(4)$ & $44(5)$ & $29(4)$ & $74(6)$ & 2(4) & $17(4)$ & $6(3)$ \\
\hline$C(5)$ & $56(5)$ & $29(4)$ & $59(5)$ & $7(4)$ & $30(4)$ & $2(4)$ \\
\hline$C(6)$ & $35(4)$ & $25(4)$ & $42(4)$ & $4(3)$ & $10(3)$ & $2(3)$ \\
\hline$C(7)$ & $30(4)$ & $20(3)$ & $37(4)$ & $2(3)$ & $5(3)$ & $-5(3)$ \\
\hline $\mathrm{C}(8)$ & $46(4)$ & $24(4)$ & $41(4)$ & $3(3)$ & $4(3)$ & $-1(3)$ \\
\hline $\mathrm{C}(9)$ & $60(5)$ & $26(4)$ & $38(4)$ & $-5(3)$ & $6(4)$ & $-12(4)$ \\
\hline$C(10)$ & $48(5)$ & $43(5)$ & $43(5)$ & $5(4)$ & $-6(4)$ & $-6(4)$ \\
\hline $\mathrm{C}(11)$ & $52(5)$ & $33(4)$ & $53(5)$ & 2(4) & $6(4)$ & $3(4)$ \\
\hline$C(12)$ & $37(4)$ & $25(4)$ & $42(4)$ & $0(3)$ & $0(3)$ & $0(3)$ \\
\hline $\mathrm{C}(13)$ & $39(4)$ & $14(3)$ & $39(4)$ & $1(3)$ & $15(3)$ & $-1(3)$ \\
\hline $\mathrm{C}(14)$ & $37(4)$ & $20(4)$ & $49(4)$ & $5(3)$ & $4(3)$ & $1(3)$ \\
\hline$C(15)$ & $38(4)$ & $31(4)$ & $62(5)$ & $11(4)$ & $6(4)$ & $6(3)$ \\
\hline$C(16)$ & $67(6)$ & $19(4)$ & $65(6)$ & $6(4)$ & $30(5)$ & $7(4)$ \\
\hline$C(17)$ & $54(5)$ & $23(4)$ & $55(5)$ & $3(3)$ & $12(4)$ & $-14(3)$ \\
\hline $\mathrm{C}(18)$ & $46(5)$ & $25(4)$ & $47(4)$ & $3(3)$ & $17(4)$ & $-1(3)$ \\
\hline C(19) & $38(4)$ & $20(3)$ & $31(4)$ & $7(3)$ & $7(3)$ & $0(3)$ \\
\hline$C(20)$ & $38(4)$ & $20(4)$ & $39(4)$ & $8(3)$ & $12(3)$ & $7(3)$ \\
\hline $\mathrm{C}(21)$ & $40(5)$ & $41(5)$ & $49(5)$ & $10(3)$ & $20(4)$ & $8(3)$ \\
\hline $\mathrm{C}(22)$ & $35(4)$ & $20(3)$ & $32(4)$ & $2(3)$ & $13(3)$ & $11(3)$ \\
\hline $\mathrm{C}(23)$ & $33(4)$ & $28(4)$ & $32(4)$ & $5(3)$ & $13(3)$ & $1(3)$ \\
\hline $\mathrm{C}(24)$ & $85(6)$ & $37(5)$ & $25(4)$ & $-1(3)$ & $13(4)$ & $-5(4)$ \\
\hline$C(25)$ & $38(4)$ & $34(4)$ & $21(3)$ & $-9(3)$ & $8(3)$ & $1(3)$ \\
\hline$C(26)$ & $34(4)$ & $28(4)$ & $32(4)$ & 1(3) & 11(3) & $5(3)$ \\
\hline $\mathrm{C}(27)$ & $35(4)$ & $49(5)$ & $26(4)$ & $-3(3)$ & $3(3)$ & $0(3)$ \\
\hline $\mathrm{C}(28)$ & $47(5)$ & $38(5)$ & $32(4)$ & $-9(3)$ & $3(3)$ & $-8(4)$ \\
\hline C(29) & $53(5)$ & $27(4)$ & $37(4)$ & $-10(3)$ & $8(4)$ & $-2(4)$ \\
\hline $\mathrm{C}(30)$ & $34(4)$ & $21(4)$ & $34(4)$ & $-1(3)$ & $9(3)$ & $-1(3)$ \\
\hline $\mathrm{C}(31)$ & $25(4)$ & $29(4)$ & $30(4)$ & $-2(3)$ & $1(3)$ & $5(3)$ \\
\hline $\mathrm{C}(32)$ & $30(4)$ & $25(4)$ & $28(4)$ & $0(3)$ & $8(3)$ & $4(3)$ \\
\hline $\mathrm{C}(33)$ & $50(5)$ & $28(4)$ & $33(4)$ & $6(3)$ & $5(3)$ & $7(3)$ \\
\hline $\mathrm{C}(34)$ & $52(5)$ & $37(4)$ & $29(4)$ & $-3(3)$ & $-6(3)$ & $4(4)$ \\
\hline$C(35)$ & $33(4)$ & $34(4)$ & $41(4)$ & $-13(3)$ & $-1(3)$ & $5(3)$ \\
\hline$C(36)$ & $36(4)$ & $25(4)$ & $37(4)$ & $-7(3)$ & $4(3)$ & $-2(3)$ \\
\hline
\end{tabular}




\begin{tabular}{lcccccc}
$\mathrm{C}(37)$ & $40(4)$ & $27(4)$ & $27(4)$ & $4(3)$ & $8(3)$ & $1(3)$ \\
$\mathrm{C}(38)$ & $52(5)$ & $32(4)$ & $49(5)$ & $13(3)$ & $7(4)$ & $4(4)$ \\
$\mathrm{C}(39)$ & $77(7)$ & $47(5)$ & $54(5)$ & $23(4)$ & $-9(5)$ & $8(5)$ \\
$\mathrm{C}(40)$ & $71(6)$ & $33(5)$ & $47(5)$ & $9(4)$ & $3(4)$ & $-14(4)$ \\
$\mathrm{C}(41)$ & $55(5)$ & $38(5)$ & $55(5)$ & $1(4)$ & $18(4)$ & $-14(4)$ \\
$\mathrm{C}(42)$ & $43(4)$ & $30(4)$ & $33(4)$ & $0(3)$ & $9(3)$ & $-8(3)$ \\
$\mathrm{C}(43)$ & $27(3)$ & $20(3)$ & $24(3)$ & $3(3)$ & $8(3)$ & $-1(3)$ \\
$\mathrm{C}(44)$ & $32(4)$ & $38(4)$ & $30(4)$ & $-6(3)$ & $6(3)$ & $-2(3)$ \\
$\mathrm{C}(45)$ & $29(4)$ & $40(4)$ & $50(5)$ & $-4(3)$ & $9(3)$ & $11(3)$ \\
$\mathrm{C}(46)$ & $34(4)$ & $31(4)$ & $62(5)$ & $-7(4)$ & $-7(4)$ & $7(3)$ \\
$\mathrm{C}(47)$ & $44(4)$ & $32(4)$ & $37(4)$ & $2(3)$ & $-1(3)$ & $10(3)$ \\
$\mathrm{C}(48)$ & $38(4)$ & $22(4)$ & $35(4)$ & $1(3)$ & $6(3)$ & $4(3)$ \\
$\mathrm{C}(49)$ & $26(3)$ & $20(3)$ & $33(4)$ & $6(3)$ & $6(3)$ & $8(3)$ \\
$\mathrm{C}(50)$ & $36(4)$ & $25(4)$ & $32(4)$ & $3(3)$ & $3(3)$ & $2(3)$ \\
$\mathrm{C}(51)$ & $44(4)$ & $15(4)$ & $66(5)$ & $4(3)$ & $10(4)$ & $-4(3)$ \\
$\mathrm{C}(52)$ & $26(4)$ & $27(4)$ & $23(3)$ & $-2(3)$ & $4(3)$ & $1(3)$ \\
$\mathrm{C}(53)$ & $35(4)$ & $31(4)$ & $31(4)$ & $0(3)$ & $13(3)$ & $-2(3)$ \\
$\mathrm{C}(54)$ & $41(4)$ & $46(5)$ & $41(4)$ & $7(3)$ & $26(3)$ & $17(4)$ \\
$\mathrm{C}(55)$ & $29(4)$ & $24(4)$ & $35(4)$ & $0(3)$ & $10(3)$ & $2(3)$ \\
$\mathrm{C}(56)$ & $37(4)$ & $24(4)$ & $45(4)$ & $3(3)$ & $6(3)$ & $12(3)$ \\
$\mathrm{C}(57)$ & $26(4)$ & $33(4)$ & $56(5)$ & $0(3)$ & $-6(3)$ & $2(3)$ \\
$\mathrm{C}(58)$ & $35(4)$ & $42(5)$ & $58(5)$ & $7(4)$ & $14(4)$ & $-3(4)$ \\
$\mathrm{C}(59)$ & $41(5)$ & $51(5)$ & $45(5)$ & $12(4)$ & $19(4)$ & $0(4)$ \\
$\mathrm{C}(60)$ & $30(4)$ & $41(4)$ & $41(4)$ & $4(3)$ & $11(3)$ & $-3(3)$ \\
$\mathrm{C}(100)$ & $40(9)$ & $34(10)$ & $114(16)$ & $-16(10)$ & $2(10)$ & $7(7)$ \\
$\mathrm{Cl}(3)$ & $78(4)$ & $73(4)$ & $138(5)$ & $-53(4)$ & $-20(3)$ & $12(3)$ \\
$\mathrm{Cl}(4)$ & $53(2)$ & $50(3)$ & $70(3)$ & $11(2)$ & $3(2)$ & $23(2)$ \\
$\mathrm{Cl}(5)$ & $118(5)$ & $52(3)$ & $113(5)$ & $-10(3)$ & $44(4)$ & $-31(3)$ \\
& & & & & \\
\hline & & & & & \\
\end{tabular}


Table S15. Hydrogen coordinates ( x 10 $)$ and isotropic displacement parameters $\left(\AA^{2} \times 10^{3}\right)$ for $100.5 \mathrm{CHCl}_{3}$.

\begin{tabular}{|c|c|c|c|c|}
\hline & $\mathrm{x}$ & $\mathrm{y}$ & $\mathrm{z}$ & $\mathrm{U}(\mathrm{eq})$ \\
\hline $\mathrm{H}(3 \mathrm{~A})$ & 5010 & 2374 & 3571 & 49 \\
\hline $\mathrm{H}(4 \mathrm{~A})$ & 6726 & 2465 & 2882 & 58 \\
\hline $\mathrm{H}(5 \mathrm{~A})$ & 7226 & 3129 & 2377 & 57 \\
\hline $\mathrm{H}(6 \mathrm{~A})$ & 6042 & 3687 & 2568 & 41 \\
\hline $\mathrm{H}(8 \mathrm{~A})$ & 2820 & 2655 & 2279 & 44 \\
\hline $\mathrm{H}(9 \mathrm{~A})$ & 1535 & 2813 & 1217 & 50 \\
\hline $\mathrm{H}(10 \mathrm{~A})$ & 89 & 3284 & 1471 & 54 \\
\hline $\mathrm{H}(11 \mathrm{~A})$ & -20 & 3623 & 2795 & 55 \\
\hline $\mathrm{H}(12 \mathrm{~A})$ & 1280 & 3455 & 3868 & 41 \\
\hline $\mathrm{H}(14 \mathrm{~A})$ & 4126 & 2474 & 5337 & 43 \\
\hline $\mathrm{H}(15 \mathrm{~A})$ & 4057 & 1795 & 5847 & 52 \\
\hline $\mathrm{H}(16 \mathrm{~A})$ & 2990 & 1295 & 5099 & 60 \\
\hline $\mathrm{H}(17 \mathrm{~A})$ & 1944 & 1490 & 3878 & 53 \\
\hline $\mathrm{H}(18 \mathrm{~A})$ & 1998 & 2183 & 3409 & 47 \\
\hline $\mathrm{H}(21 \mathrm{~A})$ & 972 & 3401 & 7165 & 65 \\
\hline $\mathrm{H}(21 \mathrm{~B})$ & 905 & 2940 & 6787 & 65 \\
\hline $\mathrm{H}(21 \mathrm{C})$ & 316 & 3316 & 6288 & 65 \\
\hline $\mathrm{H}(24 \mathrm{~A})$ & 3537 & 4116 & 7690 & 74 \\
\hline $\mathrm{H}(24 \mathrm{~B})$ & 4712 & 3876 & 7626 & 74 \\
\hline $\mathrm{H}(24 \mathrm{C})$ & 3565 & 3618 & 7693 & 74 \\
\hline$H(26 A)$ & 6166 & 3483 & 5493 & 38 \\
\hline $\mathrm{H}(27 \mathrm{~A})$ & 7763 & 3846 & 5948 & 44 \\
\hline $\mathrm{H}(28 \mathrm{~A})$ & 7865 & 4567 & 5730 & 47 \\
\hline $\mathrm{H}(29 \mathrm{~A})$ & 6412 & 4906 & 4984 & 47 \\
\hline $\mathrm{H}(30 \mathrm{~A})$ & 4854 & 4542 & 4536 & 36 \\
\hline $\mathrm{H}(33 \mathrm{~A})$ & 1961 & 5263 & -383 & 44 \\
\hline $\mathrm{H}(34 \mathrm{~A})$ & 1146 & 4733 & -1171 & 47 \\
\hline $\mathrm{H}(35 \mathrm{~A})$ & 742 & 4103 & -536 & 43 \\
\hline $\mathrm{H}(36 \mathrm{~A})$ & 1125 & 3986 & 890 & 39 \\
\hline $\mathrm{H}(38 \mathrm{~A})$ & 1381 & 5986 & 577 & 53 \\
\hline $\mathrm{H}(39 \mathrm{~A})$ & 1763 & 6506 & -409 & 71 \\
\hline $\mathrm{H}(40 \mathrm{~A})$ & 3554 & 6572 & -968 & 60 \\
\hline $\mathrm{H}(41 \mathrm{~A})$ & 4970 & 6141 & -495 & 59 \\
\hline $\mathrm{H}(42 \mathrm{~A})$ & 4629 & 5638 & 530 & 43 \\
\hline $\mathrm{H}(44 \mathrm{~A})$ & 4453 & 4828 & 800 & 40 \\
\hline $\mathrm{H}(45 \mathrm{~A})$ & 6196 & 4628 & 1369 & 48 \\
\hline $\mathrm{H}(46 \mathrm{~A})$ & 6682 & 4815 & 2755 & 51 \\
\hline $\mathrm{H}(47 \mathrm{~A})$ & 5452 & 5221 & 3568 & 45 \\
\hline $\mathrm{H}(48 \mathrm{~A})$ & 3771 & 5451 & 2977 & 38 \\
\hline $\mathrm{H}(51 \mathrm{~A})$ & 3712 & 6658 & 2402 & 63 \\
\hline $\mathrm{H}(51 \mathrm{~B})$ & 2484 & 6680 & 2811 & 63 \\
\hline $\mathrm{H}(51 \mathrm{C})$ & 2632 & 6721 & 1802 & 63 \\
\hline $\mathrm{H}(54 \mathrm{~A})$ & -933 & 5782 & 4714 & 64 \\
\hline $\mathrm{H}(54 \mathrm{~B})$ & -1675 & 5462 & 4169 & 64 \\
\hline $\mathrm{H}(54 \mathrm{C})$ & -1437 & 5918 & 3807 & 64 \\
\hline $\mathrm{H}(56 \mathrm{~A})$ & -734 & 4557 & 1657 & 42 \\
\hline $\mathrm{H}(57 \mathrm{~A})$ & -2192 & 4118 & 2109 & 46 \\
\hline $\mathrm{H}(58 \mathrm{~A})$ & -2354 & 3937 & 3539 & 54 \\
\hline $\mathrm{H}(59 \mathrm{~A})$ & -1118 & 4243 & 4541 & 55 \\
\hline $\mathrm{H}(60 \mathrm{~A})$ & 331 & 4669 & 4109 & 45 \\
\hline
\end{tabular}


Table S16. Calculated cartesian coordinates and energy for ligand L1.

\begin{tabular}{|c|c|c|c|}
\hline \multicolumn{4}{|c|}{ Energy $=-1855.57971899$} \\
\hline $\mathrm{P}$ & 1.093995 & -0.281155 & 0.051162 \\
\hline $\mathrm{C}$ & -1.252554 & 0.927947 & -0.544668 \\
\hline $\mathrm{C}$ & 0.180153 & 1.117090 & -0.500528 \\
\hline $\mathrm{C}$ & 0.750314 & 2.356367 & -0.994059 \\
\hline $\mathrm{C}$ & -2.138186 & 2.168206 & -0.657515 \\
\hline $\mathrm{N}$ & -1.746416 & -0.272596 & -0.515689 \\
\hline $\mathrm{O}$ & -2.094586 & 2.891207 & 0.483575 \\
\hline $\mathrm{O}$ & -2.835311 & 2.420230 & -1.610811 \\
\hline $\mathrm{O}$ & 2.111821 & 2.326839 & -1.115674 \\
\hline $\mathrm{O}$ & 0.108842 & 3.351968 & -1.307503 \\
\hline $\mathrm{H}$ & -6.846835 & -1.641461 & 0.075757 \\
\hline $\mathrm{C}$ & 3.054580 & 0.469058 & 1.936251 \\
\hline $\mathrm{H}$ & 2.245285 & 0.493545 & 2.656014 \\
\hline 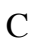 & 4.352962 & 0.783478 & 2.354057 \\
\hline $\mathrm{H}$ & 4.531542 & 1.053853 & 3.389674 \\
\hline $\mathrm{C}$ & 5.413708 & 0.742326 & 1.444366 \\
\hline $\mathrm{H}$ & 6.421021 & 0.981278 & 298 \\
\hline $\mathrm{C}$ & 5.172006 & 0.389356 & 0.110519 \\
\hline $\mathrm{H}$ & 5.990451 & 0.356588 & -0.601439 \\
\hline $\mathrm{C}$ & 3.876684 & 0.080222 & -0.310964 \\
\hline $\mathrm{H}$ & 3.699115 & -0.175943 & -1.348198 \\
\hline $\mathrm{C}$ & 0.286755 & -0.988109 & 1.537337 \\
\hline$\pi$ & 0.314944 & -2.364463 & 1.816220 \\
\hline 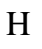 & 0.754261 & -3.058021 & 1.109464 \\
\hline $\mathrm{C}$ & -0.250835 & -2.856514 & 2.997707 \\
\hline П & -0.230613 & -3.923031 & 3.197260 \\
\hline $\mathrm{C}$ & -0.850072 & -1.982334 & 3.910015 \\
\hline $\mathrm{H}$ & -1.292178 & -2.366950 & 4.823402 \\
\hline 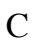 & -0.890463 & -0.610338 & 3.633310 \\
\hline $\mathrm{H}$ & -1.369131 & 0.072140 & 4.328066 \\
\hline$C$ & -0.327720 & -0.112699 & 2.454609 \\
\hline $\mathrm{H}$ & -0.390898 & 0.946681 & 2.228352 \\
\hline $\mathrm{C}$ & 1.315468 & -1.610263 & -1.191682 \\
\hline 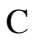 & 2.242303 & -2.655881 & -1.004548 \\
\hline $\mathrm{H}$ & 2.863313 & -2.684153 & -0.115289 \\
\hline $\mathrm{C}$ & 2.386235 & -3.653000 & -1.974653 \\
\hline $\mathrm{H}$ & 3.102422 & -4.453922 & -1.821718 \\
\hline $\mathrm{C}$ & 1.614607 & -3.611817 & -3.142387 \\
\hline $\mathrm{H}$ & 1.729754 & -4.384304 & -3.896078 \\
\hline $\mathrm{C}$ & 0.700212 & -2.570949 & -3.339165 \\
\hline $\mathrm{H}$ & 0.104606 & -2.532485 & -4.245181 \\
\hline $\mathrm{C}$ & 0.548340 & -1.573603 & -2.369818 \\
\hline $\mathrm{H}$ & -0.167846 & -0.774780 & -2.514028 \\
\hline $\mathrm{C}$ & -5.809897 & -1.343115 & -0.042207 \\
\hline $\mathrm{C}$ & -2.813067 & 4.157246 & 0.416344 \\
\hline $\mathrm{H}$ & -2.710224 & 4.601361 & 1.406620 \\
\hline $\mathrm{H}$ & -2.353797 & 4.791849 & -0.344468 \\
\hline $\mathrm{H}$ & -3.863712 & 3.990053 & 0.169609 \\
\hline $\mathrm{C}$ & 2.710158 & 3.567222 & -1.570106 \\
\hline $\mathrm{H}$ & 3.784100 & 3.376073 & -1.587437 \\
\hline $\mathrm{H}$ & 2.346671 & 3.829182 & -2.566687 \\
\hline $\mathrm{H}$ & 2.475843 & 4.385053 & -0.884302 \\
\hline $\mathrm{C}$ & -5.270604 & -0.329383 & 0.757444 \\
\hline $\mathrm{H}$ & -5.889518 & 0.158921 & 1.504808 \\
\hline
\end{tabular}




$\begin{array}{lrrr}\mathrm{C} & -3.936332 & 0.060331 & 0.609237 \\ \mathrm{H} & -3.520250 & 0.832183 & 1.246633 \\ \mathrm{C} & -3.114385 & -0.560256 & -0.355038 \\ \mathrm{C} & -3.658743 & -1.601532 & -1.134867 \\ \mathrm{H} & -3.015348 & -2.096788 & -1.854277 \\ \mathrm{C} & -4.993937 & -1.977709 & -0.989216 \\ \mathrm{H} & -5.399131 & -2.771591 & -1.609791 \\ \mathrm{C} & 2.802358 & 0.116150 & 0.598775\end{array}$


Table S17. Calculated cartesian coordinates and energy for complex 9.

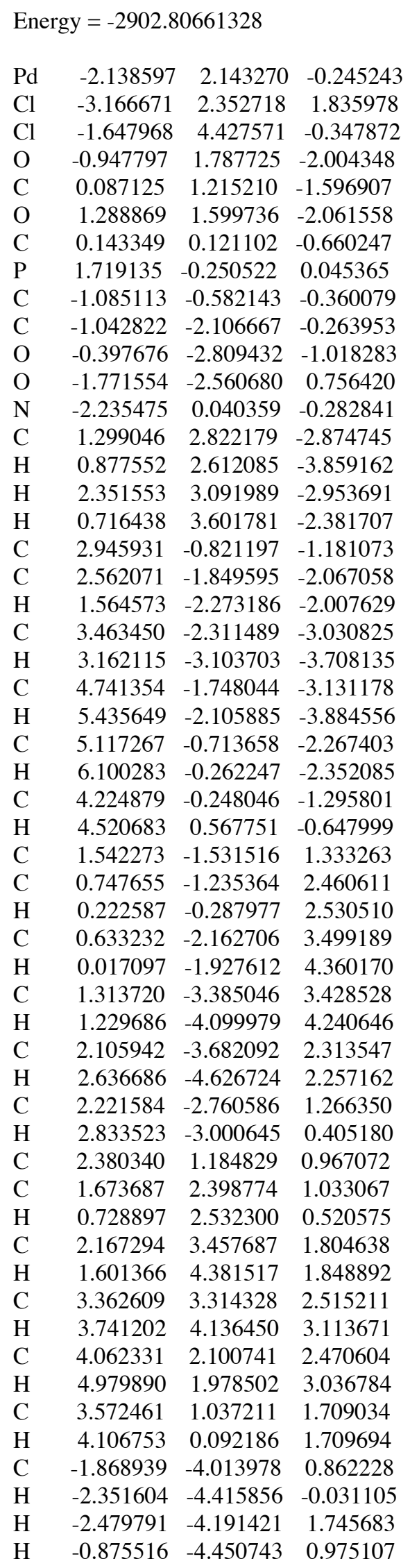




$\begin{array}{lrrr}\mathrm{C} & -3.472146 & -0.640965 & -0.507308 \\ \mathrm{C} & -4.545714 & -0.470897 & 0.382198 \\ \mathrm{H} & -4.420489 & 0.179535 & 1.238821 \\ \mathrm{C} & -5.751377 & -1.129988 & 0.136281 \\ \mathrm{H} & -6.577275 & -1.000610 & 0.828155 \\ \mathrm{C} & -5.905993 & -1.943320 & -0.995432 \\ \mathrm{H} & -6.851000 & -2.442960 & -1.182844 \\ \mathrm{C} & -4.843469 & -2.090050 & -1.893663 \\ \mathrm{H} & -4.961517 & -2.695783 & -2.786571 \\ \mathrm{C} & -3.629147 & -1.438244 & -1.657076 \\ \mathrm{H} & -2.816475 & -1.514843 & -2.371903\end{array}$


Table S18. Calculated cartesian coordinates and energy for complex 9co.

\begin{tabular}{|c|c|c|c|}
\hline \multicolumn{4}{|c|}{ Energy $=-2902.79212933$} \\
\hline $\mathrm{Cl}$ & 1.975647 & 2.884638 & 1.101213 \\
\hline $\mathrm{Pd}$ & -0.132328 & 2.653695 & 0.165334 \\
\hline $\mathrm{Cl}$ & -0.728986 & 4.782059 & 0.926410 \\
\hline $\mathrm{C}$ & 0.105114 & 0.564166 & -0.710045 \\
\hline $\mathrm{C}$ & -1.344824 & 0.210954 & -0.662627 \\
\hline $\mathrm{C}$ & -2.233808 & 1.322518 & -1.184722 \\
\hline $\mathrm{O}$ & -2.024912 & 2.488789 & -0.836110 \\
\hline $\mathrm{O}$ & -3.214883 & 0.960684 & -1.975970 \\
\hline $\mathrm{N}$ & -1.768572 & -0.878351 & -0.137104 \\
\hline $\mathrm{P}$ & 1.129891 & -0.685724 & 0.152754 \\
\hline $\mathrm{C}$ & 0.526656 & 1.029603 & -2.064188 \\
\hline $\mathrm{O}$ & -0.268085 & 1.303275 & -2.954291 \\
\hline $\mathrm{O}$ & 1.858412 & 1.124231 & -2.211828 \\
\hline $\mathrm{C}$ & -4.053445 & 2.051538 & -2.492803 \\
\hline $\mathrm{H}$ & -4.776285 & 1.561136 & -3.141564 \\
\hline $\mathrm{H}$ & -3.430896 & 2.754484 & -3.047658 \\
\hline $\mathrm{H}$ & -4.546269 & 2.561468 & -1.6 \\
\hline $\mathrm{C}$ & -3.124040 & -1.138327 & 0.158841 \\
\hline $\mathrm{C}$ & -3.656786 & -2.388589 & -0.205169 \\
\hline $\mathrm{H}$ & -3.027584 & -3.088990 & -0.743651 \\
\hline $\mathrm{C}$ & -4.977950 & -2.706431 & 0.113032 \\
\hline $\mathrm{H}$ & -5.386044 & -3.666334 & 7646 \\
\hline $\mathrm{C}$ & -5.772599 & -1.800186 & 704 \\
\hline $\mathrm{H}$ & -6.795115 & -2.055918 & 1.086318 \\
\hline $\mathrm{C}$ & -5.233181 & -0.570813 & 1.224604 \\
\hline $\mathrm{H}$ & -5.833796 & 0.128324 & 1.797934 \\
\hline $\mathrm{C}$ & -3.917037 & -0.235631 & 0.894075 \\
\hline $\mathrm{H}$ & -3.491223 & 0.704729 & 279 \\
\hline $\mathrm{C}$ & 2.310829 & 1.740942 & -3.454569 \\
\hline $\mathrm{H}$ & 3.398394 & 1.703252 & -3.406074 \\
\hline $\mathrm{H}$ & 1.964416 & 2.775043 & -3.499082 \\
\hline $\mathrm{H}$ & 1.932461 & 1.188978 & -4.317203 \\
\hline $\mathrm{C}$ & 1.110479 & -2.261118 & -0.788381 \\
\hline $\mathrm{C}$ & 1.921426 & -3.332420 & -0.358460 \\
\hline $\mathrm{C}$ & 0.345749 & -2.411194 & -1.9 \\
\hline $\mathrm{C}$ & 1.943161 & -4.534283 & -1.071226 \\
\hline $\mathrm{H}$ & 2.544250 & -3.225519 & 0.523287 \\
\hline $\mathrm{C}$ & 0.382584 & -3.612051 & -2.677025 \\
\hline $\mathrm{H}$ & -0.276570 & -1.601388 & -2.316448 \\
\hline $\mathrm{C}$ & 1.173343 & -4.676315 & -2.232317 \\
\hline $\mathrm{H}$ & 2.566844 & -5.352162 & -0.725991 \\
\hline $\mathrm{H}$ & -0.207376 & -3.710971 & -3.582037 \\
\hline $\mathrm{H}$ & 1.196558 & -5.607301 & -2.789262 \\
\hline $\mathrm{C}$ & 0.461813 & -0.970012 & 1.829831 \\
\hline $\mathrm{C}$ & 0.084794 & -2.260259 & 2.247294 \\
\hline $\mathrm{C}$ & 0.321918 & 0.113777 & 2.720167 \\
\hline $\mathrm{C}$ & -0.410594 & -2.464456 & 3.537792 \\
\hline $\mathrm{H}$ & 0.149383 & -3.098399 & 1.565290 \\
\hline $\mathrm{C}$ & -0.178808 & -0.101710 & 4.009364 \\
\hline $\mathrm{H}$ & 0.602459 & 1.116850 & 2.417817 \\
\hline $\mathrm{C}$ & -0.542732 & -1.387365 & 4.421987 \\
\hline $\mathrm{H}$ & -0.704289 & -3.462598 & 3.845610 \\
\hline $\mathrm{H}$ & -0.283326 & 0.741967 & 4.683089 \\
\hline $\mathrm{H}$ & -0.932350 & -1.548914 & 5.421855 \\
\hline
\end{tabular}




$\begin{array}{lrrr}\mathrm{C} & 2.926546 & -0.368376 & 0.325110 \\ \mathrm{C} & 3.782072 & -0.597838 & -0.771351 \\ \mathrm{C} & 3.484529 & 0.005949 & 1.560040 \\ \mathrm{C} & 5.162382 & -0.438419 & -0.635382 \\ \mathrm{H} & 3.374484 & -0.897571 & -1.727864 \\ \mathrm{C} & 4.868655 & 0.158152 & 1.691355 \\ \mathrm{H} & 2.852067 & 0.188169 & 2.416898 \\ \mathrm{C} & 5.709159 & -0.057937 & 0.596173 \\ \mathrm{H} & 5.808904 & -0.614525 & -1.488830 \\ \mathrm{H} & 5.282278 & 0.455275 & 2.648810 \\ \mathrm{H} & 6.782244 & 0.065816 & 0.700178\end{array}$


Table S19. Calculated cartesian coordinates and energy for complex 9cn.

\begin{tabular}{|c|c|c|c|}
\hline \multicolumn{4}{|c|}{ Energy $=-2902.80184125$} \\
\hline $\mathrm{Cl}$ & 0.798532 & 3.062124 & -2.261724 \\
\hline $\mathrm{Pd}$ & -0.668468 & 1.887921 & -0.879763 \\
\hline $\mathrm{Cl}$ & -1.938900 & 3.818143 & -0.418654 \\
\hline $\mathrm{C}$ & 0.220566 & -0.143782 & -0.947284 \\
\hline $\mathrm{C}$ & -1.189431 & -0.572065 & -0.713325 \\
\hline $\mathrm{N}$ & -1.887472 & 0.316279 & -0.079184 \\
\hline $\mathrm{P}$ & 1.391697 & -0.241329 & 0.462674 \\
\hline $\mathrm{C}$ & 0.862581 & -0.464808 & -2.242219 \\
\hline $\mathrm{O}$ & 2.067773 & -0.477306 & -2.432085 \\
\hline $\mathrm{O}$ & -0.048718 & -0.720071 & -3.202263 \\
\hline $\mathrm{C}$ & -1.744939 & -1.875888 & -1.263336 \\
\hline $\mathrm{O}$ & -1.222727 & -2.956166 & -1.064347 \\
\hline $\mathrm{O}$ & -2.843917 & -1.675567 & -1.984744 \\
\hline $\mathrm{C}$ & 0.499197 & -0.897842 & -4.543835 \\
\hline $\mathrm{C}$ & -3.461560 & -2.878126 & -2.540129 \\
\hline $\mathrm{H}$ & -4.298497 & -2.515523 & -3.133999 \\
\hline $\mathrm{H}$ & -3.808121 & -3.524787 & 1242 \\
\hline $\mathrm{H}$ & -2.742076 & -3.416704 & -3.159368 \\
\hline $\mathrm{H}$ & -0.364929 & -1.085799 & -5.179914 \\
\hline $\mathrm{H}$ & 1.193648 & -1.740184 & -4.562608 \\
\hline $\mathrm{H}$ & 1.015515 & 0.013170 & -4.850885 \\
\hline $\mathrm{C}$ & -3.197701 & & \\
\hline $\mathrm{C}$ & -3.537387 & -1.063918 & 707 \\
\hline $\mathrm{C}$ & -4.136442 & 1.165227 & 0.306136 \\
\hline $\mathrm{C}$ & -4.827646 & -1.227715 & 1.605093 \\
\hline $\mathrm{H}$ & -2.786502 & -1.831908 & 1.251054 \\
\hline $\mathrm{C}$ & -5.425854 & 0.982046 & \\
\hline $\mathrm{H}$ & -3.840178 & 2.094562 & -0.166553 \\
\hline $\mathrm{C}$ & -5.778618 & -0.210456 & 1.456195 \\
\hline $\mathrm{H}$ & -5.084647 & -2.143125 & 2.128617 \\
\hline $\mathrm{H}$ & -6.153180 & 1.780386 & 0.707658 \\
\hline $\mathrm{H}$ & -6.779624 & -0.339219 & 1.854990 \\
\hline $\mathrm{C}$ & 2.571841 & 1.134439 & 0.631893 \\
\hline $\mathrm{C}$ & 3.216393 & 1.684198 & -0.492962 \\
\hline $\mathrm{C}$ & 2.913632 & 1.594697 & 1.922439 \\
\hline $\mathrm{C}$ & 4.174097 & 2.688449 & -0.323019 \\
\hline $\mathrm{H}$ & 2.963867 & 1.342546 & -1.485642 \\
\hline $\mathrm{C}$ & 3.876545 & 2.594934 & 2.078567 \\
\hline $\mathrm{H}$ & 2.433724 & 1.181655 & 2.801063 \\
\hline $\mathrm{C}$ & 4.504766 & 3.146408 & 0.955768 \\
\hline $\mathrm{H}$ & 4.646106 & 3.120753 & -1.198041 \\
\hline $\mathrm{H}$ & 4.126924 & 2.945934 & 3.074165 \\
\hline $\mathrm{H}$ & 5.243852 & 3.931493 & 1.078456 \\
\hline $\mathrm{C}$ & 0.428668 & -0.310407 & 2.011524 \\
\hline $\mathrm{C}$ & 0.323161 & -1.511596 & 2.738011 \\
\hline $\mathrm{C}$ & -0.218235 & 0.848312 & 2.492574 \\
\hline $\mathrm{C}$ & -0.423933 & -1.554566 & 3.921036 \\
\hline $\mathrm{H}$ & 0.823977 & -2.407109 & 2.390689 \\
\hline $\mathrm{C}$ & -0.961544 & 0.795895 & 3.674203 \\
\hline $\mathrm{H}$ & -0.153682 & 1.781382 & 1.943521 \\
\hline $\mathrm{C}$ & -1.066674 & -0.403983 & 4.389140 \\
\hline $\mathrm{H}$ & -0.497047 & -2.485157 & 4.474034 \\
\hline $\mathrm{H}$ & -1.460835 & 1.690904 & 4.028463 \\
\hline $\mathrm{H}$ & -1.646239 & -0.439977 & 5.305824 \\
\hline
\end{tabular}


$\begin{array}{llll}\text { C } & 2.399093 & -1.768168 & 0.380879\end{array}$

$\begin{array}{llll}\text { C } & 3.752645 & -1.737180 & 0.767669\end{array}$

$\begin{array}{lllll}\text { C } & 1.828627 & -2.984171 & -0.042717\end{array}$

$\begin{array}{llll}\text { C } & 4.519287 & -2.906201 & 0.737837\end{array}$

$\begin{array}{llll}\mathrm{H} & 4.210994 & -0.806903 & 1.079723\end{array}$

$\begin{array}{llll}\text { C } & 2.603832 & -4.148026 & -0.068774\end{array}$

$\begin{array}{llll}\mathrm{H} & 0.791788 & -3.030600 & -0.357544\end{array}$

$\begin{array}{llll}\text { C } & 3.947793 & -4.112805 & 0.319970\end{array}$

$\begin{array}{llll}\mathrm{H} & 5.562538 & -2.869164 & 1.033427\end{array}$

$\begin{array}{llll}\mathrm{H} & 2.155712 & -5.078778 & -0.400854\end{array}$

$\begin{array}{llll}\mathrm{H} & 4.546593 & -5.017485 & 0.292343\end{array}$ 
Table S20. Comparison of Bond Distances ( $⿱$ ) $)$ and Angles (deg) Between L1, 3 and optimized L1 and 9 (B3LYP).

\begin{tabular}{|c|c|c|c|c|}
\hline Parameter & L1 & L1 (B3LYP) & 3 & $9($ B3LYP $)$ \\
\hline $\mathrm{P}-\mathrm{C} \alpha$ & $1.745(4)$ & 1.759 & $1.772(4)$ & 1.766 \\
\hline $\mathrm{P}-\mathrm{C}(\mathrm{Ph})$ & $1.805(4)$ & 1.837 & $1.796(3)$ & 1.824 \\
\hline $\mathrm{P}-\mathrm{C}(\mathrm{Ph})$ & $1.806(4)$ & 1.833 & $1.811(4)$ & 1.847 \\
\hline $\mathrm{P}-\mathrm{C}(\mathrm{Ph})$ & $1.807(4)$ & 1.833 & $1.812(4)$ & 1.852 \\
\hline $\mathrm{C} \alpha-\mathrm{C} \beta(\mathrm{N})$ & $1.442(5)$ & 1.446 & $1.413(5)$ & 1.447 \\
\hline $\mathrm{C} \alpha-\mathrm{C} \beta(\mathrm{O})$ & $1.424(5)$ & 1.451 & $1.443(5)$ & 1.441 \\
\hline$C \beta(N)-N$ & $1.287(4)$ & 1.298 & $1.306(4)$ & 1.310 \\
\hline $\mathrm{N}-\mathrm{C}(\mathrm{Ph})$ & $1.426(5)$ & 1.407 & $1.445(4)$ & 1.430 \\
\hline$C \beta(N)-C \gamma$ & $1.516(5)$ & 1.528 & $1.528(5)$ & 1.528 \\
\hline $\mathrm{C} \gamma=\mathrm{O}$ & $1.196(4)$ & 1.208 & $1.198(4)$ & 1.216 \\
\hline $\mathrm{C} \gamma-\mathrm{O}$ & $1.321(5)$ & 1.352 & $1.322(5)$ & 1.334 \\
\hline$C \beta(O)=O$ & $1.212(5)$ & 1.225 & $1.217(4)$ & 1.251 \\
\hline $\mathrm{C} \beta(\mathrm{O})-\mathrm{O}$ & $1.334(5)$ & 1.367 & $1.335(4)$ & 1.345 \\
\hline $\mathrm{P}-\mathrm{C} \alpha-\mathrm{C} \beta(\mathrm{N})$ & $113.6(3)$ & 114.9 & $126.2(3)$ & 124.0 \\
\hline $\mathrm{P}-\mathrm{C} \alpha-\mathrm{C} \beta(\mathrm{O})$ & $127.2(3)$ & 125.6 & $112.4(3)$ & 115.3 \\
\hline$C \beta(N)-C \alpha-C \beta(O)$ & $119.2(4)$ & 119.4 & $120.5(3)$ & 120.7 \\
\hline $\mathrm{C} \alpha-\mathrm{C} \beta(\mathrm{N})-\mathrm{N}$ & $119.8(4)$ & 119.8 & $128.0(3)$ & 120.9 \\
\hline $\mathrm{C} \alpha-\mathrm{C} \beta(\mathrm{N})-\mathrm{C} \gamma$ & $118.7(4)$ & 118.0 & $120.1(3)$ & 119.0 \\
\hline $\mathrm{N}-\mathrm{C} \beta(\mathrm{N})-\mathrm{C} \gamma$ & $121.5(4)$ & 122.0 & $112.0(3)$ & 119.6 \\
\hline$C \beta(N)-N-C(P h)$ & $122.0(4)$ & 124.2 & $119.2(3)$ & 121.6 \\
\hline $\mathrm{C} \alpha-\mathrm{C} \beta(\mathrm{O})=\mathrm{O}$ & $126.0(4)$ & 125.0 & $128.1(3)$ & 124.6 \\
\hline $\mathrm{C} \alpha-\mathrm{C} \beta(\mathrm{O})-\mathrm{O}$ & $114.6(4)$ & 113.8 & $113.9(3)$ & 114.0 \\
\hline $\mathrm{O}-\mathrm{C} \beta(\mathrm{O})=\mathrm{O}$ & $119.4(4)$ & 121.0 & $118.0(3)$ & 119.7 \\
\hline $\mathrm{P}-\mathrm{C} \alpha-\mathrm{C} \beta(\mathrm{N})-\mathrm{N}$ & $14.1(4)$ & -16.9 & -170.7 & 143.6 \\
\hline $\mathrm{P}-\mathrm{C} \alpha-\mathrm{C} \beta(\mathrm{O})-\mathrm{O}$ & -2.1 & 5.5 & 4.0 & 15.6 \\
\hline
\end{tabular}


Table S21. NPA Atomic Charges for Ylides L1 and CMPPY.

\begin{tabular}{|c|c|}
\hline & NPA atomic charge \\
\hline$q(P)$ on L1 & +0.86 \\
\hline$q(P)$ on CMPPY & +0.86 \\
\hline$q(C \alpha)$ on L1 & -0.69 \\
\hline$q(C \alpha)$ on CMPPY & -0.98 \\
\hline$q(\mathrm{O})$ on L1 & -0.63 \\
\hline$q(\mathrm{O})$ on CMPPY & -0.62 \\
\hline$q(N)$ on L1 & -0.57 \\
\hline$q\left(\mathrm{O}^{\prime}\right)$ on CMPPY & -0.63 \\
\hline
\end{tabular}

a) CMPPY: $\mathrm{H}_{3} \mathrm{P}=\mathrm{C}(\mathrm{H}) \mathrm{C}(\mathrm{O}) \mathrm{OMe}$, see Ref. 16 .

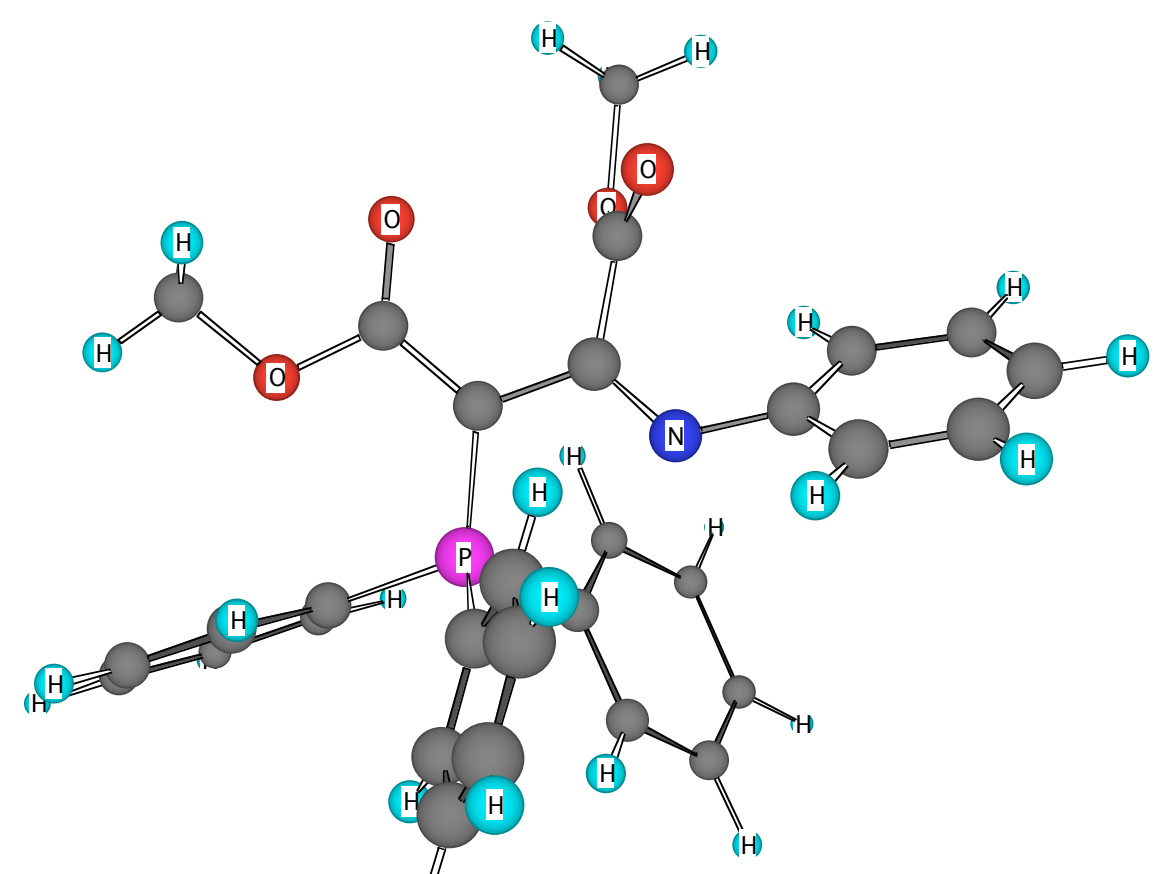

H

Figure S1. Optimized structure of ligand L1 

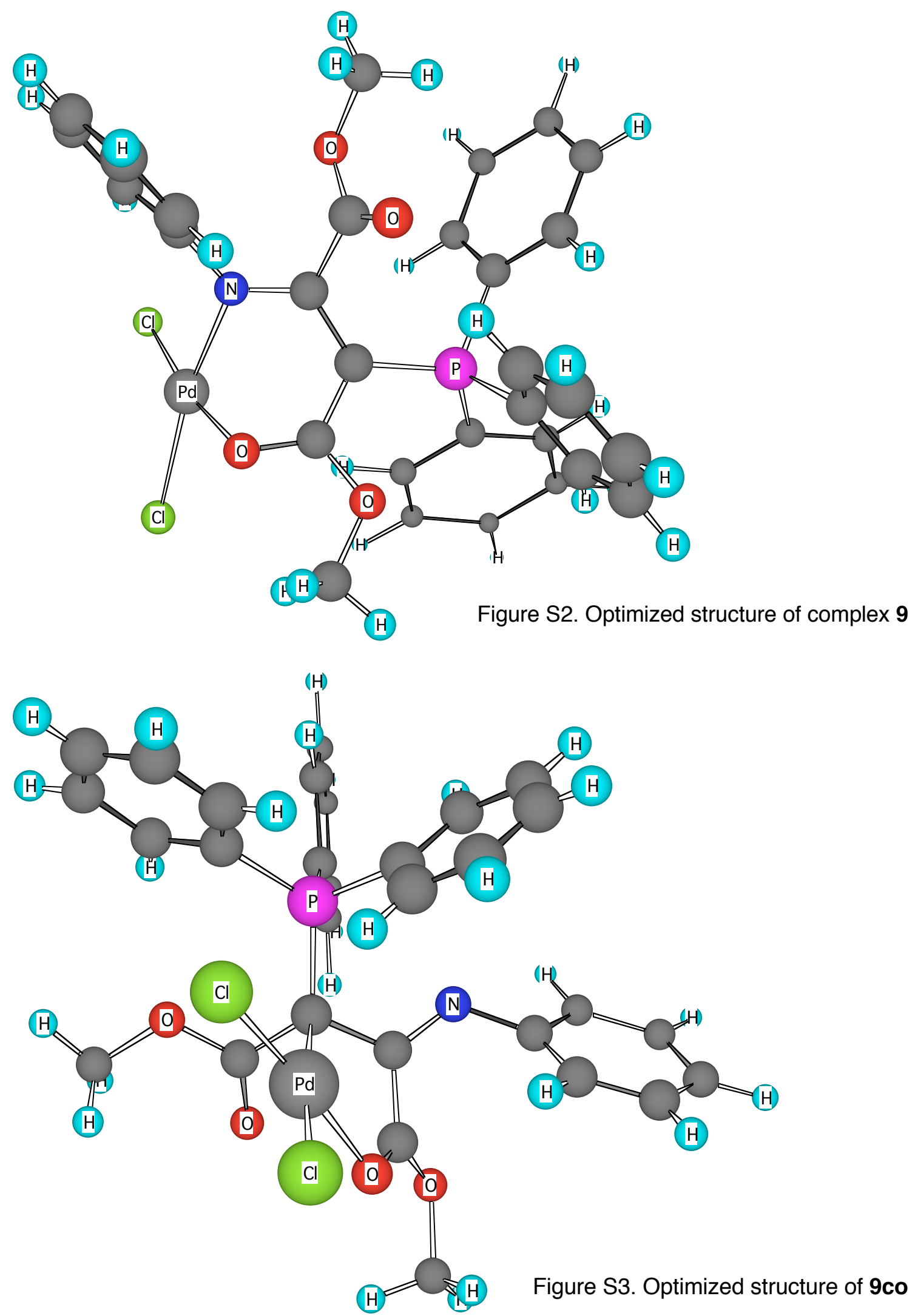


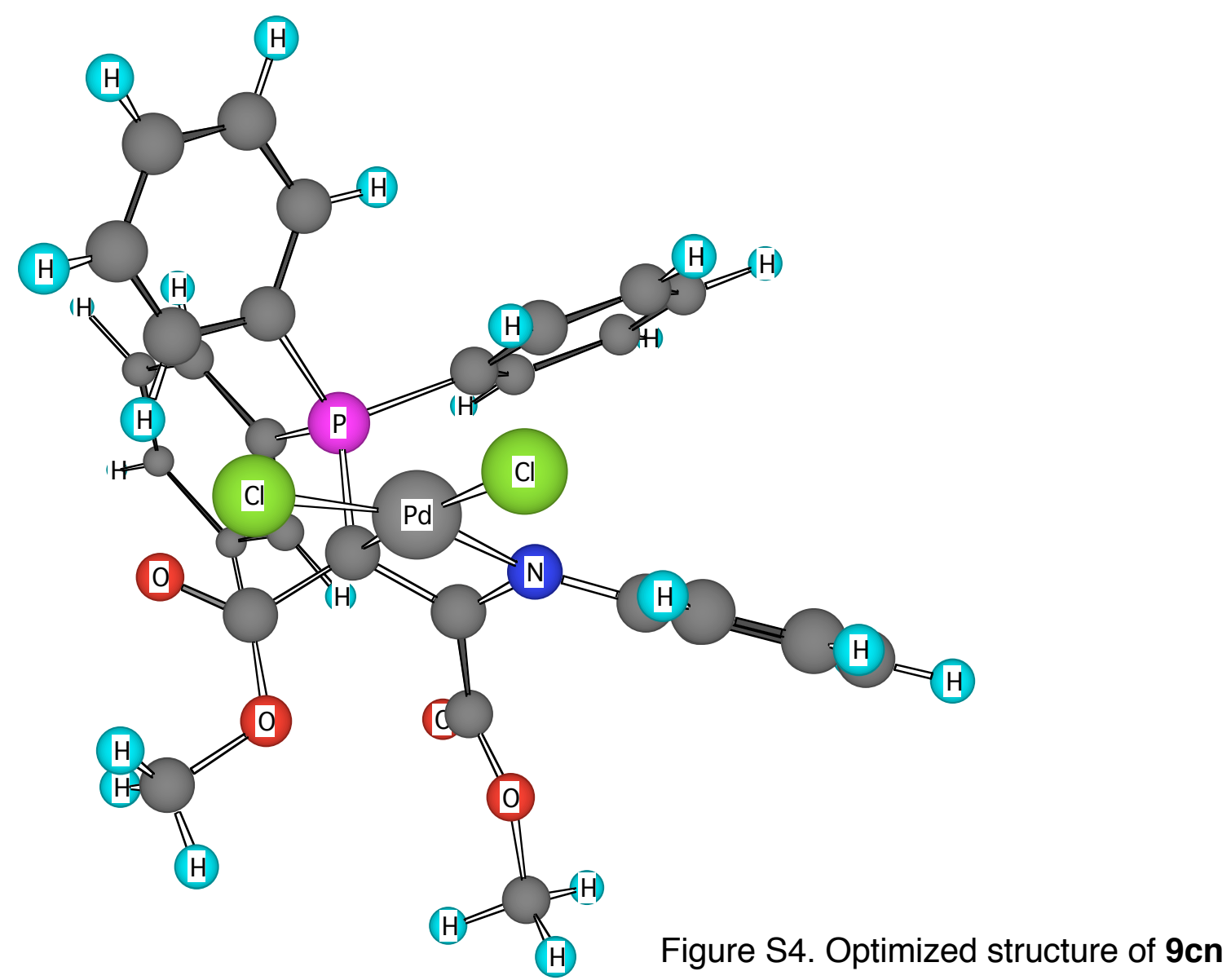

